\title{
Abstracts for the 42nd Human Genetics Society of Australasia Annual Scientific Meeting Sydney, New South Wales
} August 4-7, 2018

\section{Plenaries and Orals}

\section{Plenary 1 \\ LEVERAGING MASSIVE-SCALE DATABASES OF GENOMIC DATA TO IMPROVE INTERPRETATION OF GENETIC VARIANTS}

Daniel MacArthur

Broad Institute of MIT and Harvard, USA

More than two million humans have now had their exomes or genomes sequenced, but the underlying data are typically heavily siloed by project and generated using inconsistent sequencing and analysis technologies. Harmonizing these data represents a tremendous opportunity to understand the distribution of genetic variants across human genes, and their impact on human phenotypes and disease risk. In this talk I will describe work done as part of the Genome Aggregation Database (gnomAD) project and its predecessor, the Exome Aggregation Consortium (ExAC), to harmonize exome and genome sequencing data from over 130,000 individuals. I will describe the construction of this resource, and the ways it can be used to interpret individual genetic variants, to identify genes (and regions of genes) depleted of functional variation, and to characterize human 'knockouts' across a range of populations.

\section{Plenary 2 \\ GENOMICS AND THE BRAVE NEW WORLD OF PERSONALIZED MEDICINE: COLLABORATION ON A GLOBAL SCALE}

\section{Kathryn North AM}

Director, Murdoch Children's Research Institute, Australia

David Danks Professor of Child Health Research, University of Melbourne, Australia

Genomics is already having a huge impact on our ability to diagnose and understand of a range of disorders, and to target therapies to the individual. However, effective integration of this 'disruptive technology' into everyday clinical practice will require a 'whole-ofsystem' approach that builds on existing expertise. In Australia, we also need to overcome the 'state/federal divide' in the funding of genetic testing to develop a cohesive national approach that is cost effective and provides equitable access. Australian Genomics is an NHMRC-funded national collaborative network committed to im- plementing genomic medicine within Australia and providing evidence to inform policy and practice. Australian Genomics comprises over 80 partner organizations, including the diagnostic pathology and clinical genetics services of all Australian states and territories, along with the major research and academic institutions and peak professional bodies. Australian Genomics has four major work programs oriented around different challenges to integrating genomic medicine into Australian health care. These comprise a national diagnostic and research network; a federated data infrastructure; a focus on regulatory, economic policy and examination of the barriers to implementation; and an education, ethics and workforce focus. Our clinical programs are currently piloting genomic medicine for patients with rare diseases or cancers across multiple flagships. Each flagship project is examining the clinical utility of a variety of genomic sequencing technologies and using the resulting data to support data sharing and inform the regulatory, ethical, economic, policy and workforce infrastructure required to integrate genomics as a key part of the Australian health system. Australian Genomics is also a leading member and driver project of the Global Alliance for Genomics and Health, an organization of over 500 of the world's leading biomedical research institutions, healthcare providers, information technology and life science companies, funders of research, and disease and patient advocacy organizations. The Global Alliance aims to accelerate the world-wide effort to responsibly aggregate, analyze and share large amounts of genomic and clinical information to advance the understanding, diagnosis, and treatment for cancer, inherited diseases, infectious diseases, and drug responses.

\section{Plenary 3}

THE GENOMIC JOURNEY: MAPPING THE PATHWAY TOWARDS ROUTINE ACCESS TO GENOMIC INFORMATION TO INFORM HEALTHCARE

\footnotetext{
Marcel E. Dinger ${ }^{1,2,3}$

${ }^{l}$ Kinghorn Centre for Clinical Genomics, Garvan Institute for Medical Research, Sydney, Australia

2 St Vincents Clinical School, UNSW Sydney, Sydney, Australia

${ }^{3}$ Genome.One, Sydney, Australia
}

Genomic information has the potential to improve management or potentially prevent or enable early intervention of virtually 
every disease. To do so, genomic information needs to be routinely accessible and easily interpretable in any clinical interaction where genetics may inform treatment decisions and patient management. The realization of this future will necessitate a shift away from the current paradigm of genetic testing, which follows a traditional model of a transactional relationship between a specialist referrer and a pathology laboratory, to one where genome interpretation is facilitated digitally at point-of-care. The digital transformation of genetic testing will require the gradual and systematic overcoming of numerous obstacles; technological, cultural and political. In this presentation, I will highlight local and global trends in how this transformation is starting to occur and where the key challenges are likely to lie in the future.

\section{Plenary 4 \\ NEW GENE-BASED TREATMENTS FOR METHYLMALONIC ACIDEMIA}

\section{Charles Venditti}

National Human Genome Research Institute; Organic Acid Research Section, National Institutes of Health, Bethesda, USA

Hereditary methylmalonic acidemia (MMA) caused by deficiency of the enzyme methylmalonyl-coA mutase (MUT) is a relatively common and severe organic acidemia. The recalcitrant nature of the condition to conventional dietary and medical management has led to the use of elective liver and combined liver-kidney transplantation in some patients. In this talk, I will review preclinical studies that present alternative approaches to transplantation as a treatment for MMA, including mRNA and AAV gene therapy.

\section{Plenary 5}

TREATING SPINAL MUSCULAR ATROPHY

\section{Farrar MA}

Sydney Children's Hospital, Sydney, NSW, Australia

With the first approved therapy, Spinraza, spinal muscular atrophy (SMA) is now a treatable neurodegenerative disease. This longawaited first step may provide meaningful benefits to patients and their families, along with new challenges and opportunities for clinicians and researchers. This presentation will discuss the development and clinical translation of therapies for SMA, beginning with reviewing the genetic underpinnings. The mechanisms of action of new technologies, such as molecular and gene replacement therapy, and major findings of clinical trials will be discussed, along with the various benefits and limitations. With the reality of novel therapies, there are challenges defining standards of care as the natural history evolves and treatment paradigms transform. Management may now include active therapy in addition to multidisciplinary supportive care. Prognosis and life-expectancy are now uncertain in patients treated with novel therapies. New challenges arise including difficulties with access to, cost of, and resources for treatment administration. In addition, there are challenges for early diagnosis, prompting efforts to undertake newborn screening for SMA. Furthermore, reproductive carrier screening is important in providing a holistic approach. There is a need for continued research to address many remaining uncertainties - what are the limits of therapy, utility of combination therapy, the long-term impact and safety, and the feasibility, utility and acceptability of screening? Progress thus far in treating SMA shows the potential of new technologies to transform medicine.

\section{Plenary 6 \\ TRANSLATING GENES INTO MEDICINES}

\author{
John EJ Rasko 1,2,3 \\ ${ }^{1}$ Cell \& Molecular Therapies, Royal Prince Alfred Hospital, Sydney, Australia \\ ${ }^{2}$ Gene and Stem Cell Therapy Program, Centenary Institute, Sydney, Australia \\ ${ }^{3}$ Sydney Medical School, University of Sydney, Australia
}

The field of genetics originally provided insights into disease pathogenesis. More recently, genomic medicine has facilitated improved prognostication, reproductive choices and therapeutic drug options. The challenge of realizing the full potential of genetic understanding has been in overcoming the hurdles of efficient gene therapy. Since the first human clinical trial using gene technology in 1989, there have been over 2,400 approved clinical trials worldwide. A few dozen clinical trials have been undertaken in Australia representing just over $1 \%$ of the trials worldwide. The overwhelming majority of human clinical trials involve short-term gene expression or random integration of a therapeutic gene. Emerging technologies require controlled development in compliance with safety, regulatory and GMP requirements. The pace of gene therapy clinical development has accelerated dramatically over recent years. The convergence of substantial incremental technical advances towards combined cell and gene therapy has led to improved clinical outcomes in immune deficiencies, hemoglobinopathies, immunotherapies and other inherited diseases. Highlights in the clinical gene therapy field will be discussed with special reference to hemophilia, thalassemia, blindness and cancer.

\section{Plenary 7 \\ AN EVIDENCE-BASED FRAMEWORK FOR EVALUATING THE CLINICAL VALIDITY OF GENE-DISEASE ASSOCIATIONS \\ Steven M. Harrison' \\ ${ }^{I}$ Partners HealthCare Laboratory for Molecular Medicine and The Broad Institute of MIT and Harvard, Cambridge, MA, USA}

With advances in genomic sequencing technology, the number of reported gene-disease relationships has rapidly expanded. However, the evidence supporting these claims varies widely, confounding accurate evaluation of genomic variation in a clinical setting. Understanding the validity of gene-disease associations is necessary as returning genetic testing results in genes with an unclear role in disease could lead to incorrect diagnoses and/or errant management of the affected individual and their families. To help differentiate clinically valid relationships from less well-substantiated relationships, the NIH-funded Clinical Genome Resource (ClinGen) has developed a framework to define and evaluate the clinical validity of gene-disease pairs across a variety of Mendelian disorders (Strande et al, 2017; PMID:28552198). This framework includes evaluation of relevant genetic and experimental evidence supporting or contradicting a gene-disease relationship. This semi-quantitative measurement for the strength of evidence of a gene-disease relationship then correlates to a qualitative classification: 'Definitive,' 'Strong,' 'Moderate,' 'Limited,' 'No Reported Evidence,' or 'Conflicting Evidence.' Nearly 500 gene-disease associations have been completed by ClinGen working groups and the results are shared on the ClinGen website (https://search.clinicalgenome.org/ $\mathrm{kb} /$ gene-validity). This evidence-based, systematic method to assess the strength of gene-disease relationships will facilitate more knowledgeable utilization of genomic variants in clinical and research settings. 


\section{Plenary 8}

THE IMPACT AND IMPLICATIONS OF

LANGUAGE-EFFECTIVE COMMUNICATION STRATEGIES FOR BETTER HEALTH OUTCOMES

Rachel Callander BFA FNZIPP

I work with health professionals across many specialties, teaching them empowering and effective communication solutions to use with patients or parents. Studies prove that the language used at diagnosis, or when delivering life changing medical information, is vitally important and so often, used with negative outcomes. I teach that using effective communication, is just as important as any medical therapy or procedure, and that the difference between a powerless and empowered patient or parent, is determined by language. This learning is being used to great effect across many different areas of health care, and changes are being made which has huge impact on positive patient outcomes and practitioner wellbeing.

\section{Plenary 9 \\ GENETIC CARRIER SCREENING IN AUSTRALASIA: RESEARCH AND CLINICAL PRACTICE - WHAT HAVE WE LEARNED AND WHERE TO FROM HERE?}

Alison Dalton Archibald

Victorian Clinical Genetics Services (VCGS), Melbourne, VIC, Australia

Advances in genetic testing technology have increased our capacity to identify prospective parents at risk of having a child with an inherited condition. Carrier screening has shifted from testing for a single or a small number of conditions, to much larger panels. However, despite its availability, the majority of people are not offered genetic carrier screening pre-conception or in early pregnancy. Guidelines are beginning to acknowledge the availability of carrier screening panels and now suggest carrier screening can be offered regardless of family history. Australian research demonstrates support for carrier screening from families impacted by these conditions, healthcare providers, those offered screening, and the general community. However, the key challenge is how to optimally deliver genetic carrier screening services. In 2012, Victorian Clinical Genetics Services launched the prepair ${ }^{\mathrm{TM}}$ test, screening for carriers of cystic fibrosis, fragile $\mathrm{X}$ syndrome and spinal muscular atrophy. Our experience of the first 20,000 individuals screened along with recent research highlights that accessibility and appropriately managing carrier results are key considerations. To facilitate informed-decisionmaking and to assist health professionals in offering screening, creative approaches to information delivery are needed. Careful design of screening panels will be essential in ensuring useful information for reproductive decision-making is provided and the chance of ambiguous or uncertain results is minimized. Coordination of carrier screening by clinical genetics services in partnership with laboratory teams, specialist physicians and community support organizations is important in making information, support, education and genetic counseling available to those offering, considering or undergoing screening.

\section{Plenary 10 \\ NEWBORN BLOODSPOT SCREENING: A NATIONAL POLICY FRAMEWORK}

\section{Bridget Wilcken}

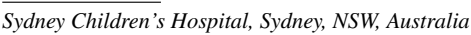

Population-wide dried blood-spot newborn screening started over 50 years ago, first in the USA, and quickly in other countries with well-developed health systems, including Australasia. The initial screening was for phenylketonuria, soon followed by a few other disorders, including congenital hypothyroidism, galactosemia, congenital adrenal hyperplasia and, less widely, cystic fibrosis. Tandem mass-spectrometry allowed a newborn screening revolution in the late 90 's when a test could be multiplexed — some 28 aminoand organic-acid marker compounds allowed simultaneous screening for over 40 disorders. This greatly expanded newborn screening, plus the development of new forms of treatment, led to demand for addition of many disorders to screening programs, and also to recognition that for evidence-based screening national policies were needed, consistent with current aims. Processes for decision making about adding disorders were developed in many jurisdictions, including the United Kingdom (decision making established in 2009), New Zealand (2011) the United States (finalized in 2013). Australia has trailed the international community. Newborn screening was exclusively state-based, with five separate programs, three smaller jurisdictions outsourcing to larger ones. The Human Genetics Society has been the only forum for collaboration and non-binding decision making. Now, after several years' work, a National Policy Framework has been released. This outlines aims and objectives of screening, implementation, quality and safety, monitoring, evaluation, and formal decision-making processes. Several significant disorders, screened for elsewhere, are waiting in the wings for assessment. Australia can benefit from international experience, both to include, and not to include, new disorders. Exciting times for newborn screening.

\section{Plenary 11 \\ POPULATION SCREENING - PAST, PRESENT AND FUTURE}

Anne E Cust $^{1,2}$

${ }^{I}$ Cancer Epidemiology and Prevention Research, Sydney School of Public Health,

Faculty of Medicine and Health, The University of Sydney, Australia

${ }^{2}$ Melanoma Institute Australia, The University of Sydney, Australia

Most cancer screening programs have been designed for the 'average person or patient'. But this 'one-size-fits-all-approach' is changing with the emergence of precision medicine, an innovative approach to disease prevention, screening and treatment that takes into account individuals' differences in genes, environments, and lifestyles. Precision medicine approaches could play a major role in cancer screening. Current cancer screening programs for asymptomatic Australians are based on age, but advances in genomics and risk prediction modelling allow a more personalized, risk-stratified screening approach that is potentially more effective and efficient. By stratifying the population into different risk groups according to genomic risk alongside traditional risk factors (such as age, family history, lifestyle factors), screening could be tailored to each risk group, for example, with different start and end ages, screening intervals and modalities. More precise cancer risk assessment, coupled with personalization of screening regimens based on risk, could lead to programs that derive maximal benefit of screening for subsets of the population at higher cancer risk, while resulting in less screening and thus less potential harm (e.g., psychological burden, overdiagnosis, overtreatment) for those at lower risk than do current screening programs based on age criteria alone. This talk will discuss translation of genomics into precision cancer screening programs from a multidisciplinary perspective, including the experience of the Melanoma Genomics Managing Your Risk Study. 


\section{Sutherland Lecture THE VISUAL GENOME BRIDGING TO NEW PROSPECTS FOR SIGHT}

\author{
Robyn Jamieson ${ }^{1,2,3}$ \\ ${ }^{1}$ Discipline of Genomic Medicine, University of Sydney, Sydney, NSW, Australia \\ ${ }^{2}$ Western Sydney Genetics Program, The Children's Hospital at Westmead, Sydney \\ Children's Hospitals Network, Sydney, NSW, Australia \\ ${ }^{3}$ Eye Genetics Research Unit, Children's Medical Research Institute, University of \\ Sydney, Sydney, NSW, Australia
}

Genomics is a powerful tool for molecular diagnosis and prospects for vision in blinding genetic eye diseases. Genetically heterogeneous developmental eye diseases and retinal degenerative conditions have mutation detection rates in the vicinity of 50-75\% using genomic methods. Frontiers for further investigation include determination of the functional significance of deeply intronic splicing and other variants of uncertain significance, novel disease gene identification, and advancement of therapeutic strategies. Differentiation of human induced pluripotent stem cells to retinal organoids and retinal pigment epithelial cells implements an effective model system for interrogation of the functional significance of variants. Retinal organoids and pigment epithelial cells also give a useful platform to test novel gene replacement, and gene or base editing strategies. The combination of cellular, organoid and animal model approaches provides a powerful resource for functional investigation and testing of novel therapies and vector delivery systems for application to the retinal dystrophies. With early indicators of success in gene therapy clinical trials for some of the retinal dystrophies, functional and therapeutic genomic applications hold great promise for beneficial impact for patients with currently untreatable blinding eye conditions.

\section{Plenary 13 \\ HOW TO AVOID DROWNING WHEN DRINKING FROM THE FIREHOSE: A DECISION AID EMPOWERING PATIENTS IN THE ERA OF GENOME SEQUENCING}

\author{
Yvonne Bombard ${ }^{1,2}$, Marc Clausen $^{2}$, Salma Shickh ${ }^{2}$, Chloe Mighton $^{2}$, Selina \\ Casalino $^{2,10}$, Emily Glogowski ${ }^{3}$, Kasmintan Schrader ${ }^{4}$, Adena Scheer ${ }^{1,2}$, Jordan \\ Lerner-Ellis ${ }^{1,8}$, Christine Elser ${ }^{6}$, Seema Panchal ${ }^{1,8}$, Andrea Eisen ${ }^{7}$, Tracy Graham ${ }^{7}$, \\ Melyssa Aronson 1,9, Laura Winter-Paquette ${ }^{1,9}$, Kara Semotiuk 1,9, Michael \\ Evans $^{1,2}$, June Carroll ${ }^{8}$, Jada Hamilton ${ }^{5}$, Kenneth Offit ${ }^{5}$, Mark Robson ${ }^{5}$, Kevin \\ Thorpe ${ }^{1}$, Andreas Laupacis ${ }^{1,2}$ \\ ${ }^{1}$ University of Toronto, Toronto, Ontario, Canada \\ 2 St. Michael's Hospital, Toronto, Ontario, Canada \\ GeneDx, Gaithersburg, Maryland, USA \\ ${ }^{4}$ BC Cancer Agency, Vancouver, BC, Canada \\ ${ }^{5}$ Memorial Sloan Kettering Cancer Center, New York, USA \\ ${ }^{6}$ University Health Network, Toronto, Ontario, Canada \\ ${ }^{7}$ Sunnybrook Health Sciences Centre, Toronto, Ontario, Canada \\ ${ }^{8}$ Mount Sinai Hospital, Sinai Health System, Toronto, Ontario, Canada \\ ${ }^{9}$ Zane Cohen Centre for Digestive Diseases, Sinai Health System, Toronto, Ontario, \\ Canada \\ ${ }^{10}$ McMaster University, Hamilton, Ontario, Canada
}

Background: The volume and complexity of incidental results (IR) from genome sequencing (GS) make engaging in pretest shared decision making with patients infeasible. To address this care gap, we created an interactive, online decision aid (DA) (www. genomicsadviser.com) to guide patients' selection of IR. Methods: We conducted a superiority RCT of the DA among adult patients who received uninformative results from past genetic testing for colorectal or breast cancer, for whom GS may be informative as a second-tier test. Intervention arm patients used the DA to make a hypothetical selection of IR, then spoke briefly with a genetic counselor (GC). Control arm patients spoke with a GC to select IR. The primary outcome was decisional conflict. Secondary outcomes were knowledge, preparation for decision-making, and satisfaction with decision. We conducted semi-structured interviews with a subset of patients $(n=31)$ to characterize their decision-making process for IR. Results: We enrolled 133 patients (90\% female; $60 \% \geq 50$ yo).
No statistically significant differences in decisional conflict, knowledge of sequencing limitations, preparation for decision making, satisfaction with decision were found between arms. Intervention arm participants had significantly higher scores on the knowledge benefits subscale $(p=.01)$. Thematic analysis identified characteristics that informed participants' decisions regarding IR: attitude toward IR, concerns, perceived utility, self-definition, and contextual factors. Based on how these characteristics were shared among groups of participants, we identified five patient profiles: Information Enthusiasts, Concerned, Contemplators, Advanced Life Stage, and Reassurance Seekers, which will be described in depth. Discussion: Patient profiles could assist providers tailor their counseling to patients' decisional needs. The Genomics ADvISER is not superior to genetic counseling but could serve as an educational tool for IR, reducing in-clinic education time and potentially health care costs.

\section{Plenary 14 \\ THE GENOMIC AUTOPSY STUDY: USING GENOMICS AS AN ADJUNCT TO STANDARD AUTOPSY TO UNLOCK THE CAUSE OF COMPLEX FETAL AND NEONATAL \\ PRESENTATIONS}

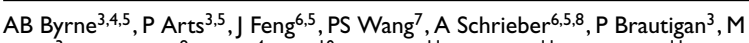
Babic $^{3}$, W Waters 9 , L Pais ${ }^{4}$, S Yu ${ }^{10}$, J Lipsett ${ }^{11}$, L Moore ${ }^{11}$, N Manton ${ }^{11}, Y$

Khong ' ', E Luddington' ', E Thompson', J Liebelt' ', L McGregor', M Dinger '2, DG MacArthur ${ }^{4}$, S King-Smith ${ }^{13,14}, \mathrm{C} \mathrm{Hahn}{ }^{13,15,5}, \mathrm{~K}$ Kassahn $^{3,8}$, ${ }^{*} \mathrm{H} \mathrm{Scott}^{3,16,17,15}$; *CP Barnett ${ }^{1,2}$ (*co-senior authors)

${ }^{1}$ Paediatric and Reproductive Genetics Unit, Women's and Children's Hospital, North Adelaide, Australia

${ }^{2}$ University of Adelaide, Adelaide, Australia

${ }^{3}$ Genetics and Molecular Pathology Research Laboratory, Centre for Cancer Biology, an alliance between SA Pathology and the University of South Australia, Adelaide, Australia

${ }^{4}$ Center for Mendelian Genomics, Broad Institute of MIT and Harvard, Cambridge, MA, United States

${ }_{5}^{5}$ School of Pharmacy and Medical Sciences, University of South Australia, Adelaide, Australia

${ }^{6}$ ACRF Cancer Genomics Facility, Centre for Cancer Biology, an alliance between SA ${ }^{6}$ ACRF Cancer Genomics Facility, Centre for Cancer Biology, an allian
Pathology and the University of South Australia, Adelaide, Australia

${ }^{7}$ 3ACRF Cancer Genomics Facility, Centre for Cancer Biology, an alliance between

SA Pathology and the University of South Australia, Adelaide, Australia

${ }^{8}$ School of Biological Sciences, University of Adelaide, Adelaide, Australia

${ }_{9}^{4}$ 4Department of Genetics and Molecular Pathology, SA Pathology, North Adelaide, Australia

${ }^{10}$ Department of Genetics and Molecular Pathology, SA Pathology, North Adelaide, Australia

${ }^{11}$ Department of Anatomical Pathology, SA Pathology at Women's and Children's

Hospital, North Adelaide, Australia

12 Kinghorn Centre for Clinical Genomics, Garvan Institute of Medical Research,

Sydney, Australia

${ }^{13}$ Genetics and Molecular Pathology Research Laboratory, Centre for Cancer Biology, an alliance between SA Pathology and the University of South Australia, North

Adelaide, Australia

${ }_{14}^{14}$ Australian Genomic Health Alliance, Melbourne, Australia

${ }^{15}$ School of Medicine, University of Adelaide, Adelaide, Australia

${ }^{16}$ ACRF Cancer Genomics Facility, Centre for Cancer Biology, an alliance between SA Pathology and the University of South Australia, Adelaide, Australia

${ }^{17}$ Department of Genetics and Molecular Pathology, SA Pathology, Adelaide, Australia

Background: The cause of pregnancy loss and perinatal death remains unexplained in at least $25 \%$ of cases, despite a high perinatal autopsy rate in Australia. The most common factor contributing to perinatal death is congenital abnormalities. Aim: To use WES and WGS to identify genetic causes of fetal/newborn abnormalities that result in termination of pregnancy, death in utero or in the newborn period, in view to providing families with answers regarding cause and likelihood of recurrence. Methods: WES and/or WGS is being performed using Illumina sequencing systems, if microarray has not identified a cause. Prospective cases are families referred to the Genetics unit (parent-fetus trios) and retrospective cases are from stored autopsy samples (singletons). High priority cases are consanguineous families, fetuses with multiple malformations, and unexplained fetal/newborn death. Statistical, bioinformatic and experimental laboratory techniques are used to confirm causality of variants. Results: 43 prospective trios and 60 retrospective singletons have been recruited and sequenced. Of the prospective cohort, 
$23 \%$ of cases have been solved and an additional $26 \%$ have a single promising candidate being investigated. In the retrospective cohort, $18 \%$ of cases have strong candidates identified. Solved cases include new disease gene discoveries, new syndrome identification and novel severe fetal presentations of existing rare pediatric disease. The study has contributed directly to the birth of 3 healthy newborns. Discussion: Our results provide insights into molecular mechanisms of early development and indicate that a genomic autopsy using WES/WGS should be a routine component of the investigation of pregnancy loss and perinatal death.

\section{Plenary 15}

\section{THE POWER OF MYCODE: SOMEDAY IS TODAY}

\section{Amy Curry Sturm \\ Geisinger, Genomic Medicine Institute, Danville, Pennsylvania, USA}

The MyCode Community Health Initiative is a precision medicine project that began at Geisinger, a large integrated healthcare system in Pennsylvania, US, in 2007. It includes a system-wide biobank linked to the electronic health record for research use by Geisinger and Geisinger collaborators. Currently, there are over 195,000 enrolled. In 2014, Geisinger established the 'DiscovEHR' collaboration with Regeneron Genetics Center to sequence more than 250,000 patient-participants and currently over 92,000 have undergone whole exome sequencing. Over time, the decision was made to return actionable results to patient-participants, with the first result being returned in May 2015. Initial data published in Science shows that approximately $3.5 \%$ of individuals in the study have a 'clinically actionable' variant in one of the genes on the initially developed Geisinger gene list. Today, the MyCode Genomic Screening and Counseling Program has returned over 550 results, including those that confer risk for hereditary cardiovascular and cancer conditions. An infrastructure and process to return results to patientparticipants and their healthcare providers has been established and undergoes continual process improvements under the auspices of Geisinger's learning healthcare system. We have developed and are testing scalable approaches for consent, patient follow-up, and family communication and cascade testing. Early results have shown the potential clinical impact of this program, with patient-participants being diagnosed with early-stage cancers and types of heart disease that have required medical interventions. The history, process, and patient examples will be shared during this presentation.

\section{HGSA Oration \\ I WANTED TO BE A GENETIC COUNSELOR ... AND THIS IS MY STORY}

\section{Kristine Barlow-Stewart}

University of Sydney, Sydney, NSW, Australia

While this is the 42nd Annual Scientific Meeting, the first HGSA Oration was not until 1990, given by Dr Tony Pollard, at the Perth meeting. 1990 was also the year that I submitted my portfolio for Certification in Genetic Counselling, awarded in 1991. The road to that point was full of twists, turns and potholes and required several detours. The journey began with my commitment in the early 70's to becoming a genetic counselor like those in the USA and thinking that undertaking a $\mathrm{PhD}$ in genetics would open the door; to my first appointment in 1981 as an Honorary Genetics Associate at the Royal Alexandra Children's Hospital in Sydney; to working with the HGSA to establish a Board of Censors in Genetic Counselling from 1986 and developing the Guidelines for Certification. Along the way I learnt of the importance of genetics education underpinning the practice of genetic counseling and so led to the establishment of the Centre for Genetics Education in 1989. I also listened to families dealing with genetic conditions: their needs for information and support and contact with other families experiencing the same challenges. That led to the establishment of AGSA in 1989. However, I have not travelled this road alone. This Oration gives me the chance to honor and acknowledge those who have provided mentoring and support to me as well as the profession along this journey and beyond to the point where genetic counseling is recognized as a critically important discipline in the genomic era.

\section{Plenary 16 \\ THE CHANGING FACE OF SYNDROME DIAGNOSIS: TRANSLATING RESEARCH INTO FUTURE PRACTICE}

\author{
Jill Clayton-Smith \\ Manchester Centre For Genomic Medicine, Manchester University Hospitals, \\ Manchester, UK \\ Division of Evolution and Genomic Sciences, University of Manchester, UK
}

Syndrome diagnosis began as a pediatric specialty practiced by clinicians with keen observational skills and an aptitude for recognising patterns of malformations, development and behavior. The ability to delineate syndromes and ascertain cohorts of similarly affected individuals for genetic research facilitated identification of the genetic basis of many disorders. The majority of patients remained undiagnosed, however, so newer technologies including array CGH and Next Generation sequencing have been welcome additions to the diagnostic armamentarium, enabling identification of the genetic basis of rarer disorders and those with less specific clinical features. Some important lessons have been learned, for example about second phenotypes in known genes and the frequency of blended phenotypes. We have, however, faced the challenges of interpretation of variants in little studied genes, the limitations of most bioinformatic pipelines, and communication of complex genomic information. Importantly, many syndromic disorders have remained undiagnosed after whole genome sequencing and although reanalysis of data solves some cases, other approaches will be needed to solve others. This presentation will use case examples to demonstrate some lessons we have learned from large scale sequencing studies, review those mechanisms which will require alternative approaches and discuss applications such as face-recognition technology and computational phenomics. The skills needed to be an expert in syndrome diagnosis in the current era differ from those of the first dysmorphologists but the increased scope for reverse phenotyping and identifying possibilities for treatments together with an import role in multidisciplinary teams suggest that this is likely to remain an important discipline.

\section{Concurrent Session - Submitted Orals \\ Oral 1 \\ A NEW FRAMEWORK FOR PRIORITIZATION OF DISEASE-CAUSING RARE VARIANTS IN DILATED CARDIOMYOPATHY}

Horvat $C^{\prime}$, Mazzarotto $F^{2,3}$, Lam L ${ }^{4,5}$, Munro J', Johnson R', Giannoulatou E',

Seidman $\mathrm{J}^{4,5}$, Cook $\mathrm{S}^{2,3,6,7}$, Seidman $\mathrm{C}^{4,8}$, Fatkin $\mathrm{D}^{1,9,10}$

${ }^{1}$ Victor Chang Cardiac Research Institute, Sydney, Australia

${ }^{2}$ NIHR Cardiovascular Biomedical Research Unit, London, UK

${ }^{3}$ Imperial College London, London, UK

${ }^{4}$ Department of Genetics, Harvard Medical School, Boston, USA

${ }^{5}$ Howard Hughes Medical Institute, Boston, USA

${ }^{6}$ Duke-National University of Singapore, Singapore

${ }^{7}$ National Heart Center Singapore, Singapore

${ }^{8}$ Cardiovascular Division, Brigham and Women's Hospital, Boston, USA

${ }^{9}$ Cardiology Department, St Vincent's Hospital, Sydney, Australia

${ }^{10}$ Faculty of Medicine, University of New South Wales, Sydney, Australia

With the rise of next generation sequencing, the discovery of rare variants within disease associated genes in the general population is increasing. Consequently, classifying variants found through 
cardiac genetic testing and identifying those that are truly diseasecausing becomes a challenge. Here we aim to develop robust criteria to differentiate rare variants in patients with dilated cardiomyopathy (DCM) and healthy control subjects. Rare (minor allele frequency $<0.1 \%$ ) variants in 41 cardiomyopathy-associated genes were identified using targeted re-sequencing panels in 532 DCM patients and 527 healthy control subjects. Variants were further assessed for pathogenicity using a combination of variant-based and gene-based parameters. Protein-altering rare variants were common in both DCM $(77 \%)$ and control $(66 \%)$ cohorts. While truncating TTN variants were present in $16 \%$ of probands and only $1 \%$ of controls, the prevalence of truncating variants in other genes was relatively low (3\%) and not significantly different between cases and controls. Inferring pathogenicity based on absence from population databases, and predicted to be functionally deleterious in silico, provided some discriminative value for missense variants between the DCM and control subjects. However inclusion of prioritization of genes according to their relative importance in DCM pathogenesis led to a much better distinction of variant pathogenesis between cohorts, with odds of DCM nearly 9-fold for truncating or high-impact missense variants in genes with the strongest evidence of DCM causation $(p<.0001)$. By utilising both variant- and gene-level information, rare variants in cardiomyopathy genes can be effectively stratified to identify those with clinical relevance.

\section{Oral 2 \\ MUTATIONS IN THE EPITHELIAL CADHERIN-P120-CATENIN COMPLEX CAUSE MENDELIAN NON-SYNDROMIC CLEFT LIP AND PALATE}

Roscioli $T^{18,29,30}$, Cox $L^{1,2,3}$, Cox $T^{1,2,4}$, Moreno Uribe $L^{5}$, Zhu $Y^{6,7}$, Richter $C^{5}$, Nidey $N^{8}$, Standley $]^{8}$, Deng $M^{9}$, Blue $E^{10}$, Chong $J^{11}$, Yang $Y^{12}$, Carstens $R^{12,13}$, Anand $D^{14}$, Lachke $S^{14}$, Smith $J^{15}$, Dorschner $M^{16,17}$, Bedell $B^{8}$, Kirk $E^{6,18}$, Hing $\mathrm{A}^{1,19}$, Venselaar $\mathrm{H}^{20}$, Valencia Ramirez $\mathrm{L}^{21}$, Bamshad $\mathrm{M}^{11,15}$, Glass $\mathrm{I}^{9,11}$, Cooper $\mathrm{J}^{3}$, Haan $E^{22,23}$, Nickerson $D^{15}$, van Bokhoven $\mathrm{H}^{24,25}$, Zhou $\mathrm{H}^{24,26}$, Nash Krahn $\mathrm{K}^{27}$, Buckley $\mathrm{M}^{6}$, Murray $\mathrm{J}^{8}$, Lidral $\mathrm{A}^{28}$

${ }^{1}$ Division of Craniofacial Medicine, Department of Pediatrics, University of Washington, Seattle, USA

${ }^{2}$ Center for Developmental Biology \& Regenerative Medicine, Seattle Children's Research Institute, Seattle, USA

${ }^{3}$ Division of Basic Sciences, Fred Hutchinson Cancer Research Center, Seattle, USA ${ }^{4}$ Department of Anatomy \& Developmental Biology, Monash University, Melbourne, Australia

${ }^{5}$ Department of Orthodontics \& the Iowa Institute for Oral and Craniofacial Research, University of Iowa, Iowa City, USA

${ }^{6}$ New South Wales Health Pathology, Prince of Wales Hospital, Sydney, Australia

${ }^{7}$ Genetics of Learning Disability Service, Newcastle, Australia

${ }^{8}$ Department of Pediatrics, University of Iowa, Iowa City, USA

${ }^{9}$ Birth Defects Research Laboratory, University of Washington, Seattle, USA

${ }^{10}$ Division of Medical Genetics, Department of Medicine, University of Washington, Seattle, USA

${ }^{11}$ Division of Genetic Medicine, Department of Pediatrics, University of Washington, Seattle, USA

${ }_{12}$ Department of Medicine, Perelman School of Medicine, University of Pennsylvania, Philadelphia, USA

${ }^{13}$ Department of Genetics, Perelman School of Medicine, University of Pennsylvania, Philadelphia, USA

${ }^{14}$ Department of Biological Sciences, University of Delaware, Newark, USA

${ }^{15}$ Department of Genome Sciences, University of Washington, Seattle, USA

${ }^{16}$ Northwest Clinical Genomics Laboratory, Center for Precision Diagnostics,

University of Washington, Seattle, USA

${ }_{17}$ Department of Pathology, University of Washington, Seattle, USA

${ }^{18}$ Centre for Clinical Genetics, Sydney Children's Hospital, Randwick, Australia

${ }^{19}$ Seattle Craniofacial Center, Seattle Children's Hospital, Seattle, USA

${ }^{20}$ Centre for Molecular and Biomolecular Informatics, Radboud University Nijmegen

Medical Centre, Nijmegen, The Netherlands

${ }^{21}$ Fundación Clínica Noel, Medellin, 050021, Colombia

${ }^{22}$ South Australian Clinical Genetics Service, SA Pathology (at Women's and

Children's Hospital), North Adelaide, Australia

${ }_{23}$ School of Medicine, University of Adelaide, Adelaide, Australia

${ }^{24}$ Department of Human Genetics, Radboud University Nijmegen Medical Centre, Nijmegen, The Netherlands

${ }^{25}$ Department of Cognitive Neurosciences, Donders Institute for Brain, Cognition and Behaviour, Radboud University Medical Center, Nijmegen, The Netherlands

${ }^{26}$ Department of Molecular Developmental Biology, Radboud Institute for Molecular Life Sciences, Radboud University, Nijmegen, The Netherlands

${ }^{27}$ UVA Center for Advanced Medical Analytics, School of Medicine, University of Virginia, Charlottesville, USA

${ }^{28}$ Lidral Orthodontics, Rockford, USA

${ }^{29}$ Prince of Wales Clinical School, University of New South Wales, Randwick 2031,

Australia

${ }^{30}$ Neuroscience Research Australia, Randwick 2031, Australia

Purpose: Non-syndromic cleft lip/palate (NS-CL/P) is one of the most common human birth defects and is generally considered a complex trait. Despite numerous loci identified by GWAS, the effect sizes of common variants are relatively small with much of the genetic contribution remaining elusive and possible Mendelian causes not usually defined. Methods: We performed exome sequencing in 209 people from 72 multi-affected families using a Roche/Nimblegen SeqCap EZ v2.0 kit and samples were sequenced using the Illumina HiSeq 2500. Variants were annotated with the Ensembl Variant Effect Predictor and then filtered using GEMINI according to a standardized set of criteria to identify rare, pathogenic variants. Results: Pathogenic variants are described in four genes encoding components of the p120-catenin complex (CTNND1, PLEKHA7, PLEKHA5) and an epithelial splicing regulator (ESRP2), in addition to the known CL/P gene, CDH1. The findings were also validated in a second cohort of 497 people with NS-CL/P. Pathogenic variants in these genes were identified in the replication cohort in $14 \%$ of multi-affected and $2 \%$ of smaller families. Enriched expression of each gene/protein in human and mouse embryonic oro-palatal epithelia, demonstration of functional impact of CTNND1 and ESRP2 variants, and recapitulation of the CL/P 
spectrum in Ctnnd1 knockout mice support a causative role in CL/P pathogenesis. Conclusions: These data show that primary defects in regulators of epithelial cell adhesion are the most significant contributors to NS-CL/P identified to date and that inherited and de novo single gene variants explain a substantial proportion of NS-CL/P.

\section{Oral 3 \\ SIGNATURE OLIGOSACCHARIDES CHANGE THE WAY WE DIAGNOSE AND MONITOR THE MUCOPOLYSACCHARIDOSES}

Fletcher J', Bayly B', Saville J', Fuller $M^{\prime}$

${ }^{I}$ SA Pathology, North Adelaide, Australia

Introduction: The diagnosis of mucopolysaccharidoses traditionally relies on stepwise investigations, starting with the non-specific GAG assay, followed by electrophoresis, enzyme assay and mutation analysis. We have developed a new tandem mass spectrometric assay to detect signature oligosaccharides for each of the mucopolysaccharidoses. The assay is rapid, specific, sensitive and can be used for diagnosis and monitoring the effectiveness of therapies. Methods: The equivalent of $0.5 \mu$ mole urinary creatinine was evaporated to dryness and resuspended in derivatising reagent containing internal standard. Following incubation at $70 \mathrm{oC}$, and partial removal of the derivatising agent with chloroform, oligosaccharides were separated by liquid chromatography and analyzed on an AB SCIEX QTRAP 6500 triple quadrupole mass spectrometer, using multiple reaction monitoring for 10 targeted fragments plus the internal standard. Results: De-identified urines from 723 subjects, comprising 630 unaffected individuals and 93 MPS patients, were analyzed blinded to diagnosis. All 93 MPS samples were correctly identified by the presence of specific 'signature' oligosaccharides. Reference intervals were calculated from unaffected urines, with $99 \%$ confidence intervals, to yield an assay with $100 \%$ specificity and sensitivity. Conclusion: this test offers superior performance to 1- and 2-dimensional electrophoresis for diagnosis as well as monitoring the effectiveness of enzyme replacement, gene and stem cell therapies for the mucopolysaccharidoses. It has replaced standard testing in our, and two other Australian laboratories.

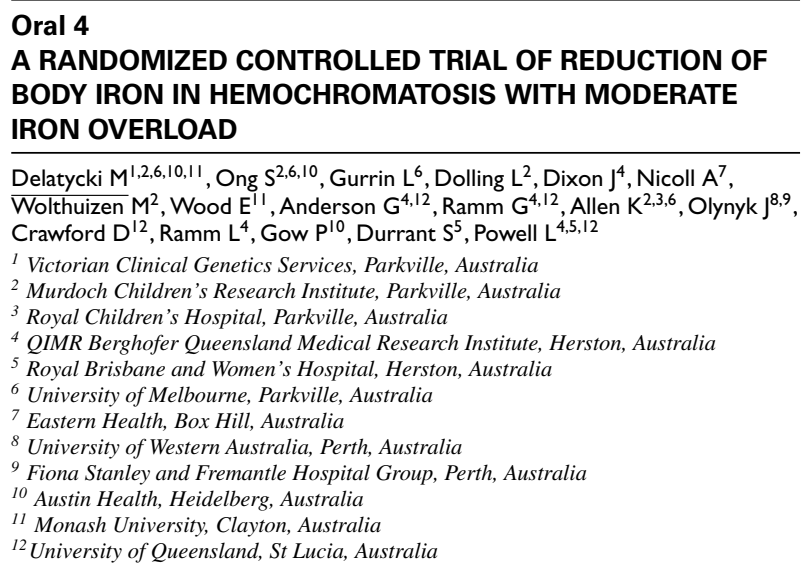

Background: Hereditary hemochromatosis ( $\mathrm{HH})$ is most commonly caused by HFE p.C282Y homozygosity. There is insufficient evidence to determine if individuals with $\mathrm{HH}$ and moderately elevated serum ferritin (SF), defined as SF $300-1000 \mu \mathrm{g} / \mathrm{L}$, should undergo iron reduction treatment. This study aimed to determine whether SF normalization improved symptoms and biochemical markers. Methods: HFE p.C282Y homozygotes, aged 18-70, with SF 300$1000 \mu \mathrm{g} / \mathrm{L}$ were randomized to either iron reduction by erythrocytapheresis (treatment group) or to sham treatment by plasmapheresis (control group). Patient-reported outcomes (Modified Fatigue Im- pact Scale (MFIS), Medical Outcomes Study Health Survey Version 2, Hospital Anxiety and Depression Scale and Arthritis Impact Measurement-2 Short-form Scale) and biochemical markers (Hepascore, Fibrometer, urine and plasma F2-isoprostanes and transient elastography) were measured at baseline and prior to unblinding. Findings: 104 participants were randomized to the treatment $(n=$ $54)$ and control $(n=50)$ groups, of whom 94 completed the study. Greater improvement in the primary outcome, MFIS score, occurred in the treatment group compared to the control group, (mean difference of $-6.25 S E 2.46,95 \%$ CI $[-11.1,-1.4] p=.01$ ) particularly in the cognitive subcomponent (mean difference of -3.60 SE 1.16, $95 \%$ CI $[-5.9,-1.3] p=.003)$. The hepatic fibrosis marker, Hepascore (mean difference of -0.05 SE $0.03,95 \%$ CI $[-0.1,0.0] p=.049$ ), and the oxidative stress marker, plasma F2-isoprostanes (mean difference -113.7 SE 53.9, 95\% CI [-220.9, -6.5] $p=.038$ ) showed greater reduction in the treatment than control group. Interpretation: This study suggests that SF normalization by iron depletion is indicated for all individuals with $\mathrm{HH}$ and elevated SF.

\section{Oral 5 \\ LONG-TERM ECONOMIC IMPACTS OF WHOLE EXOME SEQUENCING FOR SUSPECTED MONOGENIC DISORDERS \\ Schofield $D^{\prime}$, Rynehart L', Shresther $R^{\prime}$, White $S^{2,3}$, Stark $Z^{2,3}$ \\ ${ }^{I}$ GenIMPACT: Centre for Economic Impacts of Genomic Medicine, Department of Economics, Faculty of Business and Economics, Sydney, Australia \\ ${ }^{2}$ Victorian Clinical Genetics Services, Murdoch Children's Research Institute, Melbourne, Australia \\ ${ }^{3}$ Department of Paediatrics, University of Melbourne, Melbourne, Australia}

Background: Whole-exome and whole-genome sequencing (WES/WGS) has high diagnostic yield and clinical utility in rare genetic disease. However, the current health economic evidence base to support widespread adoption and reimbursement is very limited. Aim: To undertake the first cost-effectiveness analysis of WES for suspected monogenic disorders in comparison with usual diagnostic care, incorporating forecasting of both patient and family outcomes over a 20-year horizon. Methods: A cohort of 80 infants who underwent WES and usual diagnostic care in parallel were used to model incremental cost and health outcomes (using quality adjusted life years, QALYs) attributable to WES diagnosis. Three models were developed: (1) outcomes in patients only, (2) outcomes in patients and first-degree relatives as a result of cascade testing, (3) outcomes in patients and first-degree relatives including parental reproductive outcomes. Results: When the directly observed cost and health outcomes of the cohort participants were forecast over 20 years, the use of WES resulted in a total gain of 8.28 QALYs for the cohort and an incremental cost-effectiveness ratio (ICER) of $\$ 29,822.36$ per additional QALY gained. When cascade testing in first-degree relatives was added to the model, cost-effectiveness was substantially increased, generating a total gain of 20.33 QALYs and an ICER of $\$ 12,363.79$. When parental reproductive outcomes were added to the model, this produced the most cost-effective outcome, with a total QALY gain of 57.22 and an ICER of $\$ 8,679.62$. Discussion/Conclusions: WES in suspected monogenic disorders becomes more cost-effective as the benefits of cascade testing and reproductive outcomes are realized. 


\section{Oral 6}

\section{EXOMES OR GENOMES: DOES IT MATTER?}

Ewans $L^{1,2}$, Minoche $A^{2}$, Puttick $C^{2}$, Gayevskiy $V^{2}$, Drew $A^{2}$, Zhu $Y^{3,4}$, Walsh $C^{4}$, Ades $L^{5,6}$, Colley $A^{7}$, Ellaway $C^{6,8}$, Field $M^{3}$, Freckmann $M^{8,9}$, Goodwin $L^{10}$ Hackett $A^{3}$, Kamien $B^{11}$, Kirk $E^{4,8,12}$, Lipke $M^{8,13}$, Mowat $D^{8,12}$, Palmer $E^{3,12}$, Rajagopalan $S^{7}$, Ronan $A^{11}$, Sachdev $R^{8,12}$, Stevenson $W^{14}$, Turner $A^{8,12}$, Wilson $M^{5,6}$, Worgan $L^{7}$, Buckley $M^{4}$, Cowley $M^{1,2}$, Dinger $M^{1,2}$, Roscioli $T^{4,8,15^{\prime}}$

${ }^{1}$ St Vincent's Clinical School, University of New South Wales, Darlinghurst, Australia ${ }^{2}$ Kinghorn Centre for Clinical Genomics, Garvan Institute of Medical Research, Darlinghurst, Australia

${ }_{3}^{3}$ Genetics of Learning Disability Service, Waratah, Australia

${ }^{4}$ Randwick Genetics, NSW Health Pathology, Prince of Wales Hospital, Randwick, Australia

${ }^{5}$ Department of Clinical Genetics, Children's Hospital at Westmead, Westmead, Australia

${ }^{6}$ Disciplines of Child and Adolescent Health and Genetic Medicine, University of Sydney, Sydney, Australia

${ }^{7}$ Clinical Genetics Department, Liverpool Hospital, Liverpool, Australia

${ }^{8}$ Centre for Clinical Genetics, Sydney Children's Hospital, Randwick, Australia

${ }^{9}$ Clinical Genetics, Royal North Shore Hospital, St Leonards, Australia

${ }^{10}$ Genetics Services, Nepean Hospital, Nepean, Australia

${ }^{11}$ Hunter Genetics, Newcastle, Australia

12 School of Women's and Children's Health, University of New South Wales, Kensington, Australia

${ }^{13}$ Lady Cilento Children's Hospital, Brisbane, Australia

${ }^{14}$ Haematology Department, Royal North Shore Hospital, St Leonards, Australia

${ }^{15}$ Neuroscience Research Australia and Prince of Wales Clinical School, University of

New South Wales, Kensington, Australia

Purpose: Whole genome sequencing (WGS) can improve diagnostic rates over whole exome sequencing (WES) by accessing additional genomic variation, however the increased yield remains uncertain. We aimed to assess this diagnostic difference by undertaking WGS in families with Mendelian disorders that remained undiagnosed after WES. Methods: WGS was performed in a cohort of 100 individuals from 38 WES-negative families with various Mendelian disorders. In addition to small nuclear genomic variation, we sought structural and mitochondrial variation, complemented by homozygosity mapping to attain further diagnoses. Retrospective WES reassessment was performed to determine whether variants were diagnosable by contemporary WES or were unique to WGS analysis. Results: Overall our WGS-based pipeline identified diagnoses in over $30 \%$ of families in the cohort. Missed WES diagnoses were due to reduced coverage, older bioinformatics pipelines, inadequate literature evidence, or analyst interpretation. Preliminary retrospective investigation of WES data considering current bioinformatics pipelines and knowledge showed $20 \%$ of families could have attained a diagnosis. Thus, WGS resulted in additional diagnostic potential of $10 \%$, which may increase with the bulk of structural and mitochondrial analysis pending. We present clinically instructive cases of unexpected diagnoses highlighting the advantage of unbiased genomic testing approaches. Conclusion: WGS increased the diagnostic yield in WES negative families; however, a majority of diagnoses could have been identified using contemporary WES reanalysis. Genomic testing remains optimal for diagnosing Mendelian disorders, particularly when there is uncertainty in clinical diagnosis and will require ongoing laboratory-clinical collaboration and periodic data reanalysis to continue to improve the yield.

\section{Oral 7}

PRECISION CANCER GENOMICS TRIALS, FOR HIGH-RISK CHILDHOOD AND RARE ADULT CANCERS

Grady J', Wong $\mathrm{M}^{\prime}$, Hajdu $\mathrm{E}^{2}$, Mayoh $\mathrm{C}^{3}$, Mould $\mathrm{E}^{3}$, Barahona $\mathrm{P}^{3}$, Pinese $\mathrm{M}^{4}$, Gayevskiy $V^{1}$, Kumar $A^{5}$, Thavaneswaran $S^{4}$, Khuong-Quang $D^{6}, Q^{2} M^{7}$, Doig $K^{5}$, McEvoy $C^{5}$, Lukeis $R^{7}$, Prendergast $K^{3}, P a n G^{3}$, Collignon $E^{3}$, Sebastian $L^{8}$, Sjoquist $K^{8}$, Lee $C^{8}$, Fellowes $A^{5}$, Prawira $A^{4,9}$, Joshua $A^{4,9}$, Ballinger $M^{4}$, Lau $L^{3,10}$ Trahair $\mathrm{T}^{3,10}$, Fletcher $\mathrm{J}^{3}$, Arndt $\mathrm{G}^{3}$, Lock $\mathrm{R}^{3}$, Tyrrell $\mathrm{V}^{3}$, Dziadek $M^{\prime \prime}$, Fox $S^{5}$, Ziegler $D^{3,10}$, Dinger $M^{1,2,12}$, Mattick $J^{11,12}$, Ekert $P^{6}$, Marshall $G^{3,10}$, Haber $M^{3}$, Simes $]^{8}$, Thomas $D^{4}$, Cowley $M^{1,2,12}$

${ }^{1}$ Garvan Institute Of Medical Research, Darlinghurst, Australia

${ }^{2}$ Genome.One, Sydney, Australia

${ }^{3}$ Children's Cancer Institute, Sydney, Australia

${ }^{4}$ Cancer Division, Garvan Institute of Medical Research, Sydney, Australia

${ }_{5}^{5}$ Peter MacCallum Cancer Centre, Melbourne, Australia

${ }^{6}$ Murdoch Children's Research Institute, Melbourne, Australia

${ }^{7}$ SydPath St Vincent's Pathology, Sydney, Australia

${ }^{8}$ NHMRC Clinical Trials Centre, University of Sydney, Sydney, Australia

${ }^{9}$ The Kinghorn Cancer Centre, Sydney, Australia

${ }^{10}$ Kid's Cancer Centre, Sydney Children's Hospital, Sydney, Australia

${ }^{11}$ Garvan Institute of Medical Research, Sydney, Australia

${ }^{12}$ St Vincent's Clinical School, UNSW Sydney, Sydney, Australia

Background: Rare cancers, including pediatric cancers, account for $30 \%$ of cancer deaths. We hypothesized that genomic analysis of mutations underlying rare tumors would substantially improve patient outcomes, and generate disease insights. Aims: The main aims of this study were to determine the proportion of rare adult and childhood tumors with actionable mutations in their tumor and germline genomes and test the efficacy of consequent targeted therapies. Methods: Adults with rare cancers participated in the Molecular Screening and Therapeutics (MoST) program, where patients that have exhausted all conventional treatment options are recruited, and DNA + RNA from archival blocks are screened with the Illumina TST170 panel. Children with high-risk cancers participated in the Lions Kids Cancer Genome Project (LKCGP) / Zero Childhood Cancer (ZCC) program, which use WGS, RNA-Seq, targetedsequencing, in vitro and in vivo drug efficacy studies to comprehensively characterize each tumor. Results: Since August 2016, we recruited 571 patients into the MoST program, 55\% of whom had at least one actionable variant, and enrolled 105 onto targeted treatments, targeted+immunotherapy, or dual immunotherapy. We recruited 119 patients into the ZCC/LKCGP program: 53\% had relapsed disease, and over $40 \%$ had brain tumors. $43 \%$ of tumors had at least one actionable mutation, three with a change of diagnosis, and $6.5 \%$ with a reportable germline variant. Two tumors have near complete response following targeted therapy. Conclusion: Collectively the high rates of actionable variants identified in these rare cancers suggest substantial potential for improving outcomes using existing targeted therapies developed for other cancers.

\section{Oral 8}

\section{ASPREE: COHORT STRATIFICATION BY APOE GENOTYPE}

Riaz $M^{\prime}$, ASPREE Investigator Group, ASPREE Healthy Ageing Biobank, Ryan J', Huq $A^{1,2}$, Woods $R^{\prime}$, Storey $E^{\prime}$, Murray $A^{3}$, McNeil J', Lacaze $P^{\prime}$

${ }^{I}$ Department of Epidemiology and Preventive Medicine, School of Public Health and Preventive Medicine, Monash University, Melbourne, Australia

2 Department of Genetic Medicine, Royal Melbourne Hospital, Melbourne, Australia, ${ }^{3}$ Berman Center for Outcomes and Clinical Research, Minneapolis Medical Research Foundation, Minneapolis, USA

ASPirin in Reducing Events in the Elderly (ASPREE) is a longitudinal study of healthy ageing and Australia's largest clinical trial. ASPREE is comprised of 16,703 Australians aged over 70 years, and 2,411 Americans aged over 65 years, randomized to either daily low-dose aspirin or placebo to examine the preventative benefit of aspirin on a range of clinical outcomes. Approximately 15,000 ASPREE participants provided biospecimens, including consent for genetic studies, through the ASPREE Healthy Ageing Biobank. To date, the genomes of 11,541 APSREE participants have been sequenced using a targeted 'super panel' covering $>750$ genes used 
in clinical testing, including pan-cancer, cardiovascular, and neurological genes. Sequencing coverage is $\geq 97 \%$ for all bases, with an average read depth of $>200$. Here we present genotyping results for the Apolipoprotein E (APOE) gene, a major cholesterol carrier and strong genetic risk factor for Alzheimer's disease (AD) and cardiovascular disease (CVD). We stratify 11,541 ASPREE participants based on APOE genotype and identify $169(1.46 \%)$ APOE $\varepsilon 4 / \mathcal{E} 4$ homozygotes, average age 74 years. Despite significantly increased risk of $\mathrm{AD}$ and $\mathrm{CVD}$, these individuals had no prior history or clinical diagnosis of either at baseline, and passed a general cognition screening test within normal range ( $3 \mathrm{MS} \geq 78$ ). Absence of detectable disease symptoms, or delayed onset, despite carrying a significant genetic risk factor of high effect size, suggests the possibility of modifiers, or protective genetic factors in the genomes of these individuals, potentially contributing to protection against $\mathrm{AD}$ and/or CVD. Further studies are planned to elucidate such factors.

\section{Oral 9 \\ THE IMPACT OF GENETIC KNOWLEDGE ON INTENTIONS AND ATTITUDES OF THE COMMUNITY TOWARDS PRECONCEPTION CARRIER-SCREENING}

Ong $\mathrm{R}^{1,2}$, Howting $\mathrm{D}^{1,2}$, Rea $\mathrm{A}^{3}$, Christian $\mathrm{H}^{4}$, Charman $\mathrm{P}^{5}$, Molster $\mathrm{C}^{6}$, Ravenscroft $\mathrm{G}^{1,2}$, Laing $\mathrm{N}^{1,2}$

${ }^{1}$ Centre for Medical Research, University of WA, Australia

${ }^{2}$ Harry Perkins Institute of Medical Research, Australia

${ }^{3}$ Centre for Applied Statistics, University of WA, Australia

${ }_{4}$ School of Population and Global Health, and Telethon Kids Institute, Australia

${ }^{4}$ School of Population and Global Health, and Telethon Kids Institute, Australi
${ }_{5}^{5}$ Harry Perkins Institute of Medical Research, BioDiscovery Centre, Australia

${ }^{6}$ Department of Health, Australia

Background: Preconception carrier screening (PCS) provides the potential to empower couples to make reproductive choices before having an affected child. An important question is what factors influence the decision to utilize or not utilize preconception carrier screening. Methods: We analyzed the relationship between knowledge, attitudes and intentions to participate in PCS using logistic regression in 832 participants in Western Australia. Results: Twothirds of participants said they would take the test, with $92 \%$ of these supporting screening for diseases reducing the lifespan of children and infants. Those who had good genetic knowledge were seven times more likely to use PCS $(p \leq .001)$. While those with high genetic knowledge were four times more likely to use PCS ( $p$ $=.002$ ) and raised concerns such as insurance and confidentiality. Decreasing genetic knowledge correlated positively with religiosity and apprehension $(p \leq .001)$, which correlated negatively with intention to use PCS $(p \leq .001)$. Increasing genetic knowledge correlated positively with factors representing positive attitudes $p \leq$ .001 ), which correlated positively with intention to use PCS ( $p \leq$ .001). Many participants with good genetic knowledge nevertheless answered questions that tested understanding incorrectly. $80 \%$ of participants stated they would prefer to access the test through their general practitioners and $30 \%$ would pay up to AUD200. Conclusions: Knowledge is instrumental in influencing participation. Having good genetic knowledge may not be enough to understand core concepts of PCS and may impact informed decision-making. This study recommends that continuous education of health professionals and thus the community, in PCS is crucial to reduce misconceptions.

\section{Oral 10}

CELL-FREE FETAL DNA SCREENING IN SINGLETON IVF CONCEPTIONS

Lee $T^{1,3}$, da Silva Costa $F^{1,3}$, Rolnik $D^{3}$, McLennan $A^{4,5}$, Menezes $M^{1,2}$

${ }^{1}$ Monash Ultrasound For Women, Richmond, Australia

${ }^{2}$ Genetics Education and Health Research, Murdoch Childrens Research Institute, Parkville, Australia

${ }^{3}$ Department of Obstetrics and Gynaecology, Monash University, Clayton, Australia ${ }^{4}$ Sydney Ultrasound for Women, Sydney, Australia

${ }^{5}$ Discipline of Obstetrics, Gynaecology and Neonatology, University of Sydney, Sydney, Australia

Background: Cell-free DNA (cfDNA) screening has been shown to have high detection rates for trisomy 21 in the general obstetric population. Although uptake of cfDNA screening is high in women with IVF conceptions, focused and conclusive evidence regarding test performance/characteristics in the IVF population is lacking. Research Question: Are fetal fraction, test failure rate and positive predictive value (PPV) of cell-free fetal DNA (cffDNA) screening different in IVF conceptions compared to spontaneous conceptions? Methods: This was a retrospective cohort study including spontaneous and IVF-conceived singleton pregnancies that had cfDNA screening from April 2013 and November 2016. Multivariate regression analysis was used to determine significant predictors of logarithmically transformed fetal fraction and test failure. Comparison of test characteristics between study groups was performed adopting a significance level of 5\%. Results: 4633 spontaneously conceived and 992 IVF pregnancies were studied. Median fetal fraction was lower $(10.3 \%$ vs. $11.9 \% ; p=.005)$, test failure rate was higher $(5.2$ versus $2.2 \% ; p<.001)$ and PPV for trisomies 18,13 and SCA was poorer in IVF pregnancies compared to those spontaneously conceived. Multivariate linear regression analysis demonstrated that IVF conception, increased BMI, earlier gestational age and South and East Asian ethnicities were independent predictors of lower fetal fraction. Multiple logistic regression analysis found IVF conception and increased BMI to be independently associated with test failure. Discussion: Fetal fraction is significantly lower; test failure rate is higher and PPV is lower in IVF conceptions. These limitations should be taken into account when providing pre-test counseling for IVF conceptions.

\section{Oral 11}

PREVENTATIVE POPULATION GENOMIC SCREENING FOR ALL YOUNG ADULTS IN AUSTRALIA: A HEALTH-ECONOMIC ANALYSIS

Zhang L', Bao Y', Riaz $M^{\prime}$, Tiller J', McNeil J', Lacaze P'

${ }^{I}$ School of Public Health and Preventive Medicine, Monash University, Melbourne, Australia

Background: Preventative impact and cost-effectiveness of population genomic screening (PGS) for multiple conditions concurrently in early adulthood is unknown. Here we model impact of offering preconception carrier screening and cancer predisposition gene testing to all young adults in Australia in a single test, following standard of care and reimbursed health services, compared to targeted testing. Methods: In 2,688,192 individuals, all adults aged 1825 years, we model births affected by cystic fibrosis, spinal muscular atrophy and fragile $\mathrm{X}$ syndrome using carrier frequencies, and incidence of breast, ovarian, colorectal and endometrial cancer attributable to BRCA1/BRCA2/MLH1/MSH2 mutations. We then consider population screening at $70 \%$ uptake, with impact measured by affected births prevented and reduced cancer (incidence/mortality). We calculate incremental cost-effectiveness ratio (ICER, cost/disability-adjusted life year (DALY) gained), at per-sample screen costs of AUD $\$ 200 / \$ 400 / \$ 800 / \$ 1200$ versus targeted testing. Results: Assuming conservative intervention uptake, PGS would significantly reduce incidence and mortality beyond 
current targeted testing. PGS would be cost-effective, below the willingness-to-pay threshold AUD\$50,000/DALY, up to a test cost of AUD $\$ 1200$ in this population. Assuming AUD\$400 per-test, total cost of PGS, including subsequent confirmatory testing, counseling and ongoing clinical surveillance for mutation carriers, would be AUD $\$ 600 \mathrm{M}$ above current testing. But PGS would avert $\geq 70,000$ DALYs and save $\geq$ AUD $\$ 300 \mathrm{M}$ in treatment costs, being highly cost-effective versus targeted testing (ICER $\leq$ AUD \$5,000/DALY). If AUD\$200 per test were achieved, investment would reduce to $\$ 387 \mathrm{M}$, making screening cost-saving to the health system (ICER $\leq$ AUD\$0/DALY). Conclusion: PGS for multiple conditions concurrently in early adulthood would be highly cost-effective, potentially cost-saving, for the Australian system.
Concurrent Session 1 - Australasian Society of Diagnostic Genomics ASDG Oral 1 CLINICAL UTILITY OF NON-INVASIVE PRENATAL TESTING (NIPT) FOR THE DETECTION OF SEGMENTAL ANEUPLOIDY

Flowers $N^{\prime}$, Giouzeppos $O^{\prime}$, Shi G', Love $C^{\prime}$, Tsegay $A^{\prime}$, Harrington $T^{\prime}$, Manser $\mathrm{R}^{\prime}$, Burns $I^{\prime}$, Baeffel $S^{\prime}$, Archibald $A^{\prime, 2}$, Scarff $K^{\prime}$, Hunt $C^{\prime}$, Pertile $M^{1,2}$

${ }^{I}$ Victorian Clinical Genetics Services, Murdoch Children's Research Institute, Parkville, Australia, ${ }^{2}$ Department of Pediatrics, University of Melbourne, Parkville, Australia

Background: The scope of NIPT has expanded beyond screening for common autosomal trisomies and sex chromosome aneuploidies. Whole genome sequencing data from NIPT can be interrogated for segmental aneuploidies. Aim: We assessed the clinical utility and possible clinical performance of reporting segmental aneuploidies as additional findings, when identified during whole chromosome screening for rare autosomal trisomies. Methods: We retrospectively analyzed 15,600 cfDNA results for possible segmental aneuploidies and gathered available cytogenetic outcome data, where a cytogenetic abnormality had been confirmed following pre- or postnatal investigations. Whole chromosome cfDNA analysis was performed using WISECONDOR v2.0.0. SNP microarray and FISH were the primary tools for cytogenetic investigations. Results: $18 / 15600(0.1 \%)$ were screen positive for a segmental aneuploidy, 11/18 (61\%) were true positives. The true positives observed included deletions associated with Cri du Chat syndrome and Wolf Hirschhorn syndrome, and a Miller-Dieker Lissencephaly deletion, associated with an unbalanced translocation. The 7 false positives included maternal findings as well as confined placental mosaicism for the segmental aneuploidy. Within the cohort 3 false negative results were identified. Two of these cases were complicated by placental mosaicism for a normal cell line and the other case by low fetal fraction. Discussion/Conclusion: Screening pregnancies for segmental aneuploidies at NIPT enables the detection of clinically significant genomic imbalances that would otherwise go unrecognized. Detecting these segmental aneuploidies at the time of NIPT allows for greater pregnancy management options for patients and clinicians. The false negative cases are instructive of the limitations of screening cfDNA for segmental aneuploidies in the fetus.

\section{ASDG Oral 2 \\ CLINICAL UTILITY OF WHOLE GENOME SEQUENCING COPY NUMBER VARIANT DETECTION WITH NO LOWER SIZE LIMITS}

Lundie $B^{1,2,3}$, Minoche $A^{\prime}$, Gayevskiy $V^{1}$, Statham $A^{2}$, Hobbs $M^{2}$, Ewans $L^{1,2,3}$, Hollway $G^{1,2,3}$, Ohnesorg $T^{2}$, Sherstyuk $A^{2}$, Dinger $M^{1,2,3}$, Burnett $L^{2,3,4}$, Cowley $M^{1,3}$

${ }^{1}$ Garvan Institute of Medical Research, Kinghorn Centre for Clinical Genomics, Darlinghurst, Australia

${ }^{2}$ Genome. One, Darlinghurst, Australia

${ }^{3}$ St Vincent's Clinical School, UNSW, Darlinghurst, Australia

${ }^{4}$ Sydney Medical School, University of Sydney, St Leonards, Australia

Genome.One has recently completed clinical validation of ClinSV, an analysis pipeline that identifies regions of copy number variation $(\mathrm{CNV})$ from whole genome sequencing (WGS) data, utilising evidence from split-reads, discordant pairs and depth-of-coverage to obtain a comprehensive, high confidence CNV call-set. Clinical validation was performed against microarray, MLPA and NA12878 gold standard. No lower limit for size was applied, allowing unprecedented resolution of CNV. Analytical sensitivity, assessed against NA12878, was $98.0 \%$ for deletions $>500$ bp and $84.1 \%$ for deletions $<500 \mathrm{bp}$. Results were $100 \%$ concordant with reportable variants previously detected by microarray. To identify variants undetectable by other methods, and to determine the clinical utility of high-resolution genome-wide $\mathrm{CNV}$ analysis, we have begun a retrospective review of previously reported, uninformative WGS cases 
$(n=161)$ using ClinSV. In addition, CNV analysis is now a standard part of Genome.One's clinical WGS service. Analysis of the first 20 cases of the retrospective study has already yielded diagnostic CNVs, including a 1kb homozygous deletion of SPG7 exon 6 in a patient with spastic paraplegia, and an exon 1 DST deletion combined with a stop-gain (nonsense) variant in two deceased neonates with unknown neuromuscular disorder. We will present our validation experiments and results from retrospective reviews to illustrate the incremental utility of CNV analysis. We demonstrate that WGSbased genome-wide $\mathrm{CNV}$ analysis has wider breadth and higher resolution than any other test available and increases WGS diagnostic yield.

\section{ASDG Oral 3 \\ UNIPARENTAL DISOMY IN PREGNANCIES WITH RARE AUTOSOMAL TRISOMIES IDENTIFIED USING WHOLE-GENOME cfDNA SCREENING}

Pertile $M^{\prime, 2}$, Flowers $N^{\prime}$, Giouzeppos $O^{\prime}$, Shi $G^{\prime}$, Tsegay $S^{\prime}$, Harrington $T^{\prime}$, Burns $I^{\prime}$, Manser $R^{\prime}$, Baeffel $S^{\prime}$, Archibald $A^{\prime, 2}$, Scarff $K^{\prime}$, Hunt $C^{\prime}$, Oertel $R^{\prime}$, Norris $F^{\prime}$

${ }^{I}$ Victorian Clinical Genetics Services, Murdoch Children's Research Institute, Parkville, Australia

${ }^{2}$ University of Melbourne, Department of Paediatrics, Parkville, Australia

Background: We report our experience of using genome-wide cfDNA screening to identify rare autosomal trisomies with evidence of uniparental disomy (UPD) in the fetus. Methods: Rare autosomal trisomies (those other than 13, 18 and 21) were identified in pregnancies screened by cfDNA analysis between April 2015 and September 2017 at the VCGS, Melbourne. The ratio of the trisomic fraction of cfDNA relative to fetal fraction (TF/FF) was calculated for each rare trisomy identified. Amniocentesis was offered in viable on-going pregnancies. Prenatal diagnosis was usually by single nucleotide polymorphism (SNP) microarray and included UPD analysis when the rare trisomy involved an imprinted chromosome. Results: Rare trisomies were detected in 114 of 33,764 cfDNA samples $(0.34 \%)$. In 105 pregnancies with outcome data, 45 were associated with early pregnancy loss. In the remaining 60 pregnancies, PND was performed or the pregnancy continued without cytogenetic investigations. SNP microarray and UPD studies identified 13 pregnancies with putative or confirmed UPD involving chromosomes 2 ( 2 cases $), 4$ ( 4 cases), 8 ( 1 case), 12 ( 1 case), 15 ( 1 case), 16 ( 3 cases) and 22 ( 1 case). Four UPD cases were also complicated by TFM, indicating partial trisomy rescue. Another case of trisomy 2 was identified with mosaic uniparental isodisomy confined to the placenta in a severely growth restricted fetus. Conclusion: Rare autosomal trisomies identified using cfDNA screening are frequently associated with UPD arising from complete or partial trisomy rescue. High $\mathrm{TF} / \mathrm{FF}$ ratio can be used to identify pregnancies at increased risk for UPD and residual trisomy mosaicism.

\section{ASDG Oral 4 \\ A GENOMIC AUTOPSY OF PERINATAL DEATH: BEYOND THE HUMAN GENOME}

Byrne $A^{1,2,7}$, Arts $P^{1,7}$, Walker $M^{2}$, Weyrich $L^{3}$, Feng $J^{1,7}$, Soubrier $J^{4}$, Schreiber $A^{1,3,7}$, Babic $M^{1}$, Khong $Y^{5}$, Moore $L^{3,5}$, MacArthur $D^{2}$, Hahn $C^{1,3,7}$, Kassahn $K^{3,4}$, King-Smith $\mathrm{S}^{1,6}$, Barnett $\mathrm{C}^{3,5}$, Scott $\mathrm{H}^{1,3,4,6,7}$

${ }^{1}$ Centre for Cancer Biology, Adelaide, Australia

${ }^{2}$ Broad Institute of MIT and Harvard, Boston, USA

${ }^{3}$ University of Adelaide, Adelaide, Australia

${ }^{4}$ SA Pathology, Adelaide, Australia

${ }^{5}$ Women's and Children's Hospital, Adelaide, Australia

${ }^{6}$ Australian Genomics Health Alliance, Melbourne, Australia

${ }^{7}$ University of South Australia, Adelaide, Australia

Pregnancy loss or newborn death are devastating events, and precise identification of the cause is essential for accurate counseling. Congenital abnormalities are the leading cause of death $(33 \%)$, followed by infection (15\%); however, the etiology of malformations is not always apparent and a causative agent cannot always be determined in cases with infectious pathology. Here, we examine the utility of a 'genomic autopsy' in identifying microbial species causative of fetal death. Whole genome sequencing is being performed on DNA extracted from fetal lung, with different protocols trialled to optimize dual extraction of human and microbial DNA. Sequence reads are mapped to a set of microbial reference genomes and a metagenomics approach employed to determine microbial load and identify causative organisms. A proof-of-principal study has been performed on 20 cases, 10 due to infection (positive) and 10 due to congenital abnormality without a molecular cause (negative). Causative microbes were correctly identified in 4/7 known positive cases and plausible microbes identified for $2 / 3$ unsolved positive cases. Methods are now being optimized to improve diagnostic yield. As expected, no significant microbial load was identified in 9/10 negative cases, however, a known pathogen was identified in 1 case. Analysis of human-mapping reads also revealed a genetic variant in 1 positive case. Upon review, the patient's phenotype shared similarities to that expected for the gene. Together, the dual analysis of human and microbial DNA that a genomic autopsy allows may facilitate an accurate cause of death to be established in up to $50 \%$ of cases.

\section{ASDG Oral 5}

\section{DETECTION OF EMBRYO MOSAICISM WITH NGS}

Farrell $M^{\prime}$, Grkovic $S^{\prime}$, Stockton J', McArthur $S^{\prime}$

${ }^{l}$ Genea, Sydney, Australia

The field of Pre-implantation Genetic Screening has embraced Next Generation Sequencing (NGS) due to improved accuracy, increased sample throughput and reduced costs compared to microarrays. However, NGS has brought new challenges due to the detection of whole and segmental chromosome mosaic aneuploidy in 15-20\% of embryos. The aim was to internally validate reproducibility and technical limits of NGS for mosaicism detection and investigate the prevalence of mosaicism in the remainder of the embryo. 56 mosaic samples were resequenced to investigate possible artifact introduction during NGS library preparation. To determine detection limits, samples with known aneuploidies were titrated against normal samples. Rebiopsies of the same embryo were conducted to investigate prevalence of mosaicism in the rest of the embryo. Sample resequencing showed $100 \%$ concordance, confirming the reproducibility of NGS. Limits of mosaicism detection for gains or losses in whole chromosomes were found to be $10 \%$. For segmentals, detection limits ranged from $20 \%$ for large ( $>25 \mathrm{MB}$ ) to $50 \%$ for small ( $<10 \mathrm{MB}$ ) fragments. Rebiopsy confirmed mosaicism in 4/5 embryos for whole chromosomes, with reciprocal changes observed in 3 of these. Only $3 / 8$ segmentals were confirmed, with wide variability in the observed abnormalities suggesting an alternative mechanism of origin. This study provided assurance of NGS technical detection limits, which enables confident classification of samples as mosaic. The results confirmed that the distribution, type and frequency of mosaicism is unpredictable throughout an embryo. The frequent detection of mosaic whole chromosome reciprocal changes provides support for suggestions that monosomic and trisomic mosaics should be ranked equally.

\section{ASDG Oral 6 \\ PRENATAL MICROARRAY ANALYSIS: ARE ALL THE CHALLENGES RELATED TO VARIANTS OF UNCERTAIN SIGNIFICANCE?}

Woodward K', Stampalia J', Taylor $F^{\prime}$, Sivamoorthy $S^{\prime}$, Potts $K^{\prime}$, Chionh $A^{\prime}$, Grumball $\mathrm{T}^{\prime}$, Beilby J', Azmanov D'

${ }^{I}$ Pathwest Laboratory Medicine WA, Perth, Australia

Prenatal chromosome microarray analysis (CMA) is still developing as a routine diagnostic test and utilization is now extending 
beyond pregnancies with ultrasound abnormalities. However, there are different approaches to service integration, data analysis and reporting and not all are simply related to the various technical platforms used. We describe our experiences with prenatal microarray over the last 3 years and discuss the associated challenges. Using detailed analysis of copy number variants (CNV) in regions associated with serious morbidity and mortality and lower backbone resolution $(3 \mathrm{Mb})$, the level of detected abnormalities was consistent with the literature (10\% pathogenic $\mathrm{CNV}$ and $5 \%$ variants of uncertain significance (VOUS)). However, this approach is likely to miss clinically relevant variants as $19 \%$ of our reported variants were identified through an additional visual inspection of the data (mostly mosaic CNV). The level of reported VOUS was significantly reduced by the targeted analysis/reporting approach; however, as susceptibility loci were also not reported, withheld information may have had postnatal implications. While the sensitivity of microarray has been superior to conventional karyotype analysis, parallel culture set-up has been helpful in resolving complexities in $23 \%$ of abnormal cases. Simultaneous maternal microarray analysis has helped in confirmation of prenatal sample ID; however, this posed challenges for reporting maternally inherited CNV. The robustness of microarray compared with conventional cytogenetics has launched the technology to the prenatal diagnostic forefront. Yet, other contemporary technologies such as non-invasive prenatal screening and diagnostic massively parallel sequencing will continue to challenge the utility of microarray.

Concurrent Session 2 - Australasian Society of Inborn
Errors of Metabolism
ASIEM Oral 1
PERSONALIZED GENOMIC MEDICINE - THE DIETETIC
ROLE

Rani H. Singh

Department of Human Genetics, Emory University, Decatur, Georgia, USA

New discoveries in the field of genetics and recent developments in newborn screening has increased exponentially and created both opportunities and challenges for clinicians. To a large extent, this has been due to new 'omic' technologies like tandem mass spectrometry (MS/MS) that have facilitated large-scale analysis of the metabolic profile at a public health level as Newborn Screening (NBS) program. This has resulted in several-fold increase in the number of tests and represents the largest volume of genetic testing. In addition, cost-effective sequencing has led to an explosion of discoveries of novel genetic mutations that not only reveal heterogeneity by assessing relationships between gene defects and disease severity but also the potential to understand genotype-phenotype relationships. By understanding the integrated 'omics' on identified patients through NBS, we now have the opportunity to intervene presymptomatically and in a targeted manner with nutritional and therapeutic interventions. Number of-related metabolite-gene-diet interactions in the area of inherited metabolic disorders of clinical importance are now evident, supporting the emerging field of nutrigenetics for meaningful conclusions that may eventually lead targeted dietary and other treatment recommendations. While placing increasing demands on clinicians to plan appropriate nutritional interventions, such discoveries should ultimately revolutionize clinical care as we move into the predictive, preventive, and personalized era of genomic medicine. Newborn screening and nutrition intervention offer a great example of nutrigenetics with a far-reaching potential in the prevention of some poor outcomes of many genetic disorders and influence on health promotion and disease prevention on a global scale. The future of Genetic metabolic nutrition is unquestionably linked to newborn screening.

\section{ASIEM Oral 2}

\section{A TASTE OF YOUR OWN MEDICINE}

Elliott $\mathrm{A}^{\mathrm{I}}$

${ }^{1}$ Lady Cilento Children's Hospital, Brisbane, Australia

In 2018 the Metabolic Dietary Disorders Association (MDDA) launched 'The great protein challenge'. A challenge to reduce protein intake to $10 \mathrm{~g}$ for 24 hours in support of phenylketonuria (PKU). Queensland Metabolic Lifespan Service was keen to demonstrate support and better understand what patients experience daily. Method: Four dietitians and two nurses participated in a two-day challenge. Day 1: 4 staff consumed $10 \mathrm{~g}$ protein plus manufactured low protein (MLP) foods, 1 had no MLP foods, Day 2: 5 staff consumed $10 \mathrm{~g}$ protein, MLP foods plus $60 \mathrm{~g}$ protein from PKU Formula, 1 had no MLP foods. The hospital provided a $3 \mathrm{~g}$ protein lunch on both days. Participants were surveyed 1 day post challenge. Results: 3 out of 5 were not satisfied on the LP diet only. $100 \%$ were satisfied on the LP diet with PKU Formula. Several formula were consumed including low volume and GMP. Most found the formula difficult to complete with a pleasant initial flavour but unpleasant, repeating aftertaste. Popular LP foods included LP pasta, cookies, bread mixes, meatballs and cheese. Unpopular were packaged bread and milk substitute. Positive experiences included a good range of LP foods and not being hungry when also on formula. Negative experiences included time spend worrying about food, reading labels, missing out at social functions and nausea with formula. Conclusion: Participants found it a valuable experience that will support practice. Despite the issues with formula, $100 \%$ would complete the challenge again. We raised $\$ 1,446$ for MDDA which we hope to improve upon next year.

\section{ASIEM Oral 3 \\ ADDRESSING PRACTICALITIES OF METABOLIC RESTRICTED DIETS THROUGH COOKING DEMONSTRATIONS TO CHILDREN AND FAMILIES}

Slack $S^{1}$, Mitchell $A^{\prime}$, Bhattacharya $K^{2,3}$, Thompson $S^{2}$

${ }^{I}$ Department of Nutrition \& Dietetics, the Children's Hospital at Westmead, Sydney, Australia

${ }^{2}$ Genetic Metabolic Disorders Service, The Children's Hospital at Westmead, Sydney, Australia

${ }^{3}$ Disciplines of Child and Adolescent Health and Genetic Medicine, University of Sydney, Sydney, Australia

Background: The restrictive nature of metabolic diets can provide a number of challenges for pediatric patients and their families; including learning how to use specialized products, cooking with limited ingredients, dietary variety, social implications and their relationship with food. Aim: Provide an overview of cooking demonstrations offered to patients/families at The Children's Hospital at Westmead. Present feedback and anecdotal experiences of patients involved in the sessions. Method: Review of numbers and types of cooking demonstrations conducted since September 2015. Retrospective audit of diet notes for feedback or reported changes, from cooking demonstrations conducted in 2018. Results: 55 cooking demonstrations have been conducted since Sept 2015; 6 groups of children and 49 individual ( 20 to children, 29 to parents), with 30 sessions to patients with PKU and 25 to patients with a range of other metabolic diagnoses. Feedback from all recent cooking sessions ( $n$ $=7$ ) was positive with reported benefits including; improved dietary intake or variety, repeating recipes at home $(5 / 7)$ or 'more open to trying new foods'. The demonstrations focusing on using specialized low protein products helped parents feel more confident in using these products and relieved some anxiety; for example, low protein baking is 'less daunting having seen it being made'. Discussion: Cooking demonstrations provide practical support for patients and their families/carers to assist with adherence to therapeutic diets. Many recipients have found them helpful in providing support to 
challenges faced with their restrictive diets. Formal evaluation and needs assessment is required to investigate the benefits and determine areas for improvement.

\section{ASIEM Oral 4 \\ ESSENTIAL FATTY ACID DEFICIENCIES IN ADULT PKU PATIENTS ON LOW PROTEIN DIETS}

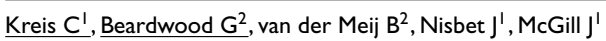 \\ ${ }^{I}$ Queensland Lifespan Metabolic Service-Mater Group, South Brisbane, Australia \\ ${ }^{2}$ Facility of Health Sciences \& Medicine, Bond University, Robina, Australia}

People with PKU are at risk of essential fatty acid deficiency (EFAD) due to dietary restriction of high EFA sources in the treatment diet and inadequate supplementation in some prescribed PKU formulas. Barriers such as poor formula compliance, and periods of rapid growth can heighten the EFAD risk. This investigation aims to identify the proportion of adults with PKU who have EFAD and to detect variables that are associated with EFAD. Retrospective pathology data was collected from 2011-2017 for adults with PKU. Results retrieved included EFA profiles, with mean blood phenylalanine from 6- and 12- months prior to EFA collection to assess compliance to the PKU treatment diet and formula. Data on age, gender, pregnancy status, anthropometry, formula type and dose were also obtained. Data were collated and analyzed using Chi-square tests to identify if differences in EFAD exist across variables. Full data sets were collected for 124 EFA samples from 51 adults with PKU. Of these EFA samples, $27 \%(n=33)$ showed one or more EFAD. Further data analysis revealed that $36.4 \%(n=12)$ of the identified EFAD were from pregnant women. Of these pregnancies $76 \%(n=$ 9) were compliant with prescribed dose of PKU formula and protein restricted diet. Despite pregnant women with PKU maintaining high compliance with low protein diet and formula, EFAD do readily exist during this rapid growth phase. This investigation stimulates identification and improved dietary management of EFAD in adults with PKU and encourages further research in this area.

\section{ASIEM Oral 5 \\ CHALLENGES IN ACHIEVING GOOD METABOLIC CONTROL IN A TEENAGER WITH GYRATE ATROPHY NEW TO AUSTRALIA}

van der Haak $N^{\prime}$, Sweeney $A^{\prime}$, Ketteridge $D^{\prime}$, Naveen $D^{\prime}$, Bratkovic $D^{\prime}$

${ }^{I}$ Women's And Children's Hospital, Adelaide, Australia

Background: A female teenager with gyrate atrophy as a result of ornithine aminotransferase deficiency migrated to Australia in November 2017. Plasma ornithine in her home country was reportedly stable between $400-500 \mu \mathrm{mol} / \mathrm{L}$. Since moving to Australia, she reported deterioration in vision. She disliked low protein foods available in Australia, had an irregular eating pattern and plasma ornithine fluctuated between $691-857 \mu \mathrm{mol} / \mathrm{L}$. Treatment approaches in Australia including different combinations of reducing natural protein from $0.4 \mathrm{~g} / \mathrm{kg} / \mathrm{day}$ to $0.15 \mathrm{~g} / \mathrm{kg} /$ day, increasing Essential Amino Acid supplement (EAA) from $0.2 \mathrm{~g} / \mathrm{kg} / \mathrm{day}$ to $0.4 \mathrm{~g} / \mathrm{kg} /$ day and encouraging regular meals failed to reduce plasma ornithine levels. Aims: To reduce plasma ornithine levels in a female teenager with gyrate atrophy new to Australia. Methods: A 5-day admission was arranged. The treatment plan included $10 \mathrm{~g}$ of natural protein $(0.15 \mathrm{~g} / \mathrm{kg} /$ day $), 6 \times 5 \mathrm{~g}$ EAA $(0.4 \mathrm{~g} / \mathrm{kg} /$ day $)$ and low protein foods available in Australia spread across the day to provide a total protein intake of $0.55 \mathrm{~g} / \mathrm{kg} /$ day. Plasma ornithine levels were checked twice daily. Results: Fasting ornithine reduced from $636 \mu \mathrm{mol} / \mathrm{L}$ on admission to $569 \mu \mathrm{mol} / \mathrm{L}$. Ornithine 2 hours after supper reduced from $588 \mu \mathrm{mol} / \mathrm{L}$ to $455 \mu \mathrm{mol} / \mathrm{L}$. The diet plan was strictly followed with $10 \mathrm{~g}$ of natural protein, $6 \times 5 \mathrm{~g}$ EAA and an average of 1,984 calories consumed each day. Conclusion: A very low natural protein diet with EAA, low protein foods and adequate calorie intake reduced plasma ornithine levels. This case study highlights the importance of working closely with patients new to Australia to achieve a treatment plan that meets meal preferences while also achieving good metabolic control.

Concurrent Session 3 - Australasian Association of Clinical Geneticists

AACG Oral 1

NAD DEFICIENCY, CONGENITAL MALFORMATIONS AND NIACIN SUPPLEMENTATION

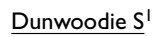

${ }^{I}$ Victor Chang Cardiac Research Institute, Darlinghurst, Australia

Congenital malformations can manifest as combinations of phenotypes that co-occur more often than expected by chance. In many such cases it has proved difficult to identify a genetic cause. We sought the genetic cause of cardiac, vertebral and renal defects, among others, in 13 unrelated patients. Variants were identified in 3-hydroxyanthranilic acid 3,4-dioxygenase (HAAO) and kynureninase (KYNU) genes encoding kynurenine pathway enzymes. Three patients carried homozygous variants predicting loss-of-function changes in the HAAO or KYNY proteins (HAAO p.D162*, HAAO p.W186*, or KYNU p.V57Efs*21). Another patient carried heterozygous KYNU variants (p.Y156* and p.F349Kfs*4). Recurrent miscarriage also occurred in some of these families. The mutant enzymes had greatly reduced activity in vitro. Nicotinamide adenine dinucleotide (NAD) is synthesized de novo from tryptophan via the kynurenine pathway. The patients had reduced circulating NAD levels. Haao or Kynu null mouse embryos developed similar defects to the patients due to NAD deficiency. In null mice, averting NAD deficiency during niacin supplementation during gestation prevented the malformations and miscarriage.

\section{AACG Oral 2 \\ WHOLE-GENOME SEQUENCING FOR PATIENTS WITH UNCLASSIFIED LEUKODYSTROPHIES.}

Stutterd $C^{1,2,3,4}$, Delatycki $M^{1,3,4}$, Lockhart $P^{1,4}$, Taft $R^{5,8}$, Vanderver $A^{6,7}$, Simons $\mathrm{C}^{8,9}$, Leventer $\mathrm{R}^{2,4,10}$

${ }^{I}$ Bruce Lefroy Centre for Genetic Health Research, Murdoch Children's Research Institute, Parkville, Australia

${ }^{2}$ Department of Neurology, Royal Children's Hospital, Parkville, Australia

${ }^{3}$ Victorian Clinical Genetics Service, Parkville, Australia

${ }^{4}$ Department of Paediatrics, University of Melbourne, Parkville, Australia

${ }^{5}$ Illumina Inc, San Diego, USA

${ }^{6}$ Division of Neurology, Children's Hospital of Philadelphia, Philadelphia, USA

${ }^{7}$ Perlman School of Medicine, University of Pennsylvania, Philadelphia, USA

${ }^{8}$ Institute for Molecular Bioscience, University of Queensland, St. Lucia, Australia

${ }^{9}$ Translational Bioinformatics Research group, Murdoch Children's Research Institute, Parkville, Australia

${ }^{10}$ Neuroscience Research Group, Murdoch Children's Research Institute, Parkville, Australia

Background: Leukodystrophies are a genetically diverse group of disorders that have in common the selective involvement of the central nervous system (CNS) white matter. They most commonly present in childhood and follow a progressive course, with high morbidity and mortality and limited life span. A genetic diagnosis is key to providing accurate prognostic information and reproductive counseling to families and appropriate clinical management of the patient. Magnetic resonance imaging (MRI) pattern recognition and exome sequencing currently achieve a diagnosis for $70 \%$ of patients. Aim: To identify the genetic causes underlying a cohort of patients with unclassified leukodystrophy on MRI or negative whole exome sequencing (WES). Methods: Trio/Quad whole-genome sequencing (WGS) was performed on 28 patients from 23 families with leukodystrophy and a non-diagnostic MRI pattern or non-diagnostic WES. Results: A genetic diagnosis was achieved in 11/23 families $(47 \%)$. Implicated genes included six typically associated with leukodystrophy: DARS2, NDUFV1, BOLA3, COL4A1, TUBB4A, 
SLC17A5 and five genes not previously-associated with leukodystrophy: HMBS, FIG4, STAG2, SCN2A, SCN8A. The latter group includes genes associated with porphyria, epileptic encephalopathy, peripheral neuropathy and congenital malformation. Trio WGS was effective in identifying de novo variants as well as variants that had been missed by exome sequencing due to poor coverage. Conclusions: Results from this cohort advocate the use of trio WGS for the diagnosis of CNS white matter disease as the genetic etiologies are diverse and may include de novo dominant developmental genes as well as recessive housekeeping, metabolic and mitochondrial genes.

\section{AACG Oral 3 \\ IDENTIFYING NOVEL DISEASE GENES IN GENETICALLY UNDIAGNOSED PATIENTS WITH RETT SYNDROME}

Kaur $S^{1,2}$, Van Bergen $N^{1,2}$, Gold $W^{3,4}$, Howden $S^{5}$, Dottori $M^{6}$, Kupier $M^{7}$, Miller $D^{8}$, Roscioli $T^{8}$, Eggers $S^{9,10}$, Lunke $S^{9,10}$, Stark $Z^{2,10}$, Ellaway $C^{4,11}$, Friez $M^{12}$, Ben Zeev $B^{13}$, Torella $A^{14}$, Nigro $V^{14}$, Pinner $J^{15}$, Sachdev $R^{15}$, Milnes $D^{16}$, Schonewolf-Greulich B $B^{17}$, Tümer $Z^{17}$, Christodoulou J,2,3,4

${ }^{I}$ Neurodevelopmental Genomics Research Group, Murdoch Children's Research Institute, Parkville, Australia

${ }^{2}$ Department of Paediatrics, University of Melbourne, Parkville, Australia

${ }^{3}$ Metabolic Research, Kids Research Institute, The Children's Hospital at Westmead, Westmead, Australia

${ }^{4}$ Discipline of Genetic Medicine and Child and Adolescent Health, Sydney Medical

School, University of Sydney, Camperdown, Australia

${ }^{5}$ Kidney Development, Disease and Regeneration, Murdoch Children's Research

Institute, Parkville, Australia

${ }^{6}$ Illawarra Health and Medical Research Institute, University of Wollongong,

Wollongong, Australia

${ }^{7}$ Data61, CSIRO, Parkville, Australia

${ }^{8}$ Garvan Institute, Darlinghurst, Australia

${ }^{9}$ Translational Genomics Unit, Murdoch Children's Research Institute, Parkville, Australia

${ }^{10}$ Victorian Clinical Genetics Services, Murdoch Children's Research Institute,

Parkville, Australia

${ }^{\prime \prime}$ Western Sydney Genetics Program, The Children's Hospital at Westmead, Westmead, Australia

${ }^{12}$ Greenwood Genetic Center, Greenwood, USA

13 Sheba Medical Center, Tel HaShomer, Israel

${ }^{14}$ Department of Precision Medicine, University of Campania 'Luigi Vanvitelli', Caserta, Italy

${ }_{15}$ Centre for Clinical Genetics, Sydney Children's Hospital, Randwick, Australia

${ }^{16}$ Genetic Health Queensland, Royal Brisbane \& Women's Hospital, Herston, Australia

${ }^{17}$ Kennedy Center, Department of Clinical Genetics, Copenhagen University Hospital, Rigshospitalet, Denmark

Rett Syndrome (RTT) is a severe disorder of neuronal plasticity, mainly caused by mutations in the X-linked methyl-CpG-binding (MECP2) gene. About $95 \%$ of patients with classical RTT yet only $60 \%$ atypical RTT patients have mutations in MECP2, so a significant proportion of patients remain genetically undiagnosed. Our focus is to identify novel candidate genes using next generation sequencing techniques in genetically undiagnosed RTT patients. We used whole genome, whole exome sequencing or a custom targeted sequencing panel to screen a cohort of 39 genetically undiagnosed RTT/ RTT-like patients. In one patient we identified a novel de novo missense variant in KIF1A, encoding a neuronal specific kinesin molecular motor transporter essential for axonal trafficking of synaptic cargos. Subsequently, various missense variants were identified in eight more patients. In silico analysis predicted all of these variants in the motor domain of protein to be pathogenic. Another patient is heterozygous for a variant in KIF23 which regulates dendritic microtubule trafficking. A homozygous missense variant in KCTD16, exclusively expressed in brain and coding an auxiliary protein for GABA receptor assembly was also found. A homozygous variant in a well-conserved site of the 3' untranslated region of MECP2 predicted to affect microRNA binding site was also uncovered. A range of functional assays are being designed to confirm the pathogenicity of the variants using both immortalized neuronal cell lines and patient-specific neuronal cells differentiated from induced pluripotent cells. This work has potentially identified new genes associated with RTT and provided unique insights into underlying biological process.

\section{AACG Oral 4 \\ MODELLING PRECONCEPTION SCREENING USING WHOLE GENOME SEQUENCING DATA FROM 1,144 WELL ELDERLY AUSTRALIANS}

Kirk $E^{1,2,3}$, Challis $B^{4}$, Pinese $M^{5}$, Rath $E^{5}$, Roscioli $T^{1,2,6}$, Thomas $D^{5}$

${ }^{1}$ Sydney Children's Hospital, Randwick, Australia

${ }^{2}$ New South Wales Health Pathology, Randwick, Australia

${ }^{3}$ School of Women's and Children's Health, UNSW, Randwick, Australia

${ }^{4}$ University of Newcastle, Newcastle, Australia

${ }^{5}$ Garvan Institute, Darlinghurst, Australia

${ }^{6}$ Neuroscience Research Australia, Randwick, Australia

Background: Massively parallel sequencing makes preconception screening for almost all known autosomal recessive (AR) and Xlinked (XL) conditions possible. The Medical Genome Reference Bank (MGRB) consists of whole genome sequencing (WGS) data from $\sim 4,000$ healthy older Australians. Aim: We sought to identify reportable variants in genes associated with $\mathrm{AR}$ and XL disorders in the MGRB, to determine aggregate carrier frequencies and the probability that a couple from this population would be at high risk of having a child affected by a severe condition. Methods: 2,819 genes associated with AR or XL phenotypes were identified using annotations in OMIM, and WGS data from 1,144 individuals were filtered against the following criteria: (1) Allele frequency $<0.02$ in population databases, (2) Loss of function OR having at least one ClinVar annotation of Likely Pathogenic or Pathogenic. Results: 1,751 unique variants remained after filtering. These were hand-curated using relevant elements of the ACMG pathogenicity criteria. On average, 1.69 variants assessed as at least likely pathogenic were identified per person. Couples drawn from this population would have a $1.83 \%$ chance of being at risk of AR conditions and $0.58 \%$ for $\mathrm{XL}$ conditions. However, some of the conditions are relatively mild and would be unlikely to be included in a preconception screening program. Conclusion: We found an overall carrier burden per person of 1.69 , and estimate that $2 \%$ of couples would be at risk of having a child affected by a severe AR or XL condition. 


\section{AACG Oral 5 \\ DE NOVO VARIANTS DISRUPTING THE HX-REPEAT MOTIF OF ATN1 CAUSE A RECOGNIZABLE CONGENITAL NEUROCOGNITIVE DISORDER}

Palmer $\mathrm{E}^{1,2,3,4}$, Hong $\mathrm{S}^{5}$, AlZahrani $\mathrm{F}^{6}, \mathrm{O}$ Hashem $\mathrm{M}^{6}$, Aleisa $\mathrm{F}^{5}$, Jalal Ahmed $\mathrm{H}^{5}$ Kandula $T^{2,3}$, Macintosh $R^{2}$, Minoche $A^{4}$, Puttick $C^{4}$, Gayevskiy $V^{4}$, Drew $A^{4}$, Cowley $M^{4,7}$, Dinger $M^{4,7}$, Rosenfeld $]^{8}$, Cho $M^{9}$, Henderson $L^{9}$, Guillen Sacoto $M^{9}$, Begtrup $A^{9}$, Hamad $M^{10}$, Shinawi $M^{11}$, Andrews $M^{11}$, Jones $M^{12}$, Lindstrom $\mathrm{K}^{13}$, Kayani $\mathrm{S}^{14}$, Snyder $M^{15}$, Villanueva $M^{16}$, Schteinschnaider $A^{16}$, Roscioli $T^{2,18,19}$, Kirk $E^{2,3,18}$, Bye $A^{2,3}$, Merzaban $J^{5}$, Jaremko $L^{5}$, Jaremko $M^{5}$, Sachdev $R^{2,3}$, Alkuraya $\mathrm{F}^{6,17,20}$, Arold $\mathrm{S}^{5}$

${ }^{1}$ Genetics of Learning Disability Service, Waratah, Australia

${ }^{2}$ Sydney Children's Hospital, Randwick, Australia

${ }^{3}$ School of Women's and Children's Health, University of New South Wales, Randwick, Australia

${ }^{4}$ Garvan Institute, Darlinghurst, Australia

${ }^{5}$ King Abdullah University of Science and Technology (KAUST), Thuwal, Saudi Arabia

${ }^{6}$ King Faisal Specialist Hospital and Research Center, Riyadh, Saudi Arabia

${ }^{7}$ St Vincent's Clinical School, UNSW, Darlinghurst, Australia

${ }^{8}$ Baylor College of Medicine, Houston, USA

${ }^{9}$ GeneDx, Gaithersburg, USA

${ }^{10}$ King Khalid University Hospital, Saudi Arabia

${ }^{11}$ Washington University School of Medicine, St. Louis, USA

${ }^{12}$ San Diego and Rady Children's Hospital, San Diego, USA

${ }^{13}$ Phoenix Children's Hospital, Phoenix, USA

${ }^{14}$ University of Texas Southwestern Medical Center, Dallas, USA

${ }^{15}$ Children's Health, Dallas, USA

${ }^{16}$ Fundación para la Lucha contra las Enfermedades Neurológicas de la Infancia,

Montañeses, Buenos Aires, Argentina

${ }_{17}^{17}$ Alfaisal University, Riyadh, Saudi Arabia

${ }^{18}$ Randwick Genetics, NSW Health Pathology, Randwick, Australia

${ }^{19}$ Neuroscience Research Australia and Prince of Wales Clinical School, Randwick, Australia

${ }^{20}$ King Abdulaziz City for Science and Technology, Riyadh, Saudi Arabia

Polyglutamine expansions in ATN1, encoding a transcriptional corepressor, have been linked to the neurodegenerative condition dentatorubral-pallidoluysian atrophy (DRPLA) via a proposed toxic gain of function. We present detailed phenotypic information on seven unrelated individuals with de novo missense and insertion variants within an evolutionarily conserved 16 amino acid 'poly HX repeat' motif of ATN1. Each of the subjects has severe cognitive impairment and hypotonia, recognisable facial gestalt and variable congenital anomalies. However, they lack of progressive symptoms typical of DRPLA neurodegeneration. To distinguish this subset of patients from the DRPLA diagnosis, we suggest using the term congenital ATN1-related disorder (CARD) to classify the condition. We show that a recurrent CARD-related variant is sufficient to perturb the structural features of ATN1's HX repeat. This alters ligand binding, including the binding to several proteins which are important in ribosomal function and regulation of gene expression. In addition, it affects the nuclear localization of the HX repeat motif. These data suggest that the variant affects the transcriptional repression activity of ATN1. Our study provides valuable insights into the function of the HX repeat motifs and ATN1's primary roles regulating neuronal and other organ system development. This is another example of phenotypically distinct allelic disorders in humans, similar to our recent findings for ZSWIM6-related disorders (MIM 617865) and reveals the power of unbiased genomic technologies and international collaborations in providing diagnoses for individuals with complex congenital disorders.

\section{AACG Oral 6 \\ STRATEGIES FOR DISEASE GENE DISCOVERY IN FAMILIAL AND SPORADIC AMYOTROPHIC LATERAL SCLEROSIS AND FRONTOTEMPORAL DEMENTIA}

Fifita $J^{\prime}$, McCann $E^{\prime}$, Chan Moi Fat $S^{\prime}$, Williams $K^{\prime}$, Twine $N^{\prime, 2}$, Bauer $D^{2}$, Kowk $\vec{J}^{3}$, Halliday $\mathrm{G}^{3}$, Kiernan $\mathrm{M}^{3}$, Rowe $\mathrm{D}^{1}$, Nicholson $\mathrm{G}^{1,4,5}$, Blair $\mathrm{I}^{1}$

${ }^{1}$ Centre For MND Research, Macquarie University, Australia

${ }^{2}$ Commonwealth Scientific and Industrial Research Organization, Health \&

Biosecurity Flagship, North Ryde, Australia

${ }^{3}$ Brain and Mind Centre, University of Sydney, Sydney, Australia

${ }^{4}$ Northcott Neuroscience Laboratory, ANZAC Research Institute, Concord, Australia

${ }^{5}$ Sydney Medical School, University of Sydney, Sydney, Australia

Amyotrophic lateral sclerosis (ALS) is a fatal neurodegenerative disease caused by progressive degeneration of the upper and lower motor neurons. Approximately $10 \%$ of ALS cases are hereditary (familial), with the remaining $90 \%$ being sporadic. Around $20 \%$ of ALS patients exhibit co-morbid frontotemporal dementia. Gene mutations remain the only established cause of ALS. However, mutations account for only two thirds of familial and $10 \%$ of sporadic ALS cases. We aim to identify novel causative or disease modifying variants in ALS families and sporadic ALS/FTD patients. A novel genetic, in silico and in vitro analysis pipeline was developed for gene discovery in familial ALS. Using this pipeline, custom analysis of whole-exome sequencing data from three members of an Australian ALS family negative for known ALS mutations identified five candidate gene variants. Subsequently, only one of these variants showed strong pathogenic evidence after in silico and in vitro analyses. Whole-genome sequencing has been completed on an Australian cohort of 635 sporadic ALS, and 108 FTD cases. Custom bioinformatics scripts were developed to rapidly analyze known ALS/FTD genes in this dataset. Initial analysis identified four novel variants in two known ALS genes, and a burden of nonsynonymous variants in one gene in sporadic ALS cases compared to controls. This project aims to identify novel ALS/FTD mutations, reveal the prevalence of known mutations in patients, and identify genetic variation that may modify disease phenotype. By elucidating genetic contributors to ALS/FTD we hope to enhance our understanding of disease and to aid studies for therapeutic development.

\section{Concurrent Session 4 - Australasian Society of Genetic Counsellors \\ ASGC Oral 1 \\ HELPING YOUNG CHILDREN UNDERSTAND AN INHERITED CANCER PREDISPOSITION SYNDROME USING BIBLIOTHERAPY}

Schlub G', Crook $A^{2}$, Flemming J', O'Shea R', Barlow-Stewart K', Tucker $K^{3}$, Greening $S^{4}$

${ }^{I}$ Genetic Medicine, Sydney Medical School - Northern, University of Sydney, Royal North Shore Hospital, St Leonards, Australia

${ }^{2}$ Department of Clinical Genetics, Royal North Shore Hospital, St Leonards, Australia ${ }^{3}$ Hereditary Cancer Clinic, Prince of Wales Hospital, Randwick, Australia

${ }^{4}$ Hereditary Cancer Clinic, Illawara Cancer Care Centre, Wollongong, Australia

Background: Communication with children about hereditary conditions is a process for which parents often feel ill equipped. Yet, good communication strategies are leading determinants of adaptation and resilience. In the case of hereditary phaeochromocytoma and paraganglioma syndrome (HPPS), which results in rare neuroendocrine tumors, malignancy and an early age of onset ( $>5$ years), genetic testing and subsequent surveillance in at-risk children is the optimal intervention. Given the young age at which this commences, great effort is needed to provide these children and their parents with appropriate genetic counseling and communication strategies. Aim: By adopting a bibliotherapeutic framework - where stories are delivered prescriptively (i.e., 'bibliotherapy'), develop and evaluate the first psycho-educational resource in Australia for children at risk of HPPS. Methods: An illustrated 
storybook was created based on models of developmental comprehension and given to parents of children aged 5-10 years old at risk of HPPS recruited through genetics services. Semi-structured qualitative interviews were conducted and analyzed thematically using a general inductive approach to ascertain parents' experience reading this storybook with their children, including recommendations for improvement. Results and Significance: Five parents have been interviewed, and recruitment is continuing. Unanimously, parents reported this resource would have been useful had it been available when their children were first tested and/or diagnosed. We anticipate that by incorporating parental views, this cost effective and easily deployable resource, will have psycho-educational benefit that may improve the process of communication between parents and children about HPPS with possible further cross-application to other inherited pediatric cancer syndromes.

\section{ASGC Oral 2 \\ EVALUATING A TELEPHONE GENETIC COUNSELING SERVICE FACILITATING BRCA1/2 TESTING FOR WOMEN WITH OVARIAN CANCER}

\author{
Forrest $L^{1,2}$, Tutty $E^{1}$, McKinley $J^{1}$, Forbes Shepherd $R^{1,2}$, James $P^{1,2}$, Meiser $B^{3}$, \\ Young $M^{4}$ \\ ${ }^{I}$ Peter MacCallum Cancer Centre, Melbourne, Australia \\ ${ }^{2}$ The University of Melbourne, Melbourne, Australia \\ ${ }^{3}$ The University of New South Wales, Sydney, Australia \\ ${ }^{4}$ Genome One, Darlinghurst, Australia
}

PARP inhibitors offer a survival advantage to women with highgrade serous ovarian cancer who have a germline BRCA1/2 mutation. However, uptake of BRCA1/2 testing in this population is low. In 2016, a centralized, national telephone genetic counseling (TGC) service was established to address barriers to testing. This study aimed to explore the experience, acceptability, and feasibility of the TGC service. Women who received TGC from January 2016 to May 2017 were invited to complete a survey. Survey domains, based on validated measures, included: previous access to genetic services, satisfaction with TGC, and decision-making about BRCA1/2 testing. 107 women completed the survey (response rate 48\%); 60\% resided in Queensland, eight had a BRCA1/2 mutation. Oncologists were the preferred provider of BRCA1/2 testing. Prior access to genetic services was limited, and participants used TGC to overcome geographical barriers. Despite $46 \%$ of the women making an informed choice to have testing, $72 \%$ had no regret. The impact of receiving genetic results, while low overall, was greater for BRCA1/2 carriers than non-carriers $(z=2.64, p=.008)$. All respondents demonstrated high levels of satisfaction with the TGC they received. Women's preferred provider of BRCA1/2 testing was their oncologist; supporting mainstreaming from a consumer perspective. This preference did not appear to impact women's satisfaction with TGC, and none regretted their decision to have BRCA1/2 testing. Disclosure of genetic test results had a low emotional impact, particularly on BRCA1/2 non-carriers. TGC appears to be an acceptable way to reduce barriers to BRCA1/2 testing in Australia.

\author{
ASGC Oral 3 \\ GENETICS AND INSURANCE IN AUSTRALIA \\ Tiller J', Lacaze $P^{1}$, Delatycki $M^{2,3,4}$, Otlowski $M^{5}$, Keogh $L^{6}$, Morris $S^{7}$, Rice $T^{7}$, \\ Barter $\mathrm{K}^{8}$ \\ ${ }^{1}$ Public Health Genomics, Monash University, Melbourne, Australia \\ ${ }^{2}$ Victorian Clinical Genetics Services, Parkville, Australia \\ ${ }^{3}$ Bruce Lefroy Centre for Genetic Health Research, Parkville, Australia \\ ${ }^{4}$ Murdoch Children's Research Institute, Parkville, Australia \\ ${ }^{5}$ Faculty of Law, University of Tasmania, Hobart, Australia \\ ${ }^{6}$ Centre for Health Equity, Melbourne School of Population and Global Health, The \\ University of Melbourne, Carlton, Australia \\ ${ }^{7}$ Lynch Syndrome Australia, The Summit, Australia \\ ${ }^{8}$ Pink Hope Australia, Brookvale, Australia
}

The use of genetic test results by insurance companies in underwriting has increasingly been the subject of Australian regulatory scrutiny over the past 12 months. The 2017 parliamentary inquiry into the life insurance industry, which released its official report and recommendations in March 2018, highlighted challenges with industry self-regulation and the emerging prevalence of genetic discrimination by insurance companies. The report made recommendations for a moratorium on the use of genetic test results in life insurance in Australia, but as at April 2018 this has yet to be implemented. Although anecdotal evidence of genetic discrimination at the hands of life insurers in Australia does exist, systematic or quantitative documentation of individuals' experiences of discrimination or denial of insurance has been lacking. In this study, we collected survey data from over 200 individuals with cancer-predisposing mutations for either hereditary breast and ovarian cancer (provided by Pink Hope) or Lynch syndrome (provided by Lynch Syndrome Australia) to gauge the scale of the problem. Results demonstrate dozens of documented cases of individuals reporting difficulties accessing life insurance products as a result of positive test results for cancerpredisposing mutations. In some cases, individuals have been denied life insurance or offered significantly increased premiums despite undertaking significant risk-reducing surgery or participating in intensive screening programs. This presentation will present unpublished data, provide updates regarding the outcomes of the Parliamentary Inquiry, and consider political and regulatory steps now required for the implementation of the recommended moratorium in Australia.

\section{ASGC Oral 4 \\ RARE AUTOSOMAL TRISOMIES DETECTED BY NON-INVASIVE PRENATAL TESTING; IMPORTANT AND NOT SO RARE \\ Scott $\mathrm{F}^{\mathrm{l}, 2}$, Bonifacio $\mathrm{M}^{3}$, Sandow $\mathrm{R}^{\mathrm{I}}$, Ellis $\mathrm{K}^{3}$, Smet $\mathrm{E}^{\mathrm{l}}$, McLennan $\mathrm{A}^{\mathrm{I}, 4}$ \\ ${ }_{2}^{1}$ Sydney Ultrasound for Women, Sydney, Australia \\ ${ }^{2}$ University of New South Wales, Kensington, Australia \\ ${ }^{3}$ Genea Pathology, Sydney, Australia \\ ${ }^{4}$ University of Sydney, Camperdown, Australia}

Non-invasive prenatal testing (NIPT) can assess chromosomes other than 13, 18, 21, X and Y. These rare autosomal trisomies (RATs) can adversely affect pregnancy outcome. A prospective study of NIPT using the Illumina sequencing platform assessing all chromosomes was conducted. There were 28 RATs identified in 23,388 samples ( 1 in 835$)$, the most common being trisomy $7(n=6)$, followed by trisomy $16(n=4)$ and trisomy $22(n=3)$. Abnormal outcomes occurred in 16 cases; miscarriage $(n=6)$, true fetal mosaicism $(n=5)$ and fetal structural anomaly on ultrasound $(n=$ $5)$. Growth restriction was seen in 8 cases and correlated with very low pregnancy-associated plasma protein-A (PAPP-A) levels. Two of the 17 live-born babies had a structural anomaly, and one had a phenotype similar to mosaic trisomy 16 despite a normal microarray result. RATs are not rare and are often associated with poor obstetric outcomes. They should be discussed with the clinician to guide management. Pregnancy outcomes varied by chromosome, being 
generally favourable for some (e.g., trisomy 7) and poor for others (eg. trisomy 22). In the presence of a RAT, PAPP-A is predictive of placental dysfunction and fetal growth restriction.

\section{ASGC Oral 5 \\ CHANGING INDICATIONS FOR PRENATAL GENETICS SERVICES: IMPLICATIONS FOR GENETIC COUNSELING PRACTICE IN A TERTIARY CENTRE}

\author{
Graetz $M^{1,2}$, Kelley J ${ }^{1,2}$, Hui L', Dao $C^{1,2}$ \\ ${ }^{I}$ Mercy Perinatal, Mercy Hospital for Women, Heidelberg, Australia \\ ${ }^{2}$ GeNE at the Mercy Hospital, Mercy Hospital for Women, Heidelberg, Australia
}

Aim: Prenatal genetic testing has been transformed by technological advances, including noninvasive prenatal testing (NIPT), chromosomal microarrays and specialized fetal imaging. Our aim was to analyze the indications for referral to genetic counseling in our tertiary maternity hospital over a 10 year period, and reflect on implications for genetic counselors. Methods: Numbers and indications for genetic counseling referrals from 2006 to 2016 were retrieved from hospital databases, medical records, genetic files and procedural logbooks. Referrals were categorized into a single major indication for referral, including fetal ultrasound abnormality, increased risk screening test result, advanced maternal age, family history and prior history of pregnancy with a genetic condition. Statistical significance was analyzed using the chi-squared test for proportions or trend as appropriate. Results: Referrals for fetal structural abnormalities increased significantly from $17 \%$ (83/486) in 2006, to $20 \%(118 / 580)$ in 2011 and 34\% (174/452) in $2016(p<.0001)$. In contrast, referrals for an increased risk screening result decreased from $27 \%$ (181/486) in 2006 to $32 \%$ (187/580) in 2011 and to $23 \%$ $(104 / 452)$ in $2016(p=.001)$. Abnormal results from prenatal diagnosis increased steadily from $4 \%(13 / 294)$ in 2006 , to $10 \%(28 / 286)$ in 2011 and $25 \%(47 / 187)$ in $2016(p<.0001)$. Conclusion: The patient population referred to our genetic counseling service has increased in acuity and complexity over the past decade. The increased number of women receiving a diagnosis of a fetal/chromosome abnormality has important implications for service provision. Supervision and self-care is essential for genetic counselors to manage the increasingly demanding nature of the role.

\section{ASGC Oral 6 PREIMPLANTATION GENETIC DIAGNOSIS IN INHERITED HEART DISEASES: A QUALITATIVE STUDY}

Yeates $L^{1,3}$, McDonald $K^{1}$, Burns $C^{1,2,3}$, Semsarian $C^{1,2,3}$, Ingles $J^{1,2,3}$

${ }^{I}$ Agnes Ginges Centre for Molecular Cardiology, Centenary Institute, Sydney, Australia ${ }^{2}$ Sydney Medical School, University of Sydney, Sydney, Australia

${ }^{3}$ Department of Cardiology, Royal Prince Alfred Hospital, Sydney, Australia

Introduction: Preimplantation genetic diagnosis (PGD) is a reproductive technique that ensures a pathogenic variant is not passed to the next generation. PGD is known to cause significant emotional burden. Inherited heart diseases show variable penetrance and clinical heterogeneity, ranging from asymptomatic individuals to heart failure and sudden cardiac death. Here we explore the experiences of PGD in the setting of inherited heart diseases. Method: Participants were recruited from a specialized multidisciplinary cardiac genetic clinic. Purposive sampling was used. Patients and partners who had previously considered and/or undertaken PGD were invited to participate. A semi-structured interview schedule was developed to explore overall experiences and reasons for PGD uptake. Broad topics included experience of disease, reproductive history, psychosocial and financial considerations. Interviews were recorded, transcribed verbatim and thematic analysis performed. Results: 11 participants were recruited (10 with an inherited cardiomyopathy and 1 partner). Two broad themes emerged: (1) Past experience influencing now: encompassing patients experience of disease, reproductive history and personal beliefs; and (2) Deliberating the decision: including uncertainty for self and future generations, judgment from others, isolation and financial considerations. Among those who chose to undergo PGD (7/11), past experience of a significant cardiac event, such as family history of sudden cardiac death, was an important factor in the decision process. Conclusion: The decision to undergo PGD in inherited heart disease is complex and influenced by individual experience of disease. We highlight key areas where genetic counseling intervention may assist in PGD decision processes.

\section{Concurrent Session 5 - Australasian Society of Diagnostic Genomics \\ ASDG Oral 7 \\ A FUNCTIONAL GENOMICS RESEARCH PROGRAM SUPPORTING CLINICAL MOLECULAR DIAGNOSIS: SPLICE SITE VARIANT ANALYSIS}

Riley $L^{1,2}$, Bournazos $A^{3}$, Sandaradura $S^{2,3}$, Ghaoui $R^{2,3,4}, M a A^{5,6}$, Cooper $S^{3,7}$

${ }^{I}$ Rare Diseases Functional Genomics, Kids Research, The Children's Hospital at Westmead and The Children's Medical Research Institute, Sydney, Australia

${ }^{2}$ Discipline of Child and Adolescent Health, Sydney Medical School, University of Sydney, Sydney, Australia

${ }_{3}^{3}$ Kids Neuroscience Centre, The Children's Hospital at Westmead, Sydney, Australia

${ }^{4}$ Royal Adelaide Hospital, Department of Neurology, Adelaide, Australia

${ }^{5}$ Department of Clinical Genetics, Children's Hospital Westmead and Nepean Hospital, Sydney, Australia

${ }_{6}^{6}$ Discipline of Genetic Medicine, Children's Hospital Westmead Clinical School, Sydney Medical School, University of Sydney, Sydney, Australia

${ }^{7}$ Functional Neuromics Group, The Children's Medical Research Institute, Sydney, Australia

Background: We have established a functional genomics research program to support clinical diagnosis of rare diseases. Functional analysis of variants of uncertain significance can provide experimental evidence enabling reclassification as a likely pathogenic variant following ACMG guidelines. Here we present three cases where mRNA studies have established likely pathogenicity of noncoding, putative splice variants, leading to a definitive molecular diagnosis. Method: cDNA studies were performed on a homozygous ANO10 c.1163-9A > G variant found in an individual with cerebellar ataxia; compound heterozygous ABCB4 (c.344+2_3insTT and c. $2064+3 \mathrm{~A}>\mathrm{T}$ ) variants in siblings with intrahepatic cholestasis; and a heterozygous DES c.1289-741G $>$ A variant in trans with a c.569T >C p.Leu190Pro variant in siblings with muscular dystrophy and cardiomyopathy. RNA extracted from blood or muscle was analyzed by RT-PCR and agarose gel electrophoresis, then Sanger sequenced. Results: The ANO10 c.1163-9A > G variant ablated normal splicing, inducing exon 7 skipping or use of a cryptic splice acceptor, with both events resulting in a frameshift. The ABCB4 c.344+2_3insTT variant promoted skipping of exon 5 (out of frame) and the c.2064+3A $>\mathrm{T}$ variant caused exon 16 skipping (in frame). The DES c.1289-741G $>$ A variant created a cryptic splice acceptor leading to inclusion of a 118 nucleotide pseudo-exon, effecting a frameshift. Conclusion: Functional mRNA analyses of four splice site variants provided experimental evidence showing splicing abnormalities with clear damaging effects for the gene. These data enabled classification as likely pathogenic according to ACMG criteria, providing a definitive molecular diagnosis for three families with rare diseases. Our study highlights the value of research support for clinical diagnostics. 


\section{ASDG Oral 8 \\ A SCREENING APPROACH TO IDENTIFY CLINICALLY ACTIONABLE VARIANTS CAUSING CONGENITAL HEART DISEASE IN EXOME DATA}

Szot $J^{1,2}$, Cuny $\mathrm{H}^{1,3}$, Blue $\mathrm{G}^{4,5}$, Humphreys $\mathrm{D}^{1,3}$, Ip $E^{1,3}$, Harrison $\mathrm{K}^{6}$, Sholler $\overline{G^{4,5,7}}$, Giannoulatou $E^{1,3}$, Leo $P^{8}$, Duncan $E^{8,9,10}$, Sparrow $D^{1,3}$, Ho $J^{1,3}$, Graham $\mathrm{R}^{1,3}$, Pachter $\mathrm{N}^{6,11}$, Chapman $\mathrm{G}^{1,3}$, Winlaw $\mathrm{D}^{4,5}$, Dunwoodie $\mathrm{S}^{1,2,3}$

${ }^{1}$ Victor Chang Cardiac Research Institute, Sydney, Australia

${ }^{2}$ UNSW, Faculty of Science, Sydney, Australia

3 UNSW, Faculty of Medicine, Sydney, Australia

${ }^{4}$ The Children's Hospital at Westmead, Heart Centre for Children, Sydney, Australia

5 Sydney Medical School, University of Sydney, Sydney, Australia

${ }^{6}$ Genetic Services of Western Australia, Perth, Australia

${ }^{7}$ Sydney Children's Hospitals Network, Sydney, Australia

${ }^{8}$ Institute of Health and Biomedical Innovation, Faculty of Health, QUT, Brisbane, Australia

${ }^{9}$ Department of Endocrinology and Diabetes, Royal Brisbane and Women's Hospital, Brisbane, Australia

${ }^{10}$ Faculty of Medicine, University of Queensland, Brisbane, Australia

${ }^{11}$ School of Paediatrics and Child Health, UWA, Perth, Australia

Background: Congenital heart disease (CHD), structural abnormalities of the heart that arise during embryonic development, is the most common inborn malformation, affecting $\leq 1 \%$ of the population. However, currently, only a minority of cases can be explained by genetic abnormalities. The goal of this study was to identify disease-causal genetic variants from whole-exome data in 30 families affected by CHD. Methods: We utilized a 2-tiered whole-exome variant screening and interpretation procedure. First, we manually curated a high-confidence list of 90 genes known to cause CHD in humans, identified predicted-damaging variants in genes on this list, and rated their pathogenicity using American College of Medical Genetics and Genomics-Association for Molecular Pathology guidelines. Results: In 3 families (10\%), we found pathogenic variants in known CHD genes TBX5, TFAP2B, and PTPN11, explaining the cardiac lesions. Second, exomes were comprehensively analyzed to identify additional predicted damaging variants that segregate with disease in CHD candidate genes. In 10 additional families (33\%), likely disease-causal variants were uncovered in PBX1, CNOT1, ZFP36L2, TEK, USP34, UPF2, KDM5A, KMT2C, TIE1, TEAD2, and FLT4. Conclusions: The pathogenesis of CHD could be explained using our high-confidence CHD gene list for variant filtering in a subset of cases. Furthermore, our unbiased screening procedure of family exomes implicates additional genes and variants in the pathogenesis of CHD, which suggest themselves for functional validation. This 2-tiered approach provides a means of (1) identifying clinically actionable variants and (2) identifying additional disease-causal genes, both of which are essential for improving the molecular diagnosis of CHD.

\section{ASDG Oral 9 \\ A NOVEL METHOD TO IDENTIFY PATHOGENIC REPEAT EXPANSIONS IN EXOME OR GENOME SEQUENCE DATA}

Bahlo $M^{1,2}$, Tankard $R^{1,2}$, Bennett $M^{1,2}$, Degorski $P^{1,2}$, Delatycki $M^{3,4}$, Lockhart $\mathrm{P}^{3,5}$

${ }^{1}$ Walter and Eliza Hall Institute of Medical Research, Parkville, Australia

${ }^{2}$ Department of Medical Biology, University of Melbourne, Parkville, Australia

${ }^{3}$ Victorian Clinical Genetics Services, Parkville, Australia

${ }^{4}$ Bruce Lefroy Centre for Genetic Health Research, Murdoch Children's Research Institute, Parkville, Australia

${ }^{5}$ Department of Paediatrics, University of Melbourne, Parkville, Australia

Repeat expansions (RE) cause over 20 neurogenetic disorders of major clinical significance, which can present with heterogenous, overlapping clinical phenotypes. Ataxias are the most common of these including spinocerebellar ataxias (SCAs) 1, 2, 3, 6, 7, 8, 10, 12, 17, 36 and Friedreich ataxia. RE also underlie FragileX syndrome and Huntington disease. Single gene or small panel PCRbased methods are currently employed for diagnosis of SCAs, but can be slow and costly, with a current diagnostic yield of $\sim 20 \%$. To date, technical issues have prevented identification of RE in whole exome or whole genome sequencing (WES and WGS) datasets. Therefore, we developed a software tool called exSTRa (expanded STR algorithm) to identify RE using either WES or WGS. To validate exSTRa we analyzed cohorts of individuals with eight different known RE disorders ( $n=19$ affected individuals). Results were assessed by comparing to the known disease status determined by standard practice diagnostic tools. Repeat expansions were successfully identified in seven of eight disorders, with very high predictive capabilities (median area under the curve (AUC) of 0.9) and a median specificity and sensitivity of 0.99 and 0.75 respectively. These results were achieved regardless of whether the library preparation was PCR-free or not. A single affordable front-line test that is able to comprehensively detect the genetic basis of human disease is the ultimate goal of diagnostics for genomic medicine. Our method represents a significant step forward in fully exploiting the clinical utility of NGS datasets and facilitating the cost-effective implementation of precision medicine.

\section{ASDG Oral 10 \\ DNA QUALITATIVE AND QUANTITATIVE ASSESSMENT OF FFPE DNA IMPROVES THE SUCCESS OF HIGH-THROUGHPUT VARIANT PROFILING \\ Wei J', Kavanagh $C^{\prime}$, Kraitsek $S^{\prime}$, Qian J', Yu B ${ }^{1,2}$ \\ ${ }^{1}$ Department of Medical Genomics, Royal Prince Alfred Hospital, Camperdown, Australia \\ ${ }^{2}$ Sydney Medical School (Central), The University of Sydney, University of Sydney, Australia}

Genetic testing for somatic variants in cancers can help oncologists select effective targeted therapies for cancer patients. Recent advances in high-throughput technologies such as DNA mass spectrometry and massively parallel sequencing have enabled simultaneous testing of multiple clinically actionable targets that can enormously benefit critically ill cancer patients. However, DNA extracted from formalin-fixed, paraffin-embedded tissue (FFPE DNA) and plasma (circulating cell-free DNA; cfDNA) poses significant challenges for the success of these advanced multi-targeting assays, and therefore significantly limits their clinical utility. In order to prevent the prolonged turnaround time (TAT) due to assay failure, we validated and utilized the DNA mass spectrometry analysis (Agena SampleID panel) and a real-time quantitative PCR method (Alu qPCR) for DNA quality control (QC). For validation, a total of 130 DNA samples extracted from FFPE tissue, fresh blood and plasma were tested. We found a strong correlation between the amplifiable genomic copies per microliter obtained from these two assays (Pearson's $r=.82$ ). While Agena SampleID can provide an indication of multiplex amplification capacity of DNA template, Alu qPCR and data analysis can be completed within three hours with a high repeatability (average $\mathrm{CV}=11.9 \%$ ). Since implementation of the DNA QC, we have achieved a 3\% (30/946) DNA rejection rate and were able to maintain an average of $6.6 \pm 2.4$ days $(1 / 2017$ - 12/2017) TAT. Integration of the PCR based DNA assessments into a clinical diagnostic workflow provides a fast, robust and costeffective DNA quality control for downstream applications and minimizes TAT. 


\section{ASDG Oral 11 \\ MANAGING VARIANT REVIEW AND RECLASSIFICATION. RECOMMENDATIONS FROM THE AUSTRALIAN GENOMICS VARIANT RECLASSIFICATION WORKING GROUP.}

King $S^{1,2}$, Ingles $J^{5,6,7}$, Poplawski $N^{3,4}$, Bruno $D^{8}$, Tudini $E^{9}$, Spurdle $A^{9}$, Lunke $S^{8,21}$

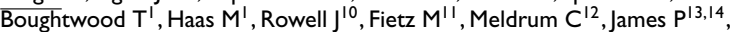
Bennetts $B^{6,15}$, Kirk $E^{16,17,18}$, McGaughran J ${ }^{19}$, Ronan $A^{20}$, Semsarian $C^{5,6,7}$, Stark $\mathrm{Z}^{1,8,21}$, Scott $\mathrm{H}^{1,2,22,23,24,25}$

${ }^{I}$ Australian Genomics Health Alliance, Melbourne, Australia

${ }^{2}$ Centre for Cancer Biology, SA Pathology \& UniSA Alliance, Adelaide, Australia

${ }^{3}$ Adult Genetics Unit, Royal Adelaide Hospital, Adelaide, Australia

${ }^{4}$ University Department of Paediatrics, University of Adelaide, Adelaide, Australia

${ }_{5}^{5}$ Molecular Cardiology Program, Centenary Institute, Sydney, Australia

${ }^{6}$ Sydney Medical School, University of Sydney, Sydney, Australia

${ }^{7}$ Department of Cardiology, Royal Prince Alfred Hospital, Camperdown, Australia

${ }^{8}$ Victorian Clinical Genetics Services, Murdoch Childrens Research Institute,

Parkville, Australia

${ }^{9}$ QIMR Berghofer Medical Research Institute, Brisbane, Australia

${ }_{10}$ Pathology Queensland, Royal Brisbane and Women's Hospital, Brisbane, Australia

${ }^{11}$ Department of Diagnostic Genomics, PathWest Laboratory Medicine WA, Nedlands,

Australia

${ }^{12}$ NSW Health Pathology, Newcastle, Australia

${ }^{13}$ Peter MacCallum Cancer Centre, East Melbourne, Australia

${ }_{14}$ Royal Melbourne Hospital, Parkville, Australia

${ }^{15}$ Department of Molecular Genetics, Western Sydney Genetics Program, The

Children's Hospital at Westmead, Sydney, Australia

${ }^{16}$ Randwick Genetics, NSW Health Pathology, Prince of Wales Hospital, Randwick, Australia

${ }^{17}$ Centre for Clinical Genetics, Sydney Children's Hospital, Randwick, Australia

${ }^{18}$ School of Women's and Children's Health, University of New South Wales, Sydney,

Australia

${ }^{19}$ Genetic Health Queensland, Royal Brisbane \& Women's Hospital, Brisbane,

Australia

${ }^{20}$ Hunter Genetics, Newcastle, Australia

${ }^{21}$ Department of Paediatrics, University of Melbourne, Melbourne, Australia

${ }^{22}$ School of Medicine, University of Adelaide, Adelaide, Australia

${ }^{23}$ Australian Cancer Research Foundation Cancer Genomics Facility, Centre for

Cancer Biology, SA Pathology, Adelaide, Australia

${ }^{24}$ School of Pharmacy and Medical Science, University of South Australia, Adelaide,

Australia

${ }^{25}$ Beat Cancer Principal Research Fellow, Adelaide, Australia

Background: The interpretation of the pathogenicity of a genetic variant is a challenging process wholly dependent on the standards and knowledge available at the time of reporting. While recent updates to guidelines have brought some standardization, changes to classifications are an inherent part of the interpretation process - due to differences in the implementation of guidelines, inequity in access to supporting evidence, updates to standards and the emergence of new data. Furthermore, variants have historically been over-interpreted, with discordance rates of $12-53 \%$ reported within clinical databases. Over 8000 disease gene variants have been classified in South Australia alone, indicating a substantial number of Australian reports may be inaccurate based on the discordance estimates from other databases. Methods: To address the challenge of managing changes to reported variants, the Australian Genomics Health Alliance established a Variant Reclassification Working Group. The group reviewed available literature and guidelines, and audited occurrences of variant reclassification and discordance within laboratories. Results: Instances of reclassification or discordance have never been systematically investigated in Australia. Identification of these events occurs on an ad hoc basis dependent on clinical demand, or via expert committee review and is substantially hampered by the lack of a national variant database. Conclusion: Management of variant review within current budgetary constraints is a significant challenge for healthcare providers. Improvements in 1) sharing of variant data and expert knowledge, 2) a national variant database, 3) partial automation of variant classification, and 4) clear guidance for discordance and notification processes are recommended.

\section{ASDG Oral 12 \\ WHOLE GENOME SEQUENCING IMPROVES GENETIC TESTING OUTCOMES IN HYPERTROPHIC CARDIOMYOPATHY}

Bagnall $R^{1,2}$, Ingles $J^{1,2,3}$, Dinger $M^{4,5}$, Cowley $M^{4,5}$, Barratt-Ross $S^{1,2}$, Minoche $A^{4}$, Lal $S^{2,3}$, Turner $C^{6}$, Colley $A^{7}$, Rajagopalan $S^{7}$, Berman $Y^{8}$, Ronan $A^{9,10}$, Fatkin $D^{11,12}$, Semsarian $C^{1,2,3}$

${ }^{1}$ Centenary Institute, Camperdown, Australia

Sydney Medical School, University of Sydney, Sydney, Australia

3 Department of Cardiology, Royal Prince Alfred Hospital, Sydney, Australia

${ }^{4}$ Garvan Institute of Medical Research, Sydney, Australia

${ }^{5}$ St Vincent's Hospital Clinical School, Sydney, Australia

6 The Sydney Children's Hospital, Westmead, Australia

${ }^{7}$ Department of Clinical Genetics, Liverpool, Australia

${ }^{8}$ Clinical Genetic Department, Royal North Shore Hospital, Sydney, Australia

${ }^{9}$ Hunter Genetics Unit, Newcastle, Australia

${ }^{10}$ University of Newcastle, Newcastle, Australia

${ }^{11}$ Victor Chang Cardiac Research Institute, Sydney, Australia

${ }^{12}$ Cardiology Department, St. Vincent's Hospital, Sydney, Australia

Background: Whole genome sequencing (WGS) can detect most types of genetic variants. Aims: We assessed WGS for hypertrophic cardiomyopathy (HCM) in which prior cardiomyopathy gene-panel sequencing, or exome sequencing, did not establish a molecular diagnosis, that is, gene-elusive HCM, and as a first-line genetic test Methods: WGS was performed on 46 gene-elusive HCM probands, 16 family members, and $12 \mathrm{HCM}$ probands with no prior genetic testing. We searched for coding-region variants and genomic rearrangements in 184 cardiac hypertrophy genes, deep intronic variants that alter RNA splicing, and mitochondrial genome variants. All splice-altering variants were validated using RNA extracted from fresh venous blood, or cardiomyocytes derived from induced pluripotent stem cells, of the patients. Results: We found a pathogenic or likely pathogenic variant in 10 out of $46(22 \%)$ gene-elusive HCM families. Four families had deep intronic variants in MYBPC3 that activate splicing of pseudo-exons; one family had a synonymous MYBPC3 variant shown to disrupt splicing; one family had mitochondrial genome variant; one family had a nonsynonymous MYH7 variant that was not genotyped during exome sequencing; and three families had variants in genes not included in prior cardiomyopathy gene-panel testing. As a first-line genetic test, WGS identified a pathogenic variant in 5 out of 12 (42\%) families with no prior genetic testing. Conclusions: WGS identified additional genetic causes of HCM over targeted gene sequencing approaches. Genetic screening of deep intronic regions identified pathogenic variants in $9 \%$ of gene-elusive HCM. These findings translate to more accurate diagnosis and management in HCM families.

\section{Concurrent Session 6 - Australasian Society of Inborn \\ Errors of Metabolism \\ ASIEM Oral 6 \\ EFFECTIVENESS OF EARLY HEMATOPOIETIC STEM CELL TRANSPLANTATION IN PREVENTING NEUROCOGNITIVE DECLINE IN MUCOPOLYSACCHARIDOSIS TYPE II}

\footnotetext{
Selvanathan $A^{1,2}$, Ellaway $C^{1,2,5}$, Wilson $C^{3}$, Owens $P^{1}$, Shaw $P^{2,4}$, Bhattacharya $\mathrm{K}^{1,2,5}$

${ }^{I}$ Genetic Metabolic Disorders Service, The Children's Hospital at Westmead, Sydney, Australia

${ }^{2}$ Discipline of Child and Adolescent Health, The University of Sydney, Sydney, Australia

${ }^{3}$ Starship Paediatric Metabolic Service, Starship Children's Hospital, Auckland, New Zealand

${ }^{4}$ Blood and Marrow Transplant Service, The Children's Hospital at Westmead, Sydney, Australia

${ }^{5}$ Discipline of Genetic Medicine, The University of Sydney, Sydney, Australia
}

The early progressive form of the X-linked disorder, Mucopolysaccharidosis Type II (MPS II), is characterized by cognitive decline and cardiorespiratory complications, which result in death before 20 years of age with considerable prior morbidity. MPS II occurs 
due to deficiency of the lysosomal enzyme, iduronate-2-sulfatase, which results in accumulation of the glycosaminoglycans, dermatan and heparan sulfate. Lifelong enzyme replacement therapy (ERT) is now the mainstay of treatment but is costly and ineffective in arresting cognitive decline. Other novel approaches, such as intrathecal ERT, come with significant risks. Hematopoietic stem cell transplantation (HSCT) appears to be effective in stabilising neurocognitive function if performed early, though data are limited. We aim to add Australasian data to this literature. We present a retrospective chart review of four patients who demonstrated neurocognitive stabilization with early HSCT. These patients represent all cases of MPS II who presented to two tertiary metabolic units between January 2012 and June 2014. The first three patients were transplanted prior to neurocognitive decline and are developing within the normal range. The first two are attending mainstream school without assistance, in stark contrast to the natural history of the disorder and those treated with ERT. The fourth patient, with pre-existing cognitive impairment, showed stabilization of disease. Our case series suggests that early HSCT is a potentially underutilized treatment strategy in MPS II. Further studies are necessary to delineate the appropriate age for transplantation, and identify genotypic factors that might affect suitability for transplant.

\section{ASIEM Oral 7 EFFECTIVENESS OF HEMATOPOIETIC STEM CELL TRANSPLANTATION IN PREVENTING NEUROCOGNITIVE DECLINE IN ASPARTYLGLUCOSAMINURIA: A CASE SERIES. \\ Selvanathan $A^{1,2}$, Wilcken $B^{1,2,5}$, Shaw $P^{2,3}$, Moore $F^{4}$, Bhattacharya $K^{1,2,5}$ \\ ${ }^{I}$ Genetic Metabolic Disorders Service, The Children's Hospital At Westmead, Sydney, Australia \\ ${ }^{2}$ Discipline of Child and Adolescent Health, The University of Sydney, Sydney, Australia \\ ${ }^{3}$ Blood and Marrow Transplant Service, The Children's Hospital at Westmead, Sydney, Australia \\ ${ }^{4}$ Department of Biochemical Genetics, The Children's Hospital at Westmead, Sydney, Australia \\ ${ }^{5}$ Discipline of Genetic Medicine, The University of Sydney, Sydney, Australia}

Aspartylglucosaminuria (AGU) is a recessively-inherited lysosomal storage disorder (LSD) affecting glycoprotein catabolism. The disease process is characterized by an initial period of normal development in infancy, a plateau in childhood and subsequent regression in adolescence and adulthood. No enzyme replacement therapy or other curative treatments are currently available, leading to a protracted period of significant disability prior to early death. Hematopoietic stem cell transplantation (HSCT) has demonstrated efficacy in other LSDs, by providing enzyme replacement therapy in both the viscera and the central nervous system. We hypothesize that HSCT may be similarly efficacious in AGU, but there are few and conflicting reports in the literature. We present a case series of three children with AGU, who represent all cases of AGU diagnosed in New South Wales since 2000. They received HSCT at different ages ( 9 years, 5 years and 5 months of age), with longterm follow-up post-transplant (over 7 years). Data were collected by retrospective review of medical records. The first two cases, who underwent HSCT after presenting with developmental delay, have ongoing moderate intellectual disability that appears to have stabilized (based on formal developmental assessments). The third case, transplanted prior to the onset of neurocognitive involvement, is developing normally. Complications of HSCT (including febrile neutropenia and graft-versus-host disease) occurred in all patients, but were treated appropriately and resolved. Our cases suggest that early HSCT may have a therapeutic role in treatment of AGU. Larger case series are needed to confirm this potential benefit.

\section{ASIEM Oral 8 \\ NSW EXPERIENCE WITH CASES OF ISOVALERIC ACIDEMIA}

AlHafid N', Devanapalli B', Wiley $\mathrm{V}^{1,2,3}$

${ }^{1}$ NSW Biochemical Genetics Service, Westmead, Australia

${ }^{2}$ NSW Newborn Screening Programme, Westmead, Australia

${ }^{3}$ University of Sydney, Sydney, Australia

Isovaleric acidemia (IVA) is an autosomal recessive disorder caused by a deficiency in isovaleryl-CoA dehydrogenase (IVD). The enzyme is responsible for catabolising isovaleryl-CoA, the third enzyme in the leucine catabolic pathway. A deficiency in IVD leads to the accumulation of isovalerylglycine in urine and isovalerylcarnitine in plasma. The clinical manifestations in the neonatal period include vomiting, severe acidosis, and characteristic odour of 'sweaty feet', lethargy, seizures and death if treatment is not initiated. The chronic intermittent form however, is associated with developmental delay with or without recurrent acidotic episodes during catabolic stress. Here we present 4 cases of IVA diagnosed in NSW during the neonatal period. Case 1: an infant male admitted on day 5 of life whose IVA treatment was complicated by intra-cerebellar hemorrhage causing him severe neurological damage. Case 2: A female infant who was asymptomatic identified by elevated $\mathrm{C} 5$ in the newborn screening dried blood spot (DBS) sample. Case 3 and 4: Term female and male infants respectively, admitted for poor feeding and lethargy, both responded well to therapy with no major life threatening events thereafter. All cases were identified through the Newborn Screening Program showing elevated C5 in their DBS sample although case 1 and 4 presented clinically before results were available. These cases show the variability in clinical manifestation of IVA even within the neonatal period highlighting the importance of the general health and wellbeing of the patients for a better outcome for IVA treatment.

\section{ASIEM Oral 9 \\ BEDSIDE TEST FOR DEFICIENT PERIPHERAL WARMTH PERCEPTION: A COMMON AND EARLY SIGN IN FABRY DISEASE}

Nedanovski B', Talbot A', Nicholls $\mathrm{K}^{1,2}$

${ }^{I}$ The Royal Melbourne Hospital, Melbourne, Australia

${ }^{2}$ The Royal Melbourne Hospital Department of Nephrology, Melbourne, Australia

Background: Fabry disease classically presents in childhood with acroparesthesia and heat intolerance. Time to diagnosis can take up to 20 years. Increased warmth detection threshold is often overlooked, with course and response to enzyme replacement therapy (ERT) poorly documented. Aims: To determine: (1) prevalence and severity of sensory neuropathy in Fabry patients using simple bedside examination; (2) effect of ERT. Method: Patients undergo serial neurological examination 6-12 monthly, comprising lower and upper limb (LL/UL) stimuli (heat probe/ cotton wool/ pin prick), touched to the knee/elbow as reference, then to distal extremities and moved proximally until perceived as equal to the reference. Analgesia level was arbitrarily scored: none $=0$, toetips/fingertips $=1$, interphalangeal joint toe/DIPJ $=2 \ldots$ knee/elbow $=10$. Sealed water containers provided heat (70 degrees Celsius); $t$ testing was used for analysis. Results: In 118 patients (68 females) at baseline, symmetrical LL deficit in warmth perception was commoner than light touch and pain ( $89 \%$ vs. $38 \%$ vs. $33 \%$ ). Seven patients $<20$ years demonstrated a similar pattern ( $86 \%$ vs $29 \%$ vs $0 \%)$. UL deficits were similar $(79.7 \%$ vs $38 \%$ vs. $31 \%)$.Deficit severity (mean+/-SEM) was (LL $4.1 \pm 0.3$ vs. $0.96 \pm 0.2$ vs. $1.4 \pm 0.2$, UL $2.4 \pm 0.2$ vs. $0.9 \pm 0.2$ vs. $1.1 \pm 0.2, p<.0001$ ). Over time, in 70 untreated patients ( $51 \mathrm{fe}-$ males), deficit in warmth perception progressed in all limbs $(p<.01$ at 5-10 years and $>10$ years). In 24 patients ( 20 males) on ERT, LL deficits improved at $0.5-1$ years $(p=.01)$ and did not progress thereafter. Conclusion: Heat perception deficit is common, often isolated, 
increases over time and responds to ERT. Bedside testing may flag young patients for Fabry screening.

\section{Concurrent Session 7 - Australasian Association of Clinical Geneticists \\ AACG Oral 7 \\ TELLING THE UNTOLD STORY: THE ECONOMIC AND PSYCHOSOCIAL IMPACTS ON FAMILIES AFFECTED BY INTELLECTUAL DISABILITY}

Schofield $D^{1}$, Tan $\mathrm{O}^{\prime}$, Rajkumar $\mathrm{R}^{2,3}$, Kasparian $\mathrm{N}^{4,5}$, Shrestha $\mathrm{R}^{1}$, Boyle $\mathrm{J}^{6}$, Rice $M^{1}$, Christie $L^{6}$, Leffler $M^{6}$, Murray $L^{6}$, West $S^{1}$, Roscioli $T^{4,7,8}$, Field $M^{6,9}$

${ }^{I}$ GenIMPACT, Department of Economics, Faculty of Business and Economics,

Macquarie University, Sydney, Australia

${ }^{2}$ Genome.One, Darlinghurst, Australia

${ }^{3}$ Kinghorn Centre for Clinical Genomics, Darlinghurst, Australia

${ }^{4}$ UNSW Medicine, Kensington, Australia

${ }^{5}$ Heart Centre for Children, The Sydney Children's Hospitals Network, Randwick and Westmead, Australia

${ }^{6}$ Genetics of Learning Disability (GOLD) service, Newcastle, Australia

${ }^{7}$ Sydney Children's Hospital, Randwick, Australia

${ }^{8}$ Neuroscience Research Australia, Randwick, Australia

${ }^{9}$ Department of Clinical Genetics, Royal North Shore Hospital, St Leonards, Australia

Purpose: Data on the financial and psychosocial costs incurred by families affected by intellectual disability (ID) in Australia is scarce, thus limiting the capacity to value the benefits of effectively diagnosing and preventing ID through the application of molecular diagnosis, including genomic testing. We report on the first large, indepth study exploring the economic, psychosocial and potential reproductive impacts of a molecular diagnosis for moderate to severe ID. Methods: To date we have surveyed 50 families from the Genetics of Learning Disability service and Liverpool Hospital, Sydney. A survey instrument was developed specifically for this study to assess quality of life and a range of psychosocial factors, income, welfare, savings and assets, education and employment, living arrangements, transport subsidies and family out-of-pocket costs. Results: From our preliminary data, we estimated the combined costs to Commonwealth, state, and private households total $\$ 13.9$ million per household up to the age of 48 years. The cost burden on these families is $\$ 5.8$ million per household, mainly due to lost income and outof-pocket expenses. Cost to the Commonwealth totalled \$2.4 million per household and cost to the state government totalled $\$ 5.7$ million per household. The families are also under significant psychosocial strain, with the majority of carers reporting levels of depression, anxiety, and stress indicative of a need for clinical intervention. Conclusion: Our study shows that families affected by ID experience significant burden. This data can be used to benchmark the potential benefits of precision medicine and informed reproductive decision making.

\section{AACG Oral 8}

\section{RECESSIVE SPONDYLOCARPOTARSAL SYNDROME DUE TO} COMPOUND HETEROZYGOSITY FOR VARIANTS IN MYH3

Robertson $S^{\prime}$, Jenkins $Z^{\prime}$, Wells $C^{\prime}$, Cameron-Christie $S^{\prime}$, Wei $W^{\prime}$, Tang $C^{\prime}$, Simon $M^{2,3}$, Wessels $M^{3}$, Takei $R^{1}$, Arts-Tesselaar $C^{4}$, Sandaradura $S^{5}$, Sillence $D^{5}$, Cordier $\mathrm{M}^{6}$, Veenstra-Knol $\mathrm{H}^{7}$, Trevisson $\mathrm{E}^{8}$, Markie $\mathrm{D}^{1}$

${ }^{I}$ University of Otago, New Zealand

${ }^{2}$ University Medical Center Utrecht, Utrecht, The Netherlands

${ }^{3}$ Erasmus University, Rotterdam, The Netherlands

${ }^{4}$ Amphia Hospital, Breda, The Netherlands

${ }^{5}$ Children's Hospital at Westmead, Sydney, Australia

${ }^{6}$ Hôpitaux de Lyon, Lyon, France

${ }^{7}$ University of Groningen, Groningen, The Netherlands

${ }^{8}$ University of Padova, Padova, The Netherlands

Spondylocarpotarsal syndrome (SCTS) is characterized by vertebral fusions and fusion of the carpal and tarsal bones. Bi-allelic mutations in FLNB cause SCTS in some families, while monoallelic variants in the gene encoding embryonic heavy chain myosin 3 , MYH3, have been implicated in dominantly inherited forms. Here five FLNB mutation-negative cases from three families were hy- pothesized to have a recessive form of SCTS on account of sib recurrence. Whole Exome Sequencing (WES) showed that all five were heterozygous for one of two splice-site variants in MYH3. Despite 3 of the 5 cases sharing two allelic haplotypes over MYH3, no second variant could be identified by WES. Subsequent WGS demonstrated a variant altering a conserved splice donor site in the 5'UTR of MYH3, a region of the gene not captured by exome capture platforms. Expanding the case cohort to 16 unsolved SCTS cases, 9 had truncating mutations transmitted by unaffected parents, with 6 inheriting the same 5'UTR variant in trans, an observation at variance with the allele frequency for this variant in population databases. This variant disrupts splicing of exons $1-3$ in the 5' UTR but is still permissive of MYH3 translational initiation, albeit with reduced efficiency. Although some MYH3 variants cause dominant SCTS, these data indicate that many truncating variants do not lead to the disease except when in trans with a second hypomorphic allele. These observations make genetic diagnosis and counseling challenging in the context of simplex presentations of SCTS especially using a genotype-first methodology.

\section{AACG Oral 9 \\ THE FIRST 500 DIAGNOSTIC EXOMES: A DEMONSTRATION OF SAFETY, CLINICAL UTILITY, TRANSLATION AND COST-EFFECTIVENESS}

Roscioli $T^{1,3,11}$, Cliffe $C^{1}$, Elakis $G^{1}$, Zhu $Y^{1,2}$, Nixon $C^{1,3}$, Mullan $G^{\prime}$, Lang $S^{\prime}$, Richards $A^{\prime}$, Quayum $N^{1}$, Ades $L^{4,5}$, Amor $D^{6}$, Bakshi $M^{7}$, Berman $Y^{8}$, Brown $N^{9}$, Chung $C^{7}$, Colley $A^{7}$, Collins $F^{10}$, Edwards $M^{7}$, Ellaway $C^{5,11}$, Ewans $L^{10,13,14}$, Fahey $M^{15}$, Field $M^{2,8}$, Freckmann $M^{8}$, Gabbett $M^{16,17}$, Goel $H^{18}$, Ghedia $S^{8}$, Goodwin $L^{19}$, Hackett $A^{2,8}$, Hunter $M^{15}$, Jones $K^{4,5}$, Josephi-Taylor $S^{4,5}$, Kamian $B^{20}$, Kennedy $D^{1,21}, M a A^{4,5}$, McGillivray $G^{11}$, Mowat $D^{11,12}$, Palmer $E^{2}$, Pinner $J^{11,12}$, Rajagopalan $S^{7}$, Ronan $A^{18}$, Sachdev $R^{11,12}$, Sandaradura $S^{4,5}$, Sinnerbrink $I^{19}$, Smith $J^{4,5}$, Turner $A^{11,12}$, Walsh $M^{23}$, Wallis $M^{9}$, Wilson $M^{4,5}$, Worgan $L^{10}$, Schofield $D^{24}$, Lau $C^{\prime}$, Kirk $E^{1,11,12}$, Mead $S^{\prime}$, Buckley $M^{\prime}$

${ }^{1}$ Sydney Children's Hospital, Sydney, Australia

2 The Genetics of Learning Disability Service, Waratah, Australia

${ }^{3}$ Neuroscience Research Australia and Prince of Wales Clinical School, University of New South Wales, Kensington, Australia

${ }^{4}$ Department of Clinical Genetics, Children's Hospital at Westmead, Sydney, Australia ${ }^{5}$ Disciplines of Child and Adolescent Health and Genetic Medicine, University of Sydney, Newtown, Australia

${ }^{6}$ The Royal Children's Hospital and Victorian Clinical Genetics Service, Flemington, Australia

${ }^{7}$ Clinical Genetics Department, Liverpool Hospital, Liverpool, Australia

${ }^{8}$ Department of Clinical Genetics, Royal North Shore Hospital, St Leonards, Australia

${ }^{9}$ Clinical Genetics Service, Austin Hospital, Melbourne, Australia

${ }^{10}$ Department of Medical Genomics, Royal Prince Alfred Hospital, Newtown, Australia

${ }^{11}$ Centre for Clinical Genetics, Sydney Children's Hospital, Randwick, Australia

12 School of Women's and Children's Health, University of New South Wales,

Kensington, Australia

${ }^{13}$ St Vincent's Clinical School, University of New South Wales, Darlinghurst, Australia

${ }^{14}$ Kinghorn Centre for Clinical Genomics, Garvan Institute of Medical Research, Darlinghurst, Australia

${ }^{15}$ Monash Genetics and Monash Health and Department of Paediatrics, Monash

University, Melbourne, Australia

${ }_{17}^{16}$ Queensland University of Technology, Brisbane, Australia

${ }_{17}$ Genetic Health Services Queensland, Brisbane, Australia

${ }^{18}$ Hunter Genetics, Newcastle, Australia

${ }^{19}$ Genetics Services, Nepean Hospital, Nepean, Australia

${ }^{20}$ Genetic Services of Western Australia, Perth, Australia

${ }^{21}$ Mothersafe, Royal Hospital for Women, Randwick, Australia

${ }^{22}$ The Mercy Hospital, Melbourne, Australia

${ }^{23}$ Genetic Medicine and Familial Cancer Centre, the Royal Melbourne Hospital,

Flemington, Australia

${ }^{24}$ GenIMPACT: Centre for Economic Impacts of Genomic Medicine, Faculty of Business \& Economics, Macquarie University, Epping, Australia

Purpose: Whole exome sequencing (WES) is rapidly becoming the standard of care for genetic services. We present the results of 500 clinical exomes performed at the Randwick Genetic Laboratory. Methods: WES was performed in 204 probands with suspected Mendelian disorders, together with family members. Ampliseq RDY exome, libraries were analyzed on a Life Technologies Proton instrument. Data were analyzed using an in house pipeline with variant reporting following ACMG guidelines. Results: WES resulted in greater than $43 \%$ definitive findings with a small number of variants of uncertain significance. The diagnostic rate has 
increased over time, likely reflecting refinements in clinician referral practice as well as improved functionality of bioinformatics pipelines. Two families (1\%) with Cantu and MoyaMoya syndromes had results which could lead to pharmacologic interventions. 33 families $(16 \%)$ had de novo variants and seven $(3.5 \%)$ were X-linked with significant implications for recurrence risk. Two prenatal diagnoses have been performed based on WES results. A diagnosis was made in $6 / 8(75 \%)$ rapid turnaround studies, including two during pregnancy. Almost $10 \%$ of the diagnoses were unanticipated by the clinical teams and involved significant changes in diagnostic category. Four likely novel genes involved in known biological pathways were identified, enabling research participation. Conclusions: The minimum number of management changing outcomes was at least $20 \%$ within the 12 month period of this study. The number of secondary findings was small at about $1 \%$. This demonstrates that WES is characterized by high levels of patient safety and clinical utility with likely cost effectiveness.

\section{AACG Oral 10 \\ WHOLE EXOME SEQUENCING IN INFANTS WITH CONGENITAL HEARING LOSS}

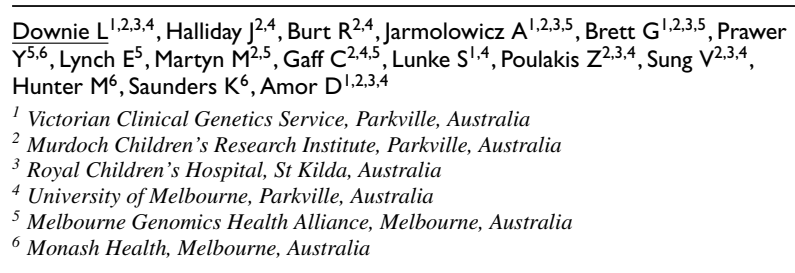

Background: The Melbourne Genomics Health Alliance is establishing systems and producing evidence to guide incorporation of genomics into the Victorian healthcare system. Through one of its clinical flagship projects, the Alliance is offering WES to families who have an infant with moderate to profound bilateral hearing loss born in 2016 or 2017. Aim: The aim of this project is to define the genetic etiology of congenital hearing loss, streamline the care of affected children and explore parents' interest in genomic testing. Methods: Families who have an eligible child are identified by the statewide Victorian Infant Hearing Screening Program (VIHSP). Participants are offered pediatrician-run hearing clinic appointment and genetics appointment. WES with targeted gene analysis is performed in conjunction with microarray. Results: $70 \%$ of eligible participants consented to WES. Of the 107 patients recruited, 52 results have been issued to date. The rate of diagnosis is $67 \% ; 33 \%$ have connexin mutations, $17 \%$ have another non-syndromic deafness gene identified, $17 \%$ have a syndromic diagnosis. Microarray has contributed to $10 \%$ of diagnoses when combined with WES results. An additional $34 \%$ of participants have received a diagnosis using WES over our centre's usual investigation methods of connexin testing and microarray. Discussion: Understanding the genetic etiology for infants with congenital hearing loss provides important information for families regarding prognosis, reproductive risk and screening for associated health problems in their child. Conclusion: This study provides a comprehensive understanding of the genetic etiology of congenital hearing loss over this two year period.

\section{AACG Oral 11 \\ A RETROSPECTIVE REVIEW OF A MULTIDISCIPLINARY HEARING LOSS CLINIC: 2015-2017}

Ho $\mathrm{H}^{2}$, Springer $\mathrm{A}^{1,2}$, Hunter $\mathrm{M}^{1,2}$

${ }^{1}$ Monash Genetics, Melbourne, Australia

${ }^{2}$ Department of Paediatrics, Monash University, Melbourne, Australia

Background: We established a multidisciplinary pediatric genetic hearing loss clinic for considering genetic diagnoses in children and offering further genetic testing for a subset. Patients were prescreened for GJB2, microarray, imaging, TFT, CMV and hematuria/proteinuria prior to genetics assessment. Funded genetic testing was routinely offered in cases where there was a family history, management or pregnancy implications, or patients could self-fund. Method: We performed a retrospective review to assess rates of genetic testing and diagnosis from 2015-2017. Outpatient records were reviewed and data, including referral source, ethnicity, family history, inheritance pattern, phenotype, genetic testing, and diagnosis were extracted and analyzed for individuals seen in the clinic during 2015-2017. Results: A total of 90 patients were seen, 22 received a genetic diagnosis and 7 a likely genetic diagnosis $(32 \%)$. Of these 90 , gene panel or exome sequencing was performed in 42 and a genetic diagnosis was confirmed or likely in 17 (40\%). Cascade testing was performed in relatives of 17 probands, and demonstrated that 13 cases were inherited. 25 patients had syndromic or likely syndromic causes. Discussion: This review reports on real world assessment and selection for genetic testing in hearing loss. Clinical prioritization for genetic testing to maximize clinical utility is appropriate within the public healthcare setting and the application of methods that efficiently optimize outcomes for patients effectively benefit the healthcare system. Our diagnosis rate is higher than previously reported similar studies and demonstrates the clinical utility of NGS testing in a carefully selected subset of patients.

\section{AACG Oral 12 \\ TUBB6 ASSOCIATED WITH A NEW PHENOTYPE OF NONPROGRESSIVE CONGENITAL FACIAL PALSY, BILATERAL PTOSIS, VELOPHARYNGEAL DYSFUNCTION.}

Fazeli $W^{1,2,3}$, Herkenrath $P^{\prime}$, Stiller $B^{4}$, Neugebauer $A^{5}$, Fricke $J^{5}$, Lang-Roth $R^{6}$, Nürnberg $G^{7}$, Thoenes $M^{8}$, Becker $J^{8}$, Altmüller $J^{7,8}$, Volk $A^{9}$, Kubisch $C^{9}$, Heller $\mathrm{R}^{8}$

${ }^{1}$ Department of Paediatrics, University Hospital Cologne, Cologne, Germany ${ }^{2}$ Institute for Molecular \& Behavioral Neurosciences, University of Cologne, Cologne, Germany

${ }^{3}$ German Centre for Neurodegenerative Diseases (DZNE), Bonn, Germany

${ }^{4}$ Department of Cardiovasc. Diseases, German Heart Centre, Technical University Munich, Munich, Germany

${ }_{5}^{5}$ Department of Ophthalmology, University Hospital Cologne, Cologne, Germany

${ }^{6}$ Department of Otorhinolaryngology, University Hospital Cologne, Cologne, Germany ${ }^{7}$ Cologne Centre for Genomics (CCG), University Hospital Cologne, Cologne. Germany

${ }^{8}$ Institute of Human Genetics, University Hospital Cologne, Cologne, Germany ${ }^{9}$ Institute of Human Genetics, University Medical Centre Hamburg-Eppendorf (UKE), Hamburg, Germany

Introduction: Congenital cranial dysinnervation disorders (CCDD) are a genetically and clinically heterogeneous group of disorders affecting the innervation of specific occulo-cranio-facial muscles. Non-progressive isolated CCDD are in some cases allelic to complex disorders of brain development as illustrated by the example of TUBB3 where specific heterozygous mutations may cause isolated congenital fibrosis of extraocular muscles (CFEOM) type 3A or cortical dysplasia, complex, with other brain malformations (CDCBM) type 1. Methods and Results: Using linkage analysis and DNA sequencing, we studied a 5-generation pedigree with autosomaldominant inheritance of a new phenotype consisting of facial paresis, ptosis and velopharyngeal dysfunction with rhinophonia. A heterozygous TUBB6 variant strictly co-seqregated with the phenotype and affected an amino residue that is highly conserved in evolution. Exclusion of pathogenic variants in other genes through whole exome sequencing, expression data for TUBB 6, 3D molecular modelling, and functional experiments in yeast all supported the interpretation that the TUBB6 variant c. $1181 \mathrm{~T}>\mathrm{C}$ is indeed the causative mutation. TUBB6 mutation analysis of additional patients with clinically suspected CCDD did not reveal any additional mutations in this gene. Conclusion: This is the 1st report implicating TUBB6 in 
human disease. A definitive proof that the above described phenotype is a CCDD in the sense of a neurodevelopmental disorder is still lacking. Our study adds to the genetic heterogeneity of tubulinopathies. Further imaging and functional work is planned to understand the mechanisms of TUBB6 action.

\section{Concurrent Session 8 - Australasian Society of Genetic Counsellors \\ ASGC Oral 7 \\ 'IT'S GOOD TO TAKE IT IN STAGES': MAPPING AUSTRALIAN GENOMICS EDUCATION AND DEVELOPING EVIDENCE-BASED TOOLS}

Nisselle $A^{1,2,3}$, Martyn $M^{2,3,4}$, Jordan $\mathrm{H}^{3}$, Dunlop $K^{1,5}$, Terrill $B^{1,6,7}$, Metcalfe $S^{1,2,3}$, Gaff $C^{1,3,4,8}$

${ }^{1}$ Australian Genomics Health Alliance, Parkville, Australia

${ }^{2}$ Murdoch Children's Research Institute, Parkville, Australia

${ }^{3}$ The University of Melbourne, Parkville, Australia

${ }^{4}$ Melbourne Genomics Health Alliance, Parkville, Australia

${ }^{5}$ Centre for Genetics Education, NSW Health. St Leonards, Australia

${ }^{6}$ Kinghorn Centre for Clinical Genomics, Garvan Institute of Medical Research

Darlinghurst, Australia

${ }^{7}$ Genome.One, Sydney, Australia

${ }^{8}$ Walter and Eliza Hall Institute, Parkville, Australia

Background/Aim: Implementing genomic medicine successfully into healthcare requires relevant education/training strategies, underpinned by research. Aims of this Australian Genomics Health Alliance project were to: 1) map clinical genomics education (GE) in Australia; 2) design a flexible genomics education evaluation framework; and 3) establish a network to test the framework and create an evidence base to guide appropriate, high-quality education. Methods: Current clinical GE activities were mapped via desktop audit plus semi-structured interviews with education providers. Transcribed interviews underwent content and thematic analysis. A draft evaluation framework was developed using literature and interview data, and refined at a workshop of international genomics education/evaluation experts. Participants agreed to test the framework in local contexts. Results: Mapping revealed 20 university postgraduate courses/subjects, 37 continuing professional development programs, and 2 massively open online courses. Interviews $(n=32)$ revealed that few activities were based in formal education/evaluation theories. Providers felt non-genetic health professionals need basic understanding of genomics to communicate, make test decisions and interpret results, supported by guidelines and specialist geneticists as required. The draft evaluation framework spans planning, development, delivery and outcomes. Twenty-four experts from Australia, the UK, USA and Canada refined the framework, with use-case testing continuing and formal network establishment at HGSA ASM 2018. Discussion/Conclusion: The mapping methodology allows future rigorous data collection and reporting as new programs emerge. Currently, there are many ad hoc clinical GE programs, however the evaluation framework and network create an opportunity for a more cohesive national approach to clinical genomics education, with potential for international applications.

\section{ASGC Oral 8 \\ 'A LEAP OF FAITH': EXPLORING PERSONAL GENOMIC TESTING FOR NUTRITION AND WELLNESS IN AUSTRALIA}

Tutty $\mathrm{E}^{1,2}$, Hickerton $\mathrm{C}^{1,2}$, Adamski $\mathrm{M}^{3}$, Savard $\mathrm{J}^{4,5,6}$, Terrill $\mathrm{B}^{6,7,8}$, Newson $\mathrm{A}^{5,6}$, Wilson $B^{9}$, Gaff $C^{10}$, Gray K ${ }^{2}$, Middleton $A^{\prime \prime}$, Metcalfe $S^{1,2}$

${ }^{1}$ Murdoch Children's Research Institute, Australia

${ }^{2}$ The University of Melbourne, Australia

${ }^{3}$ Monash University, Australia

${ }^{4}$ Deakin University, Australia

${ }^{5}$ The University of Sydney, Australia

${ }^{6}$ Garvan Institute of Medical Research, Australia

${ }^{7}$ The University of New South Wales, Australia

${ }^{8}$ Genome.One, Australia

${ }^{9}$ The University of Ottowa, Canada

${ }^{10}$ Melbourne Genomics Health Alliance, Australia

${ }^{11}$ Society and Ethics Research, Connecting Science, Wellcome Genome Campus, England

The emerging science of nutritional genomics informs personal genomic testing for nutrition and wellness (PGT-NG); however, many tests lack sufficient clinical validity and utility. Nevertheless, testing is increasingly available to Australians and marketed online by complementary/alternative medicine (CAM) providers. Little is known about information and support given to consumers. This study explored how PGT-NG is framed online to the Australian public, and experiences of Australians who have pursued PGT-NG. Websites offering testing were assessed using a novel framework incorporating informational content and marketing approaches. Online survey respondents from the Genioz (Genomics: National Insights of Australians) study participated in semi-structured interviews. Data were co-coded and analyzed thematically. Thirty-nine websites were assessed (4 PGT-NG companies and 35 CAM providers). Content was emotive and lacked necessary information for informed decisionmaking. All 12 interview participants had testing with a CAM provider. Unsure what to expect from results, participants took 'a leap of faith' that PGT-NG would 'solve' their chronic health problems. Although most felt translating results into real-life actions was difficult, a positive practitioner relationship mitigated disappointment. Participants believed MTHFR gene testing was particularly empowering. Websites framed PGT-NG as a tool to optimize health; claims appeared to give hope to the chronically unwell, but may produce disappointment in the well population. Findings highlight the fast-growing trend of PGT-NG being offered through CAM providers. Disseminating transparent information is essential for Australians to be supported regarding their decisions about PGTNG.

\section{ASGC Oral 9 \\ WORKING WITH COMMUNITIES: ENGAGING THE PUBLIC ABOUT THE DECISION TO PURSUE PERSONAL GENOMIC TESTING}

Hickerton $C^{1,2}$, Savard $\mathrm{J}^{3,4,5}$, Metcalfe $S^{1,2}$, Terrill $B^{5,6,7}$, Newson $A^{4,5}$, Gaff $C^{8}$,

Gray $\mathrm{K}^{2}$, Middleton $\mathrm{A}^{9}$, Wilson $\mathrm{B}^{10}$

${ }^{1}$ Murdoch Children's Research Institute, Melbourne, Australia

${ }^{2}$ The University of Melbourne, Melbourne, Australia

${ }^{3}$ Deakin University, Geelong, Australia

${ }^{4}$ The University of Sydney, Sydney, Australia

${ }^{5}$ Garvan Institute of Medical Research, Sydney, Australia

${ }^{6}$ The University of New South Wales, Sydney, Australia

${ }^{7}$ Genome.One, Sydney, Australia

${ }^{8}$ Melbourne Genomics Health Alliance, Melbourne, Australia

${ }^{9}$ Society and Ethics Research, Connecting Science, Wellcome Genome Campus,

Cambrigde, England

${ }^{10}$ The University of Ottawa, Ottawa, Canada

Personal genomic testing (PGT) for multiple purposes is marketed to ostensibly healthy people. Interest in these tests has significantly expanded in recent years, blurring boundaries between medical, 'wellness' and 'recreational' genomic testing. As part of our Genioz project, in 2017 we conducted nine public deliberative workshops Australia-wide, involving 129 participants with $(n=23)$ and 
without $(n=106)$ experience of testing. We aimed to explore nonexpert perspectives on PGT. Drawing on earlier survey and interview data, we selected three categories of testing for exploration: ancestry $(n=61)$, wellness $(n=34)$, and children's health and aptitudes $(n=34)$. Four stepwise, neutrally-framed information sets PGT, with time for facilitated discussion. Data were captured using a mixed methods approach. We report the results of: tracker questions from 5 time points in the workshops (including baseline); participant attitudes regarding information consumers should know about PGT; and a thematic analysis of discussions and written responses by participants. Tracker question data during the workshops indicate that participants became less comfortable with, and more thoughtful about, pursuing testing. Discussions and written responses reflected this change, with participants identifying ethically complex issues (about consent, family communication and privacy) that aligned with information they rated as important to know when considering PGT. This deliberative methodology highlights a useful public engagement strategy, demonstrating that non-experts can contribute to meaningful conversations about the decision to pursue PGT. These findings will inform policy and resources to help people critically reflect so they can make informed decisions around PGT. were presented pertaining to key scientific and ethical issues in

\section{ASGC Oral 11 \\ A NOVEL APPROACH FOR OFFERING ADDITIONAL FINDINGS TO PATIENTS: SEPARATING THIS DECISION FROM DIAGNOSTIC TESTING}

Lynch $E^{1,2,3}$, Martyn $M^{1,3,4}$, Kanga-Parabia $A^{1,3,4}$, James $P^{5,6}$, Lunke $S^{2,3}$, Macciocca $\mathrm{I}^{2,3}$, Wallis $M^{4,7}$, Hunter $M^{8,9}$, Trainer $A^{5,6}$, Halliday $\mathrm{J}^{3,4}$, Brown $\mathrm{N}^{2,7}$, Keogh $\mathrm{L}^{4}$, Wale J', Winship $\mathrm{I}^{4,5}$, Bogwitz $\mathrm{M}^{5,6}$, Winship $\mathrm{I}^{1,4}$, Melbourne Genomics Health Alliance ${ }^{\prime}$, Gaff $\mathrm{C}^{1,4}$

${ }^{1}$ Melbourne Genomics Health Alliance, Parkville, Australia

${ }^{2}$ Victorian Clinical Genetics Service, Parkville, Australia

${ }^{3}$ Murdoch Children's Research Institute, Parkville, Australia

${ }^{4}$ University of Melbourne, Parkville, Australia

${ }^{5}$ The Royal Melbourne Hospital, Parkville, Australia

${ }^{6}$ Peter MacCallum Cancer Institute, Parkville, Australia

${ }^{7}$ Austin Health, Heidelberg, Australia

${ }^{8}$ Monash Health, Clayton, Australia

${ }_{9}^{9}$ Department of Paediatrics, Monash University, Melbourne, Australia

Genomic sequencing has the potential to reveal information unrelated to diagnostic indications for testing, including the presence of variants that may predict future health risks. Several studies are generating evidence regarding uptake and management of additional findings (AFs) in clinical settings, with consent for AFs at the time of diagnostic testing and focus on health outcomes. We evaluate a novel model, offering reanalysis for AFs to adults after they have received genomic sequencing results for their clinical indication. Adults who received exome sequencing results are later recontacted to offer reanalysis of their stored data for AFs. Those interested receive decision support materials and genetic counseling before deciding whether to consent. Evaluation focusses on aspects impacting on how AFs are provided, including patient decision-making, uptake, clinical/laboratory service impact, psychosocial impact and views on future service delivery. To date, 57 patients have been approached, with 14 opting to attend for genetic counseling. Of the 6 who have attended, all decided to receive additional findings. 6/57 actively opted-out, citing logistical reasons, preference to address health problems as they arise or being advised by family members to decline. Of those approached to date, $83 \%$ had earlier expressed hypothetical interest in receiving AFs. Recruitment will cease by end 2018. Results from this novel model will provide Australian data to complement international studies and enable comparison with studies in healthy adults and pediatric cohorts.

\section{ASGC Oral 12 \\ IMPLEMENTATION OF A LABORATORY-BASED GENETIC COUNSELOR IN A TERTIARY PEDIATRIC HOSPITAL}

Curnow $L^{1,2,3}$, Smagarinsky $Y^{1,2,3}$, White $\mathrm{S}^{1,2}$, Savoia $\mathrm{H}^{3}$, Phillips $\mathrm{J}^{3}$, Macciocca $\mathrm{I}^{1,2}$

${ }^{I}$ Victorian Clinical Genetics Services, Melbourne, Australia

${ }^{2}$ Murdoch Children's Research Institute, Melbourne, Australia

${ }^{3}$ Royal Children's Hospital, Melbourne, Australia

lines or policies previously available, the default practice by most clinical laboratories has been to not return genomic data to individuals. Concerns have been raised about individuals having access to their data. There is emerging support for individuals to have access to their genomic data. Genome.One has developed and implemented a transparent policy on return of genomic data to patients and consumers. Process: (1) Review literature and current practices; (2) Review State, National and international guidelines examining access rights; (3) Legal review of draft policy including recommendations; (4) Establish processes for implementation; (5) Internal and external communication about policy and implementation; (6) Survey of client satisfaction with the protocol. Outcome: Genome.One patients have access to their genomic data on an encrypted hard drive including: (1) VCF, BAM and FASTQ file; (2) PDF copy of their pathology report; (3) Fact sheet outlining the contents of the hard drive, and providing guidance and information about reanalysis, supply of data to third parties, and disclaimers. To date 15 requests have been received and processed for release of genomic data, $7(47 \%)$ for transfer of data to their treating clinician, and 8 (53\%) for access to their data for personal use.
The Royal Children's Hospital ( $\mathrm{RCH}$ ) in Melbourne has seen a dramatic increase in genetic test requests and associated expenditure in recent years, without acompanying government funding. This ing in a 3-year grant to fund 2 laboratory-based genetic counselors (LBGCs). The aim of this appointment is to facilitate the development of processes that ensure access to quality, cost-effective, clinically indicated and appropriately targeted genetic and genomic testing. The effectiveness of LBGCs has been demonstrated in the USA, and at least one Australian hospital (RNS, NSW) has instituted a genetic stewardship program to address issues arising from increased demand for genetic testing, but to our knowledge, this is the first formally funded role in Australia. Since the role commenced, a major responsibility has been reviewing complex genetic test requests. We are providing an interface between the referring clinicians and the laboratory, ensuring appropriate clinical information is provided and adequate consent obtained where necessary. We source accredited laboratories with clinically acceptable turnaround times, which prompted a successful application to the RCH Foundation, result- 
enables clinicians to make informed decisions regarding patient care. We also plan to develop streamlined processes in conjunction with the electronic medical record system (EPIC) for genetic/genomic test ordering and consent, including decision support and accompanying education of medical specialists. At this preliminary stage in the implementation of the role at $\mathrm{RCH}$, we will present three cases that demonstrate the value of LBGCs at RCH and postulate the evolution of the workplace-model for LBGCs within an Australian health policy context.

\section{ASIEM Nurses/Allied Health Oral \\ CHALLENGES OF GLUTARIC ACIDEMIA (GA1) \\ MANAGEMENT - GUIDELINES, OUTCOMES AND \\ PRACTICALITIES}

Sweeney $\mathrm{A}^{\prime}$, Thompson $\mathrm{S}^{2}$

${ }^{I}$ Women's and Children's Hospital, Adelaide, Australia

${ }^{2}$ The Children's Hospital at Westmead, Sydney, Australia

Background: Australasian metabolic clinics have been managing NBS positive patients with glutaric acidemia type 1 (GA1) since 2002, prior to the publication of management guidelines based on European and U.S. cohorts. Management protocols have developed within clinics rather than Australasia wide and poor outcome is still reported for some patients, despite overall improvement with early diagnosis and treatment. Aim: The aim of this workshop is to discuss current management and outcomes across Australasia with emphasis on use of carnitine and medical foods, emergency management plans, protein/lysine counting and relaxation of the diet with age. Method: To facilitate discussion, we will present a review of the literature including updates from the presentations at the Genetic Metabolic Dietitians International Conference held in the United States April 2018 and a U.S. perspective of management and issues by Dr. Rani Singh. We plan to conduct a survey of the current practices in Australasian clinics and these results will be presented and discussed. Conclusion: From this workshop it is hoped that participants will feel more confident in the nutritional management of this complex condition. Acknowledging there are different approaches to nutritional management, it is also hoped the outcome of this workshop could also be the agreement of some general principles, (for example energy calculations and data used for protein/lysine counting), which will guide local management protocols resulting in a consistent approach Australasia wide.

\section{Special Interest Group Meetings \\ 4 August 2018 \\ Australasian Society of Diagnostic Genomics ISOLATION OF CIRCULATING FETAL TROPHOBLASTS USING INERTIAL MICROFLUIDICS COMBINED WITH SINGLE CELL ANALYSIS \\ Rezaei $M^{\prime}$, Winter $M^{\prime}$, Hardy $T^{2}$, Zander-Fox $D^{3}$, Warkiani $M^{4}$, Thierry $B^{\prime}$ \\ ${ }^{1}$ Future Industries Institute and ARC Center of Excellence for Convergent Bio-Nano \\ Science,University of South Australia, Adelaide, Australia \\ ${ }^{2}$ Women's and Children's Hospital, Adelaide, Australia \\ ${ }^{3}$ Monash IVF Group, Melbourne, Australia \\ ${ }^{4}$ University of Technology Sydney, Sydney, Australia}

Background: Cell-free fetal DNA (cffDNA) non-invasive prenatal testing (NIPT) has gained broad clinical acceptance for the detection of a number of common genetic disorders. However, cffDNA NIPT cannot currently provide information about the full range of genetic abnormalities. As fetal cells have an intact and complete fetal genome, isolation of these cells from maternal blood as a source of fetal genomic DNA for prenatal diagnosis is a tantalizing goal. Aims: Validate the use of inertial microfluidics for the enrichment of fetal trophoblastic cells from maternal blood. We also aim to implement a subsequent reliable methodology for single cell isolation and downstream use for genetic analysis. Method:
As a model of fetal trophoblastic cells we spiked 100 JEG3 cells (trophoblastic-like cell-line) into $8 \mathrm{ml}$ of healthy blood. RBCs were lysed and then JEG3 cells were enriched via inertial microfluidics and stained with anti-CD45/anti-PanCytokeratin/DAPI. The enriched fraction was scanned and enumerated with automated fluorescence microscopy. To demonstrate downstream genetics applicability, single trophoblastic cells were picked up and then sequenced by NGS (MiSeq). Results: We achieved a 75\% recovery of JEG3 cells and a $99.5 \%$ white blood cell depletion. This study demonstrates isolation of JEG3 cells and subsequent single cell sequencing. Subsequently, we demonstrate inertial microfluidic separation of circulating fetal cells from a maternal blood sample. Discussion: With this feasibility study we have demonstrated that inertial microfluidics is a simple, efficient and gentle methodology to enrich trophoblastic cells from maternal blood and that it can be readily combined with downstream single-cell genetic analysis.

\section{DIGITAL KARYOMAPPING PROVIDES A SUPERIOR APPROACH TO PREIMPLANTATION GENETIC DIAGNOSIS FOR COUPLES UNDERGOING GENOMIC AUTOPSY}

Hardy $T^{1,2}$, Byrne $A^{4}$, Lawrence $D^{1,4}$, Arts $P^{4}$, Babic $M^{4}$, King-Smith $S^{4}$, Ledger $\mathrm{W}^{5}$, Scott $\mathrm{H}^{1,4}$, Barnett $\mathrm{C}^{3}$

${ }^{1}$ SA Pathology, Adelaide, Australia

${ }^{2}$ Repromed, Dulwich, Australia

${ }^{3}$ SA Clinical Genetics Service, Women's and Children's Hospital, North Adelaide, Australia

${ }^{4}$ Centre for Cancer Biology, University of South Australia, Adelaide, Australia ${ }^{5}$ University of New South Wales, Sydney, Australia

Background: Whole genome sequencing (WGS) is becoming a standard diagnostic approach in cases of fetal abnormality. Future reproductive planning for these couples may involve preimplantation genetic diagnosis (PGD); however, patients undergoing SNP arraybased karyomapping require validation studies adding further time and expense prior to commencing IVF/PGD. Aim: To generate a digital karyomap of informative variants using WGS trios for use in future IVF/PGD cycles. Methods: Variant call format files for two families undergoing WGS were analyzed according to the principles of karyomapping. Informative variants within a standard karyomapping window of $2 \mathrm{Mb}$ of the gene of interest and intragenic variants were analyzed to confirm correct assignment of pathogenic variants. Results: Two families underwent WGS trios with a molecular diagnosis of compound heterozygous mutations in MDFIC (Family 1) and MKS1 (Family 2). Overall, there were 5,574,901 variants analyzed in Family 1 of which 3,107,644 were informative (55.74\%). Family 2 had 5,720,198 variants of which 3,389,623 were informative $(59.26 \%)$. There were 2,837 variants within a $2 \mathrm{Mb}$ window of MDFIC and 3,597 variants within a 2Mb window of MKS1. Pathogenic variants were correctly assigned within MDFIC and MKS1. SNP array would have detected $2.22 \% / 2.06 \%$ of informative variants. Conclusion: WGS trio data can be used to generate a digital karyomap that provides a much higher resolution and therefore more informative assessment of the gene of interest, in a much shorter time frame, than current techniques. Couples will therefore be able to proceed straight from genomic diagnosis to IVF with PGD, improving transition to reproductive services.

PRENATAL DIAGNOSIS FOR SINGLE GENE DISORDERS IN VICTORIA, AUSTRALIA, 1977-2015

Poulton $\mathrm{A}^{\mathrm{I}, 4}$, Lewis $\mathrm{S}^{1,4}$, Hui $\mathrm{L}^{1,2,3}$, Halliday $\mathrm{J}^{1,4}$

${ }^{1}$ Public Health Genetics Group, Murdoch Children's Research Institute, Parkville, Australia

${ }^{2}$ Department of Obstetrics and Gynaecology, University of Melbourne, Heidelberg, Australia

${ }^{3}$ Department of Perinatal Medicine, Mercy Hospital for Women, Heidelberg, Australia

${ }^{4}$ Department of Paediatrics, University of Melbourne, Parkville, Australia

Background: Since genetic prenatal diagnosis (PNDx) became available in the 1970s, significant advances have occurred in our 
knowledge of the human genome. More recently, preimplantation genetic diagnosis (PGD) has provided additional reproductive options for couples. Aim: To examine the state-wide utilization of PNDx and PGD for single gene disorders since the clinical introduction of these technologies. Methods: Population-based study of all Victorian women utilizing PNDx from 1977 to 2015. Single gene disorders were categorized using a systematic approach that aimed to reflect aspects of the PNDx decision-making process. Data on PGD for single gene disorders from 2005 to 2016 were similarly examined for comparison. Statistical significance testing was performed with chi squared test for trend. Results: Following an initial uptake period of PNDx for single gene disorders, testing numbers steadied at $\sim 115$ p.a since the late 1990 s. The annual range of disorders doubled from 22 in 1993 to 45 in 2015. Most PNDx (68\%) was performed for disorders that primarily impair physical ability, while PNDx for adult onset conditions (3\%) was low. PGD for single gene disorders has seen rapid growth, and now equals the numbers of PNDx. In contrast to the PNDx experience, 25\% of PGD tests are performed for adult onset conditions. Conclusions: Over the past four decades, both the annual number and scope of reproductive testing for single gene disorders has grown considerably. PGD has emerged as an equal contributor with PNDx for the identification of affected conceptions, but is the preferred diagnostic option for adult onset disorders.

\section{RETROSPECTIVE ANALYSIS SUGGESTS CLINICAL OUTCOMES ARE DIFFERENT FOR WHOLE CHROMOSOME, SEGMENTAL AND COMPLEX MOSAIC EMBRYOS.}

\section{Everett $A^{\prime}$, Grkovic $S^{\prime}$, Stockton J', McArthur $S^{\prime}$}

${ }^{1}$ Genea, Sydney, Australia

Background: The use of next generation sequencing (NGS) technology for embryo aneuploidy testing has resulted in much higher detection rates of chromosome mosaicism, compared to microarray. This has necessitated the introduction of a system for ranking the suitability of mosaic embryos for clinical use. Aim: To determine the clinical outcomes following transfer of mosaic embryos separated into three groups: whole chromosome, segmental, or complex ( $\geq 2$ abnormalities). Methods: 47 mosaic embryos were transferred. Biochemical pregnancy (bHCG), fetal heart (FH) and where possible live birth rates were compared between the groups and also to non-mosaic (NAD) embryos. We also assessed the outcomes for mosaicism levels of $20 \%-\leq 40 \%$ versus $>40-<80 \%$. Results: Single segmental mosaic embryos had higher positive bHCG $(57.1 \%)$ and $\mathrm{FH}(50 \%)$ rates compared to single whole chromosome mosaics $(38.1 \%$ bHCG and $28.6 \% \mathrm{FH})$. Single mosaics had clearly better outcomes compared to complex mosaics (bHCG 45.7\%, FH $37.1 \%$ vs bHCG $33.3 \%$, FH $16.7 \%$ ). Although no ongoing pregnancies were achieved for whole chromosome mosaics $>40 \%$, the level of mosaicism did not appear to impact segmental outcomes. Despite these trends, no statistical significance between the above groups was observed due to low sample numbers. However, the FH rates for single chromosome and complex mosaics were significantly worse than NAD embryos, whereas segmental mosaics were not. Conclusion: In the absence of NAD embryos, our data indicates segmental mosaics should be preferentially transferred ahead of whole chromosome mosaics, then complex mosaics.

\section{OPTIMISING VARIANT ANALYSIS FOR FETAL GENOMIC}

\section{AUTOPSY}

Arts $P^{\prime}$, Byrne $A^{\prime}$, Lawrence $D^{2}$, Weisburd $B^{3}$, Babic $M^{\prime}$, Feng $J^{2}$, O'Donnell $A^{3}$, Pais $L^{3}$, Wang $P^{2}$, Lovgren $A^{3}$, Hardy $T^{1}$, King-Smith $S^{1}$, Barnett $C^{4,5}$, Scott $H^{1,2,4}$

${ }^{1}$ Department of Genetics and Molecular Pathology, Centre for Cancer Biology, a SA Pathology \& UniSA Alliance, Adelaide, Australia

${ }^{2}$ ACRF Cancer Genomics Facility Centre for Cancer Biology, SA Pathology, Adelaide, Australia

${ }^{3}$ The Broad institute of MIT and Harvard, Boston, USA

${ }^{4}$ School of Medicine, University of Adelaide, Adelaide, Australia

${ }^{5}$ South Australian Clinical Genetics Service, SA pathology, Women's and Children's hospital, Adelaide, Australia

Loss of a pregnancy or neonate has a major impact on families in Australia and overseas. Despite pathological examination and genetic tests as part of standard care, $\sim 25 \%$ of cases are currently unexplained. Understanding the (genetic) cause of perinatal death is essential to council these families for future pregnancies. Different variant analysis tools used with WES and WGS data may lead to causative mutations being ignored, or to an overwhelming amount of false positive variants. We systematically tested the Seqr (Broad institute) and VariantGrid (in-house) tools to optimize data analysis and variant (SNV) interpretation of WES and WGS data for fetal genomic autopsy on 40 trios. Overall the analysis of 40 trios resulted in identification of (likely) pathogenic mutations in known, genes in $30 \%$ and candidate variants for functional follow up for another $30 \%$. Using similar filters and annotation settings, causative mutations for one case would have been missed using Seqr only, and for another case using VariantGrid only. One de novo mutation in a third case did not pass the quality filter of both analysis tools. We conclude that neither of the tested variant analysis tools is perfect, mostly because the current versions do not include systematic CNV analysis of exome data. The increased 'diagnostic' yield in cases with congenital abnormalities on ultrasound confirms this method for fetal detection of well-studied developmental disorders. The limited current knowledge about the genetic factors involved in perinatal death provide opportunities for novel disease gene discovery in this unique cohort.

\section{PMS2: DIAGNOSTIC TESTING IN THE ERA OF MASSIVELY PARALLEL SEQUENCING - A WESTERN AUSTRALIA PERSPECTIVE}

Buzzacott $N^{\prime}$, Edwards $C^{\prime}$, Ricciardi J', Fietz $M^{\prime}$, Carpenter K'

${ }^{I}$ Diagnostic Genomics, PathWest Laboratory Medicine, QEII Medical Centre, Nedlands, Australia

Lynch syndrome, a heritable form of colorectal, endometrial, stomach and other cancers, is largely characterized by autosomal dominant pathogenic variants in one of four mismatch repair genes MLH1, MSH2, MSH6, and PMS2. Of these genes, variants in PMS2 account for less than $5 \%$ of cases but remain one of the most challenging to investigate due to the presence of at least 15 pseudogenes that display high homology particularly of the last 4 exons. Prior to 2015 PathWest Diagnostic Genomics (formerly Princess Margaret Hospital Genetics) investigated PMS2 using a modified long range polymerase chain reaction (LRPCR) method (Vaughn et al 2010). Since 2015 PMS2 has been investigated via LRPCR in conjunction with massively parallel sequencing (MPS), using the Illumina TruSight Cancer Panel and MiSeq platform. This period of overlap has allowed the contrast of these two methods in approximately 100 patients. Problems inherent with variant calling by MPS, such as the inability to consistently identify insertion/deletion events, were encountered but surprisingly a number of issues relating to the LRPCR method were also identified. Furthermore, the data suggests variant identification by MPS remains problematic in the last 4 exons of the PMS2 gene. This review suggests that a testing strategy including both MPS and LRPCR, supplemented with MLPA for large 
deletions and duplications, provides the most comprehensive variant detection platform for the PMS2 gene.

\section{DETERMINING AGG INTERRUPTIONS IN FMR1 REPEAT REGION FOR RISK ESTIMATION FOR FRAGILE $X$ PREMUTATION CARRIERS}

$\underline{\text { Ho G }}^{1,2}$, Gennery $C^{1}$, Calma J', Bennetts $B^{1,2}$

${ }^{I}$ Sydney Genome Diagnostics, Western Sydney Genetics Program, Children's Hospital Westmead, Westmead, Australia

${ }^{2}$ Discipline of Child \& Adolescent Health; Discipline of Genetic Medicine, University of Sydney, Sydney, Australia

Background: Fragile X syndrome (FXS) is primarily due to instability of a CGG trinucleotide repeat region in the 5' untranslated region of the FMR1 gene, which impacts upon the expression of the gene product. Premutation alleles (55 to 200 repeats) can expand to a full mutation ( $>200$ repeats) upon a single maternal transmission, but the risk of expansion is mitigated by the presence of AGG interrupts within the repeat region. This is particularly relevant for alleles with up to 90 repeats. Previous methods for determining AGG interrupt status gave results that were difficult to interpret in females due to the presence of two X-chromosomes. Aim: To investigate the suitability of different methods for accurate determination of AGG interrupts in FMR1 repeat region in a diagnostic setting. Method/Results: Patients at risk of allele expansion were referred and tested. No singular method was capable of fully resolving ambiguity. The addition of a CGG repeat-primer to AmplideX PCR/CE FMR1 kit (Asuragen Inc, Austin, TX), along with a separate PCR assay using primers specific for the AGG interrupt, combined to give unambiguous data on the number and position of AGG interrupts, including in one patient who was potentially mosaic for an AGG interrupt. Conclusion: By the use of two complementary methods, it is possible to easily and unambiguously determine the number and position of AGG interrupts. Adopting these methods into standard diagnostic fragile $\mathrm{X}$ testing would assist in clarifying the risks in carriers for premutations and better enable them to make informed reproductive decisions.

\section{SPLICING VARIANT IN A NON-CANONICAL ISOFORM OF TTN IS ASSOCIATED WITH ARTHROGRYPOSIS IN FOUR FAMILIES}

Bryen $S^{1,2}$, Pinner $\rfloor^{3}$, Ewans $L^{3}, O^{\prime} G r a d y ~ G^{1,2}$, MacLennan $S^{8,9}$, Donkervoort $S^{4}$, Cummings $B^{5,6}$, Chao $K^{5,6}$, Weisburd $B^{5,6}$, Bonnemann $C^{4}$, MacArthur $D^{5,6}$, Davis $\mathrm{M}^{7}$, Cooper $\mathrm{S}^{1,2}$

${ }^{I}$ Institute for Neuroscience and Muscle Research, The Children's Hospital at Westmead, Sydney, Australia

${ }^{2}$ Discipline of Paediatrics and Child Health, University of Sydney, Sydney, Australia

${ }^{3}$ Department of Molecular and Clinical Genetics, Royal Prince Alfred Hospital, Sydney, Australia

${ }^{4}$ Neurogenetics Branch, National Institute of Neurological Disorders and Stroke,

National Institutes of Health, Bethesda, USA

${ }^{5}$ Analytic and Translational Genetics Unit, Massachusetts General Hospital, Boston, USA

${ }^{6}$ Medical and Population Genetics, Broad Institute of Massachusetts Institute of Technology and Harvard, Cambridge, USA

${ }^{7}$ Department of Diagnostic Genomics, PathWest Laboratory Medicine, Perth, Australia ${ }^{8}$ Neurology Department, Women's \& Children's Hospital, Adelaide, Australia ${ }^{9}$ Department of Paediatrics and Reproductive Health, Adelaide Medical School, University of Adelaide, Adelaide, Australia

We describe four families presenting with arthrogryposis and congenital myopathy bearing a novel extended splice site variant in intron 212 of TTN c.39974-11T $>$ G within a shared haplotype, inherited in trans with a second pathogenic TTN variant. Phenotypic presentations ranged from arthrogryposis multiplex with pregnancy termination at 26 weeks, to distal arthrogryposis with congenital myopathy. Importantly, contractures presenting at birth resolved for two individuals, with one individual progressing to walk independently aged 6 years. RT-PCR of RNA extracted from skeletal muscle from two patients shows abnormal splicing resulting from the c.39974-11T > G variant; including in-frame skipping of exon 213, or use of a cryptic splice site effecting a frameshift. Confounding interpretation of pathogenicity is the absence of exons 212-216 from the presumed skeletal muscle TTN isoform (NM_133378.4). However, RNA sequencing data from 365 GTEx skeletal muscle control samples reveals; $59 \%$ of individuals predominantly include exons 212-216 in TTN transcripts, $19 \%$ of individuals show predominant skipping of exons 212-216, and $22 \%$ show a mix of both events. Exons 212-216 encode part of the elastic proline-glutamine-valinelysine (PEVK) region of TTN. Previous murine studies show that exons encoding the PEVK region are alternately-spliced, and more frequently included in TTN transcripts in developmental/neonatal muscle, compared to adult muscle (1). Thus TTN exons 212-216 may be important for early development. Collective data therefore provides compelling evidence for likely pathogenicity of the recurrent TTN c.39974-11T > G splice variant. Our results extend emerging evidence showing TTN variants may be associated with severe, arthrogryposis multiplex, or congenital myopathy with distal arthrogryposis.

\section{TOWARDS THE DEVELOPMENT OF A SCREENING ALGORITHM FOR HEMOGLOBINOPATHIES IN AUSTRALIA}

Cliffe $\mathrm{C}^{1}, \mathrm{Ai} \mathrm{S}^{2,3}$, Kidson-Gerber $\mathrm{G}^{2,3}$

${ }^{I}$ NSW Health Pathology Molecular Genetics, Randwick, Australia

${ }^{2}$ NSW Health Pathology Haematology, Randwick, Australia

${ }^{3}$ University of NSW, Kensington, Australia

The ethnic diversity of the Australian population hampers the development of standardized screening for hemoglobinopathies. An audit of molecular genetic hemoglobinopathy investigations by NSWHP Genetics and Haematology departments between January 1, 2015 and December 31, 2017, was performed to inform clinical and laboratory practice and develop screening algorithms for this region. Genetics results were correlated with results of full blood count, mean cell volume (MCV), mean cell hemoglobin $(\mathrm{MCH})$ and hemoglobin electrophoresis. In the 400 individuals referred for HBA analysis, 211 HBA variant alleles were observed, with -a3.7 (56\%) and -SEA (26\%) variants observed most frequently. Mean MCV for HBA deletions of 3 genes was 62.2fl (range 50.4-75.6 fl), 2 genes in cis was $67.2 \mathrm{fl}$ (range 58.1-81.6 fl), 2 genes in trans was 70.6fl (46.7-76.0 fl) and single genes was $76.3 \mathrm{fl}$ (range $58.0-86.0 \mathrm{fl}$ ). In the $143 \mathrm{in}-$ dividuals with HBB analysis, 99 variants were detected, comprising 27 unique variants, with $\mathrm{HbS}$ accounting for $29 \%$. The average $\mathrm{HbA} 2$ for individuals with beta plus variants was $4.5 \%$ (range 2.4 $6 \%$ ) and beta zero variants $5.1 \%$ (range $2.4-6.3 \%$ ). $\mathrm{HbA} 2$ results were observed outside of the expected range for $10 \mathrm{HBB}$ variants (2.4-3.8\%) and 5 sequence-variant negative (3.4-3.7\%).The high frequency of HBA variants with Middle Eastern (-a3.7) and SouthEast Asian (-a3.7,-SEA) origins reflects Australia's genetic diversity. This data suggests the MCV threshold may be lowered to $<76 \mathrm{fl}$ to capture $2 \mathrm{HBA}$ gene deletions and molecular testing is required to distinguish cis from trans mutations. Silent beta thalassemia requires molecular testing to diagnose with few indicators from screening tests.

\section{ANALYSIS OF BRCA1/2 RELATED BREAST CANCER GENES IN MUTATION-NEGATIVE INDIVIDUALS: A SOUTH AUSTRALIAN PERSPECTIVE}
Thompson-Peach $\mathrm{C}^{1}$, Michael $\mathrm{M}^{2}$, Grist $\mathrm{S}^{3}$, Kuss $\mathrm{B}^{1,3}$, Lower $\mathrm{K}^{1}$
${ }^{1}$ Department of Molecular Medicine and Pathology, Flinders University, Bedford Park, Australia
${ }^{2}$ Flinders Centre for Innovation in Cancer, Flinders Medical Centre, Bedford Park, Australia
${ }^{3}$ Department of Molecular Pathology, SA Pathology, Bedford Park, Australia

Breast cancer is the most common cancer affecting Australian women, with many individuals having a strong family history of the disease. While inherited mutations in BRCA1, BRCA2, and 
additional susceptibility genes account for $\sim 30 \%$ of familial breast cancer cases, the underlying cause in the remaining $70 \%$ is unknown, suggesting that additional breast cancer susceptibility genes exist. We hypothesized that mutations within genes that play a role in the DNA damage repair and checkpoint control pathways may be involved in predisposing families to inherited breast cancer. In order to test our hypothesis, Ion Torrent Massively Parallel Sequencing and a custom targeted panel were used to sequence 51 genes of interest in 132 BRCA1/2 mutation-negative individuals with familial breast cancer. The gene panel consisted of 19 known breast cancer susceptibility genes (diagnostic genes) and 32 genes which play integral roles in the aforementioned pathways and therefore are potentially involved in the development of breast cancer (discovery genes). Sequencing analysis identified $\sim 120$ variants in each sample, with variants present in $<5 \%$ of the population analyzed further. Of the 173 variants which were predicted to alter gene transcription or translation, 71 variants (42 diagnostic; 29 discovery) were identified as being potentially pathogenic. A pathogenic truncation mutation was identified in PALB2 in 2 individuals. CRISPR/Cas9 was used to functionally validate a UIMC1 polymorphism identified in 2 patients. This research has the potential to provide much needed diagnostic information for the identification of mutations resulting in familial breast cancer, and to identify novel breast cancer genes.

\section{Australasian Society of Inborn Errors of Metabolism} NATIONAL NEWBORN SCREENING IN AUSTRALIA

\section{Wiley $\mathrm{V}^{1,2}$ \\ ${ }^{1}$ NSW Newborn Screening Programme, Westmead, Australia \\ ${ }^{2}$ University of Sydney, Sydney, Australia}

Newborn screening began in Australia in 1964 with screening for phenylketonuria in one state (NSW). Today newborn blood spot screening is offered to all babies born (approximately 330,000 births per year) by one of 5 publically funded state based programs. One state (Tas) and 2 territories (ACT and NT) with births of less than 10,000 each per year contract for testing in one of the existing programs. While guidelines for newborn screening practice are offered by professional societies (HGSA and ACP) it is up to each state program to decide what is included and therefore the screening pathway and disorders screened for a baby depends on where it is born. In order to provide equity of service and set a clear pathway for deciding what should be included in the future there has been a national initiative under the Standing Committee on Screening (SCOS) to develop a policy framework. A Newborn Bloodspot Screening Working Group (NBSWG) was formed in 2013 with representatives from state and federal governments, professionals from screening for program and laboratory aspects, other health professionals, a bioethicist as well as a consumer representative. The NBSWG has developed a policy, which includes program overview; program implementation; quality and safety; monitoring, evaluation and review; and decisionmaking framework. This policy built on the strengths and successes of the last 50 years of screening in Australia will provide clear policy guidance, support consistency, with increased transparency as well as enable structured assessment of disorders for addition or removal.
NEWBORN SCREENING RESULTS OF INFANTS BORN TO MOTHERS WITH DIABETES IN PREGNANCY TREATED WITH METFORMIN.

Estrella $\mathrm{J}^{1,2}$, Simmons $\mathrm{D}^{1,2}$, McLean $\mathrm{M}^{3,4}$, Hng $\mathrm{T}^{3,4}$, Lau $\mathrm{S}^{3,4}$, Wiley $\mathrm{V}^{5,6}$

${ }^{I}$ Western Sydney University School of Medicine, Macarthur Clinical Campus, Australia ${ }^{2}$ Macarthur Diabetes and Endocrine Services, Campbelltown and Camden Hospitals, Australia

${ }^{3}$ Western Sydney University School of Medicine, Blacktown Clinical School, Australia ${ }^{4}$ Department of Diabetes and Endocrinology, Blacktown-Mt Druitt Hospitals, Australia ${ }^{5}$ NSW Newborn Screening Program, Childrens Hospital at Westmead, Australia ${ }^{6}$ University of Sydney, Discipline of Pediatrics and Child Health and Discipline of Genetic Medicine, Sydney, Australia

Background: Diabetes in pregnancy (DIP: type 1, type 2 and gestational diabetes) inadequately controlled by lifestyle, has traditionally been treated with insulin, as insulin does not cross the placenta, is safe and effective. Metformin is a biguanide analogue which inhibits liver mitochondrial glycerophosphate dehydrogenase and decreases gluconeogenesis. It is effective in the treatment of gestational diabetes and has the advantage of oral administration. Use of metformin in patients with type 2 diabetes has been shown to decrease levels of phenylalanine (phe) and tyrosine (tyr) 1 , amino acids associated with diabetes development. However, metformin crosses the placenta and some studies have shown associations with increased body fat in infants exposed to metformin. We aimed to examine newborn screening (NBS) results of infants born to mothers with DIP treated with metformin. Methods: Consent was obtained for retrospective evaluation of the NBS results of 498 pregnancies (delivered between 2007-2014) involving metformin-treated mothers. Exclusions included prematurity, critical illness and other potential confounders. Amino acids were quantitated using electrospray ionization tandem mass spectrometry. Results were compared per year against the pooled population results of 100,000 newborns per year. Results: 416 NBS results were included. There was skewing of phe and tyr above the median for each year in newborns exposed to metformin antenatally. Conclusion: Metformin use during pregnancy shows a detectable difference in NBS results, providing some evidence of an effect on neonatal liver metabolism. Whether this is due to diabetes exposure or metformin, and its clinical relevance, needs further follow-up.

ANALYSIS OF THYROID PEROXIDASE GENE MUTATIONS IN PATIENTS WITH CONGENITAL THYROID DYSHORMONOGENESIS

Begum $M^{1,2}$, Islam $M^{2}$, Sarker $S^{1,2}$, Bhuyan $G^{2}$, Sajib $A^{\prime}$, Islam $A^{\prime}$ Akhteruzzaman SI, Qadri S², Qadri F $F^{2,3}$, Mannor $\mathrm{K}^{2}$

${ }^{I}$ Department of Genetic Engineering and Biotechnology, University of Dhaka, Dhaka, Bangladesh

${ }^{2}$ Laboratory of Genetics and Genomics, Institute for Developing Science and Health Initiatives, Mohakhali, Dhaka, Bangladesh

${ }^{3}$ Department of Enteric and Respiratory Infectious Diseases, Infectious Diseases Division, icddr,b, Mohakhali, Dhaka, Bangladesh

Background: Congenital hypothyroidism $(\mathrm{CH})$ is a condition of inadequate production of thyroid hormones resulting from an absent or under-developed thyroid gland (dysgenesis) or defect in the synthesis (dyshormonogenesis) of the thyroid hormones that affects infants from birth. The frequency is 1 in 1,300 children in Bangladesh and there is limited data regarding genetic etiology of congenital hypothyroidism. Thyroid peroxidase (TPO) is the major gene involved in dyshormonogenesis. Aims: In this study, we wanted to correlate the effect of specific mutations in TPO gene with clinical features as well as in the 3D structure of the protein. Methods: A total of 36 confirmed cases of congenital hypothyroid children were enrolled in the clinical settings of BSMMU. Three $\mathrm{ml}$ of blood specimen was collected for genomic DNA isolation and amplification of TPO gene (Exon 8 to Exon 14) by PCR followed by Sanger Sequencing. Bioinformatics analysis was performed to explore the specific mutations and its effects in 3D structure of the protein. Results: Thirteen out of 
36 patients had mutation(s) in Exon 8, 6 patients had mutation(s) in Exon 12, whereas 17 patients had mutation in both Exon 8 and Exon 12 of TPO gene. Single nucleotide changes such as c.1117G $>$ T (Ala373Ser), c.1193G $>$ C (Ser398Thr), c.2145C $>$ T (Pro715Pro) and c.2173A $>C$ (Thr725Pro) were found. Among them two of the amino acids regarding mutations were residues of catalytic site of the TPO enzyme. Conclusion: The correlation between mutation and functional activity will help to better understand the disease severity in hypothyroid patients.

\section{THE COMPARISON OF 2 FOURPLEX QUANTITATIVE PCR} ASSAYS FOR TREC, KREC, SMN1 AND RNAseP

\author{
Wotton $T^{1}, K i m W^{1}$, Theresa $S^{1}$, Hjort $M^{3}$, Payne $M^{4}$, Wiley $V^{1,2}$ \\ ${ }^{I}$ NSW Newborn Screening Programme, Westmead, Australia \\ ${ }^{2}$ University of Sydney, Sydney, Australia \\ ${ }^{3}$ Perkin Elmer, Turku, Finland \\ ${ }^{4}$ Thermo Fisher Scientific, North Ryde, Australia
}

DNA multiplex technology has the potential to change the range of conditions currently screened for using the newborn screening blood spot. For example, using T-cell receptor excision circle (TREC) and kappa-deleting recombination excision circle (KREC) as markers for primary immunodeficiencies and analysis of survival of motor neuron 1 (SMN1) mutations associated with spinal muscular atrophy (SMA). The feasibility and reliability of two four-plex commercial quantitative PCR assays, which directly measure the copy numbers of SMN1, TREC, KREC and a reference gene (RNAseP), were assessed on two different analytical platforms, the ThermoFisher QuantStudio and the Roche LC480. Evaluation of the assays was undertaken with 2000 de-identified prospective randomly selected DBS samples which had consent for research, 20 positive controls for each marker, sex and matrix matched negative controls samples (two per positive control). DNA was extracted from all samples using an automated liquid handler and all samples were successfully amplified. Of the 2000 newborn samples none indicated absence of SMN1 or low TREC or KREC numbers. There was complete concordance of results between the two four-plex quantitative PCR assays. Furthermore, the precision, analytical sensitivity and specificity were assessed and were comparable between the two assays. The turnaround time from receipt of sample to obtaining screening results from both assays was calculated to be less than 1 working day. Hence, both quantitative PCR assays are robust and reliable for newborn screening purposes and upscaling for population screening in the NSW Newborn Screening Programme, over 100,000 births per year, is feasible.

\section{EXPANDING THE USE OF MSMS}

\section{Kevin Carpenter}

Australasian Association of Clinical Biochemists, Alexandria, NSW, Australia

Mass spectrometry has been an important tool in Biochemical Genetics for the past half century. Its use has allowed the discovery of new classes of disorders (organic acids by GC/MS) and allowed the expansion of newborn bloodspot screening to encompass many conditions with a single test. However, the use of mass spectrometry has largely been restricted to the role of a sensitive and specific detector in gas or liquid chromatography systems or in a limited targeted metabolomic role using LC-MSMS. The omics revolution is about to reach the diagnostic laboratory and this will require an expansion of our traditional use of mass spectrometry to include untargeted metabolomics as first line screening tests for inborn errors of metabolism. This will bring its own problems of interpretation, analogous to the variants of uncertain significance (VUS) in genomics, but mass spectrometry can play an important role in clarifying the pathogenicity of VUS through flux analysis using stable isotope labelled substrates. This presentation will review the historical use and future direction of clinical mass spectrometry. It will also include a brief look at emerging technological improvements that may even make the mass spectrometer a point of care instrument.

\section{A NEW METHOD TO IMPROVE THE DETECTION OF PLASMA STEROLS, CHOLESTANOL AND VLCFA}

Cox D', McWhinney $B^{\prime}$, McGill J', Stoodley $S^{\prime}$, Ungerer J'

${ }^{1}$ Pathology Queensland, Brisbane, Australia

Background: Current methods used for the analysis of Fatty Acids, Sterols and Cholestenol in human plasma involve extensive extractions, derivatization and lengthy run times. We have developed two simple methods for measuring plasma Sterols and Cholestanol and VLCFA using Waters Ultra Performance Convergence Chromatography with high sensitivity Mass Spectrometry (UPC ${ }^{2}-\mathrm{MS} / \mathrm{MS}$ ). Methods: Both methods utilize a simplified approach which is a benefit of using $\mathrm{UPC}^{2}$ technology. Plasma/serum and deuterated internal standards are hydrolysed and incubated. Following incubation the samples are centrifuged and supernatant is injected into the UPC ${ }^{2}-$ MS/MS system. For the analysis of Sterols and Cholestanol the analytes are separated using a BEH 2-Ethylpyridine column with a total run time of 7 minutes per sample. VLCFA, Pristanate and Phytanate are separated using a HSS $\mathrm{C}_{18}$ column with a run time of 5 minutes per sample. Results: The linear ranges for each analyte are fit for purpose. For each analyte, the coefficient of variation (\% CV) was $<10 \%(n=20)$ and $<10 \%(n=10)$ for intra- and inter-run respectively. Carryover was insignificant, detection limits are suitable for clinical interpretation and analytes correlated well with current GCMS methods. Conclusion: We have developed two simple UPC ${ }^{2}-$ MS/MS assays that measure and detect 7-Dehydrocholesterol, 8Dehydrocholesterol, Lanosterol, Lathosterol, Desmosterol, Sitosterol, Cholesterol and Cholestanol and VLCFA - C22, C24 and C26, Pristanate and Phytanate in biological samples. Both methods show acceptable precision, carryover and specificity. These new tests increase laboratory efficiency and improved results by utilising $\mathrm{UPC}^{2}$ technology to overcome common chromatographic problems for samples possessing a wide range of polarities.

\section{URINE METABOLIC SCREEN: IMPROVING THE DETECTION} RATE OF INBORN ERRORS OF METABOLISM BY LCMSMS

\section{Moore $\mathrm{F}^{\prime}$ \\ ${ }^{I}$ The Children's Hospital at Westmead, Westmead, Australia}

The urine metabolic screen offers a simple method of analysing metabolic intermediates that accumulate due to deficiencies in one or more metabolic enzymes. Traditionally, in our laboratory, the urine metabolic screen included specific assays for amino acids, organic acids and glycosaminoglycans. Testing for other metabolites required a specific request from the consulting physician. To increase the diagnostic yield, the qualitative amino acid analysis previously performed by high voltage electrophoresis, was replaced by an LCMSMS method that measured amino acids, creatine metabolites and purine and pyrimidines plus other significant metabolites.

Urine samples are diluted to a creatinine of $1 \mathrm{mmol} / \mathrm{L}$ before pipetting into two microtitre plates for positive and negative ion analysis. For positive ion analysis the samples are butylated, dried and further diluted before LCMSMS analysis for 27 metabolites and 16 internal standards. For negative ion analysis the samples are simply diluted in the running phase prior to analysis of 16 metabolites and 3 internal standards. Separation of metabolites by chromatography limited the amount of interferences by other compounds and ion suppression due to the sample matrix. Run times are $<4$ minutes per sample. In the first 12 months since the introduction of this method we have detected 25 patients with inborn errors of metabolism including 8 with disorders we previously would not have detected. The introduction of this method provided a more reliable quantitative method for amino acid analysis and by the inclusion 
of many more metabolites allowed for the detection of additional disorders.

\section{A NEONATAL PRESENTATION OF HSD10 MITOCHONDRIAL DISEASE}

Pitt J ${ }^{1,4}$, Bursle $C^{2}$, Christodoulou $J^{1,3,4}$, Eggington $M^{1}$, Hong $K^{1}$, Lunke $S^{1}$, Marum $J^{1}$, Mishra $A^{\prime}$, Odontiadis J', Prentice $\mathrm{T}^{4,5}$, Kee $\mathrm{P}^{5}$, Peters $\mathrm{H}^{2}$, Stark $\mathrm{Z}^{1,4}$

${ }^{1}$ Victorian Clinical Genetics Services, Melbourne, Australia

${ }^{2}$ Department of Metabolic Medicine, Royal Children's Hospital, Melbourne, Australia ${ }^{3}$ Neurodevelopmental Genomics Research Group, Murdoch Children's Research Institute, Melbourne, Australia

${ }^{4}$ Department of Paediatrics, University of Melbourne, Melbourne, Australia

${ }_{5}^{5}$ Neonatal Medicine, Royal Children's Hospital, Melbourne, Melbourne, Australia

HSD10 disease (OMIM 300438) is caused by mutations in HSD17B10 on the X-chromosome. We describe a male neonate presenting with features of mitochondrial disease on day 1: lactic acidosis (11.8; RR $<1.8 \mathrm{mmol} / \mathrm{L})$, hypoglycemia, encephalopathy and hypertonia. Urine organic acid screening on day 3 showed increases in 3-hydroxy-2-methylbutyric and tiglylglycine with normal 2-methylacetoacetic acid, suggestive of HSD10 disease, against a background of non-specific increases in metabolites often associated with mitochondrial diseases such as ketones, dicarboxylic acids and tyrosine metabolites. Newborn screening showed non-specific increases in alanine, tyrosine and methionine, C5:1 carnitine at the upper end of the range $(0.16 \mu \mathrm{mol} / \mathrm{L} ;>99$ th percentile; cut-off 0.27$)$ and normal OHC5 carnitine $(0.09 \mu \mathrm{mol} / \mathrm{L}$; cut-off 0.7$)$. Trio ultrarapid exome sequencing (time to result: $65 \mathrm{hr}$ ) identified a maternally inherited, previously reported, c.388C $>$ T; p.(Arg130Cys) HSD17B10 mutation. The HSD17B10 protein is a multifunctional mitochondrial protein. Its functions include 3-hydroxy-2methylbutyryl-CoA dehydrogenase activity in the isoleucine pathway, the metabolism of neuroactive steroids and as a sub-unit of mitochondrial ribonuclease $\mathrm{P}$ which is involved in the synthesis of $\mathrm{tR}$ NAs. The latter activity results in multiple mitochondrial abnormalities including abnormal morphology. The patient was commenced on a 'vitamin cocktail' with subsequent gradual neurological improvement. He was extubated, established on regular breast feeds and maintained a clinically stable state. This case emphasizes that newborn screening for HSD10 disease can be non-informative and urine organic acids profiles can be subtle and potentially confounded by secondary metabolites. Rapid exome sequencing was helpful in providing diagnostic confirmation and guiding treatment.

\section{MUTATIONS IN TRAPPC4 ASSOCIATE WITH GLOBAL NEURODEVELOPMENTAL DISORDER CHARACTERIZED BY REGRESSION, MICROCEPHALY AND SPASTIC QUADRIPARESIS}

Van Bergen $\mathrm{N}^{1,2}$, Guo $\mathrm{Y}^{3}$, Massey $\mathrm{S}^{1}$, Ranasinghe $\mathrm{T}^{1}$, Antony $\mathrm{J}^{4}$, Collins $\mathrm{F}^{4,5}$, Wilson $\mathrm{K}^{4}$, Hakonarson $\mathrm{H}^{3}$, Christodoulou J 1,2,4,6,7

${ }^{1}$ Neurodevelopmental Genomics Research Group, Murdoch Children's Research Institute, Parkville, Australia

${ }_{2}^{2}$ Department of Paediatrics, University of Melbourne, Melbourne, Australia

${ }^{3}$ Center for Applied Genomics (CAG), Children's Hospital of Philadelphia,

Philadelphia, USA

${ }^{4}$ Western Sydney Genetics Program, Children's Hospital at Westmead, Sydney, Australia

${ }_{5}^{5}$ Medical Genomics Department, Royal Prince Alfred Hospital, Sydney, Australia

${ }^{6}$ Disciplines of Genetic Medicine and Child and Adolescent Health, University of

Sydney, Sydney, Australia

${ }^{7}$ Victorian Clinical Genetics Services, Royal Children's Hospital, Parkville, Australia

The transport protein particle (TRAPP) complex regulates key intracellular trafficking events between the endoplasmic reticulum and Golgi apparatus, potentially influencing a number of cellular processes including autophagy. TRAPP proteins are highly expressed in the brain and pathogenic variants in specific TRAPP proteins are associated with neurological disorders. We undertook whole exome sequencing of a family with two sibs with early onset seizures, intellectual disability, micro- cephaly, sensorineural deafness, spastic quadriparesis and progressive cortical and cerebellar atrophy. We identified a homozygous mutation in a non-canonical well-conserved splice site within TRAPPC4 (hg19:11:g.118890966A>G; TRAPPC4: NM_016146; c. $454+3 \mathrm{~A}>\mathrm{G}$ ) predicted to cause aberrant splicing. RT-PCR from proband fibroblasts demonstrated both full-length transcript and transcripts missing exon 3, suggesting the variant causes a 'leaky' splicing defect. In keeping with this, TRAPPC4 protein expression was significantly reduced. Native PAGE also demonstrated less fully assembled TRAPP complex. Intracellular trafficking through the Golgi was analyzed using a marker protein (VSVG-GFP-ts045) which accumulates in the ER under non-permissive temperatures $\left(40^{\circ} \mathrm{C}\right)$ then upon temperature reduction $\left(32^{\circ} \mathrm{C}\right)$ moves to the Golgi and is transported to the plasma membrane. Co-localization of VSVG-GFP-ts045 with the cis-golgi marker GM130 demonstrated delayed entry and exit from the Golgi in patient cells. Lentiviral rescue with wildtype TRAPPC4 increased protein expression levels, and restored trafficking through the Golgi in patient cells. Given that TRAPPC4 is highly expressed in neurons, and mutations in other TRAPP complex are associated with neurological disorder, we propose that pathogenic variants of TRAPPC4 are likely to be the cause of these children's neurological disease.

\section{IDENTIFYING VARIANTS ASSOCIATED WITH EXERCISE ADAPTATIONS USING WHOLE MITOCHONDRIAL GENOME SEQUENCING IN THE GeneSMART STUDY}

Harvey $N^{1,2}$, Albury $C^{\prime}$, Benton $M^{1}$, Eccles $D^{4}$, Lee $R^{1}$, Haupt $L^{1}$, Ashton $K^{2}$, Eynon $\mathrm{N}^{3}$, Griffiths $\mathrm{L}^{\prime}$

${ }^{1}$ Bond University, Gold Coast, Australia

${ }^{2}$ Queensland University of Technology, Institute of Health and Biomedical Innovation, Brisbane, Australia

${ }^{3}$ Victoria University, Institute of Sport, Exercise and Active Living, Melbourne, Australia

${ }^{4}$ Victoria University, Malaghan Institute of Medical Research, Wellington, New Zealand

Mitochondria are the 'power-house' of the cell, providing the energy required for activities of daily life. We have previously shown that exercise adaptations in active individuals are strongly influenced by sequence variations in the human genome $(1,2)$. However, it is unclear if and how genetic variations in the whole mitochondrial genome influencing training responses. Aim: to identify mitochondrial variants associated with exercise adaptations in a cohort of moderately trained participants. Methods: The Genes and Skeletal Muscle Adaptive Response to Training (Gene SMART) study consists of 77 participants who complete 4 weeks of High Intensity Interval Training (HIIT) at 80\% VO2Max. Phenotypic and molecular measures were taken pre-and-post HIIT (3). Participants were stratified into high response and low response groups based on collective grouping for all measures. A high throughput method developed at the GRC was then used to sequence the mitochondrial genomes using the Ion Torrent PGM platform. Raw BAM files were aligned to the rCRS reference genome and annotated using online mitochondrial genome analysis tools (Mitomaster, mitosuite, haplogrep2). Results: We identified 17 mitochondrial variants significantly associated with the response to strenuous exercise. Two variants in two separate mitochondrial genes were identified as both coding and leading to non-synonymous amino acid changes. Discussion: our preliminary results suggest that 17 mitochondrial variants are associated with exercise responses. Further analysis is underway to determine if these variants can distinguish between high responders and low-responders to HIIT. 
DIAGNOSIS AND TREATMENT OF TYROSINEMIA TYPE I: A US AND CANADIAN CONSENSUS GROUP REVIEW AND RECOMMENDATIONS

Jeffrey Chinsky'; Rani Singh ${ }^{2}$; Can Ficicioglu ${ }^{3}$; Clara Van Karnebeek ${ }^{4}$; Markus Grompe $^{5}$; Grant Mitchell ${ }^{6}$; Susan Waisbren ${ }^{7}$; Muge Gucsavas-Calikoglu ${ }^{8}$; Melissa Wasserstein ${ }^{9}$; Katie Coakley ${ }^{2}$ C. Ronald Scott ${ }^{10}$

${ }^{1}$ Johns Hopkins University School of Medicine, Baltimore, USA

${ }^{2}$ Emory University School of Medicine, Atlanta, USA

${ }^{3}$ Perelman School of Medicine at the University of Pensylvania, Philadelphia, USA

${ }^{4}$ BC Children's Hospital Research Institute, University of British Columbia,

Vancouver, Canada

${ }_{5}^{5}$ Oregon Health and Science University, Portland, USA

${ }^{6}$ Chu Sainte-Justine and Universite De Montreal, Montreal, Canada

${ }^{7}$ Boston Children's Hospital, Boston, USA

${ }^{8}$ University of North Carolina, Chapel Hill, USA

${ }^{9}$ The Children's Hospital at Montefiore, New York City, USA

${ }^{10}$ University of Washington School of Medicine, Seattle, USA

Tyrosinemia type I (hepatorenal tyrosinemia, HT-1) is an autosomal recessive condition resulting in hepatic failure with comorbidities involving the renal and neurologic systems and long-term risks for hepatocellular carcinoma. An effective medical treatment with 2-[2nitro-4-trifluoromethylbenzoyl]-1,3-cyclohexanedione (NTBC) exists but requires early identification of affected children for optimal long-term results. Newborn screening (NBS) utilizing blood succinylacetone as the NBS marker is superior to observing tyrosine levels as a way of identifying neonates with HT-1. If identified early and treated appropriately, the majority of affected infants can remain asymptomatic. A clinical management scheme is needed for infants with HT-1 identified by NBS or clinical symptoms. To this end, a group of 11 clinical practitioners, including 8 biochemical genetics physicians, 2 metabolic dietitian nutritionists, and a clinical psychologist, from the United States and Canada, with experience in providing care for patients with HT-1, initiated an evidence- and consensus-based process to establish uniform recommendations for identification and treatment of HT-1. Recommendations were developed from a literature review, practitioner management survey, and nominal group process involving two face-to-face meetings. There was strong consensus in favor of NBS for HT-1, using blood succinylacetone as a marker, followed by diagnostic confirmation and early treatment with NTBC and diet. Given the availability of sensitive newborn screening techniques and effective therapy, the outcomes for this multisystemic disorder should be dramatically improved with the use of more consistent approaches advocated in this presentation.

\section{COMBINED D2-/L2-HYDROXYGLUTARIC ACIDURIA: AN INTERMEDIATE PHENOTYPE WITH MYASTHENIA GRAVIS CRISES AND RESPONSE TO CITRATE TREATMENT}

Kalli Demetriou $K^{\prime}$, Teo $T^{\prime}$, Cairns $A^{2,5}$, Waak $M^{2,5}$, Inwood $A^{\prime}$, Lipke $M^{\prime}$, McGill $\mathrm{J}^{1,5}$, Pitt J $\mathrm{J}^{3}$, Pop $\mathrm{A}^{4}$, Salamons $\mathrm{G}^{4}$, Coman $\mathrm{D}^{1,5,6}$

${ }^{I}$ Department of Metabolic Medicine, Lady Cilento Children's Hospital, Brisbane,

Australia
${ }^{2}$ Department of Neuroscience, Lady Cilento Children's Hospital, Brisbane, Australia

${ }^{3}$ Victorian Clinical Genetics Services, Murdoch Children's Research Institute, Melbourne, Australia

${ }^{4}$ Metabolic Laboratory, Department of Clinical Chemistry, Amsterdam Neuroscience,

VU University Medical Center, Metabolic Unit, Amsterdam, The Netherlands

${ }_{5}^{5}$ School of Medicine, University of Queensland, Brisbane, Australia

${ }^{6}$ School of Medicine, Griffith University, Gold Coast, Australia

Background: Combined D2-/L2-hydroxyglutaric aciduria (DL2HGA) (OMIM \#615182) is caused by pathogenic mutations in the SLC25A1 gene which encodes the mitochondrial citrate carrier (CIC). It is usually lethal in the first years of life, although a sibling pair, who manifested with milder congenital myasthenia gravis has been reported. Aims: To describe the clinical, molecu- lar, and biochemical features of a child with an intermediate phenotype and his response to citrate replacement. History, Methods, Results: The patient was diagnosed biochemically at 3 months of age during an acute life-threatening apnoea. At 7 years of age he has significant global developmental delay, with diurnal fatigability in upper and lower limb function and bilateral ptosis, which were more prominent during an intercurrent illness. Supplementation with citrate $(800 \mathrm{mg} / \mathrm{kg} / \mathrm{day})$ commenced at age 5.5 years of age. Prior to this he had 6 acute life-threatening events of central apnoea with respiratory arrests needing aggressive resuscitation, during intercurrent infective illnesses. LC-MSMS demonstrated elevated D-2-HG $81 \mu \mathrm{mol} / \mathrm{mmol}$ creatinine $(\mathrm{RR}<9.3)$ and L-2-HG $53 \mu \mathrm{mol} / \mathrm{mmol}$ creatinine $(\mathrm{RR}<10.8)$. SLC25A1 sequencing identified c.844C > T; p.Arg282Cy and c.605T >C; p.Met202Thr. Discussion/Conclusion: Depletion of cytosolic citrate and accumulation of mitochondrial citrate inside mitochondria play key roles in the pathophysiology of DL-2HGA, CIC mediates efflux of the mitochondrial citrate and isocitrate for cytosolic malate. SLC25A1 knockdown zebrafish demonstrate neuromuscular junction impairment, indicating a key role for CIC in normal neuromuscular presynaptic function. Our patient represents an intermediate phenotype characterized by episodes of myasthenic crisis during intercurrent illness, which have been averted by citrate supplementation.

\section{DIETARY MANAGEMENT OF MALONIC ACIDURA DIAGNOSED IN THE NEWBORN PERIOD - TWO CASES}

\author{
Thompson $S^{1}$, Mitchell $A^{2}$, Stanway $T^{1}$, Balasubramaniam $S^{1,3}$, Alexander $I^{1,3,4}$, \\ Ellaway $\mathrm{C}^{\mathrm{l}, 3}$ \\ ${ }^{I}$ Genetic Metabolic Disorders Service, The Children's Hospital at Westmead, \\ Westmead, Sydney, Australia' \\ ${ }^{2}$ Department of Nutrition and Dietetics, The Children's Hospital at Westmead, \\ Westmead, Sydney, Australia \\ ${ }^{3}$ Disciplines of Child and Adolescent Health and Genetic Medicine, University of \\ Sydney, Sydney, Australia \\ ${ }^{4}$ Gene Therapy Research Unit, Sydney Children's Hospital Network and Children's \\ Medical Research Institute, Sydney, Australia
}

Malonic aciduria is a rare organic aciduria caused by a deficiency of malonyl-CoA decarboxylase, resulting in inhibition of fatty acid oxidation. The condition usually presents in early childhood with a variable phenotype comprising developmental delay, hypotonia, seizures, and cardiomyopathy. Severe metabolic decompensation including hypoglycemia, metabolic acidosis, lactic acidemia, marked lethargy, seizures and potentially cardiac arrhythmias can occur during catabolic stress. We report dietary management of 2 children now aged 1 and 3 years, diagnosed in the newborn period and treated with a low long chain fat diet, with medium chain triglycerides, carnitine supplementation and fasting and unwell guidelines. The older patient was diagnosed clinically following decompensation on day 3; the younger following positive newborn screening. Both patients were commenced on Monogen feeds supplemented with glucose polymer for unwell plans and when intake was reduced. Specific guidelines on appropriate volumes and feeding times were provided to avoid fasting. Introduction to solids education was based on avoidance of foods with $>3 \%$ long chain fat. While growth and weight gain was appropriate in both patients, there were ongoing concerns over feeding difficulties exacerbated by reflux. Gastrostomy inserted at age $7.5 \mathrm{mths}$ and $3 \mathrm{yrs}$, respectively, was used to supplement oral intake with Monogen. Both patients continue to require regular review by speech pathology due to slow progression with eating, although both can manage a range of textures. Development is age appropriate in the younger patient, but delayed in the 3-year-old. Both have normal echocardiograms. 